\title{
Two-dimensional conformal field theory for disordered systems at criticality
}

\author{
Christopher Mudry, Claudio Chamon, and Xiao-Gang Wen \\ Department of Physics, Massachusetts Institute of Technology, 77 Massachusetts Avenue, Cambridge, Massachusetts 02139
}

(August 8, 2018)

\begin{abstract}
Using a Kac-Moody current algebra with $U(1 / 1) \times U(1 / 1)$ graded symmetry, we describe a class of (possibly disordered) critical points in two spatial dimensions. The critical points are labelled by the triplets $\left(l, m, k_{j}\right)$, where $l$ is an odd integer, $m$ is an integer, and $k_{j}$ is real. For most such critical points, we show that there are infinite hierarchies of relevant operators with negative scaling dimensions. To interpret this result, we show that the line of critical points $\left(1,1, k_{j}>0\right)$ is realized by a field theory of massless Dirac fermions in the presence of $U(N)$ vector gauge-like static impurities. Along the disordered critical line $\left(1,1, k_{j}>0\right)$, we find an infinite hierarchy of relevant operators with negative scaling dimensions $\left\{\Delta_{q} \mid q \in \mathbb{N}\right\}$, which are related to the disorder average over the $q$-th moment of the single-particle Green function. Those relevant operators can be induced by non-Gaussian moments of the probability distribution of a mass-like static disorder.
\end{abstract}

71.55Jv,11.40.-q,11.10Gh

\section{INTRODUCTION}

The plateau transition in the integer quantum Hall effect perhaps provides the most compelling experimental evidence in support of the scaling hypothesis in the theory of localization close to the mobility edge. Scaling with temperature, sample size, as well as dynamical scaling have been observed in two-dimensional degenerate electronic systems subject to a strong magnetic field [1]. (How strong the magnetic field must be is controlled by the ratio of the magnetic length to the mean free path of the system.) Assuming that there exists one and only one relevant diverging length scale $\xi$ at the transition, the experimentally observed scaling is consistent with a power law divergence of $\xi$ on the energy separation from the center of the Landau band (the mobility edge) with scaling exponent $\nu_{\exp } \approx \frac{7}{3}$ [1]. The same exponent has also been measured in numerical simulations of the plateau transition for two-dimensional noninteracting electronic system in the presence of a static (quenched) weakly correlated disorder and a strong magnetic field [1]. It is tempting to interpret these experimental and numerical observations as evidences for the existence of a quantum critical point for a disordered non-interacting two-dimensional system at which, as a function of a strong magnetic field, a zero temperature continuous phase transition between two insulating quantum Hall states takes place. We take this point of view in this paper.

The present understanding of the plateau transition from a theoretical point of view is quite unsatisfactory for two basic reasons. First, it has not been possible to construct a field theory describing a second order phase transition governed by only one diverging length scale with scaling exponent $\nu_{\mathrm{ft}}=\frac{7}{3}$. On the one hand, a semi-classical picture based on the continuum model of percolation yields, in the infinite strong magnetic field limit, the critical exponent $\nu_{\mathrm{per}}=\frac{4}{3}[2]$. Although the classical continuum model of percolation does not reproduce the correct scaling exponent for the plateau transition, it inspired Chalker and Coddington to propose a network model incorporating in a simplified way quantum effects such as tunneling and quantum interferences [3]. Numerical studies of the ChalkerCoddington $(\mathrm{CK})$ network model suggest that the physics essential to the understanding of the plateau transition has been incorporated in it, since, among others, they predict the scaling exponent $\nu_{\mathrm{CK}} \approx \frac{7}{3}$ for the localization length in agreement with "first-principle" numerical simulations [3]. On the other hand, motivated by the mapping of the problem of the Anderson metal-insulator transition onto an effective non-linear $\sigma$ model, Pruisken and collaborators proposed a non-linear $\sigma$ model with the addition of a new topological term to describe criticality at the plateau transition 画. This approach has been partially successful in that it predicts the existence of critical points describing transitions between some insulating phases. However, it is not clear whether those critical points correspond to the plateau transition. Moreover, it has not been possible to calculate their critical properties. Further attempts to identify the effective field theory describing the critical properties of the plateau transition have consisted in approximating the replicated (supersymmetric) non-linear $\sigma$ model with topological term by a generalized replicated (supersymmetric) spin chain [5] ( [6]), or in approximating the CK network model by a replicated Hubbard chain [7]. Unfortunately, all these effective field theories have remained quite intractable to analytical methods. The intrinsic difficulty in calculating the critical indices of the plateau transition is that this transition is between two insulating phases. This should be contrasted with the conventional Anderson metal-insulator transition for which critical properties can be calculated perturbatively from the metallic regime [8.9]. All attempts to calculate the critical properties of an effective field theory presumed to describe the plateau transition are of a non-perturbative nature and have thus far failed. 
Second, a highly non-trivial property of the critical field theory describing the plateau transition is that its operator content must be consistent with the observed phenomenon of multifractality [10]. Multifractality, in the context of the plateau transition, means that infinitely many universal exponents, which are not linearly related, are needed to characterize the scaling of the generalized inverse participation ratios at criticality. It reflects the fact that the inverse participation ratio is not a selfaveraging observable, whereas its logarithm is. Duplantier and Ludwig have shown how difficult it is to find from a stable Lagrangian field theory a critical point characterized by an infinite hierarchy of operators related to the phenomenon of multifractality [11. There are some perturbative calculations in $2+\epsilon$ dimensions and asymptotically close to the mobility edge of disorder averaged generalized inverse participation ratios [12 14]. They are interpreted as evidences for multifractality 15]. It would be highly desirable to obtain non-perturbatively a hierarchy of scaling exponents for disorder averaged generalized inverse participation ratios, and to show how they are related to the universal scaling exponents observed numerically from the scaling with system size of the generalized inverse participation ratios for fixed realizations of the disorder.

It was recently shown that a periodic potential can induce a continuous transition between two quantum Hall states or from a quantum Hall state to a Mott insulator in an anyonic system (at zero temperature) subject to a magnetic field 16,17. It is then natural to probe the fate of such a (pure) quantum critical point upon perturbation by some weak disorder. In fact, such a procedure was used by Ludwig in a different context to show the existence of a new random fixed point for the two-dimensional $q$-state Potts model $(q \geq 2)$ with weak quenched bond randomness [18. Ludwig also showed that the random fixed point for the two-dimensional $q$-state Potts model is characterized by an infinite hierarchy of relevant operators implying a complex scaling behavior related to multifractality [19. A convenient model undergoing an integer quantum Hall effect in the absence of disorder was soon thereafter constructed by Ludwig et al. 20. The critical properties of the pure system can be described by one species of massless Dirac fermions in $2+1$ space-time. In the absence of disorder, the mass $m$ of the Dirac fermions plays the role of a relevant coupling inducing, as it changes sign, a quantized jump in the transverse static conductivity. Ludwig at al. then studied the effect of three types of quenched (static) Gaussian randomness on the properties of the pure critical point: (i) an Abelian vector gauge-like impurity potential $A_{\mu}, \mu=1,2,(i i)$ an Abelian scalar-like impurity potential $V$, (iii) and a mass-like impurity potential $M$. The independent strengths of the three impurity potentials are measured by the variances $g_{A, V, M}$ of the white noise probability distributions $\mathcal{P}[A], \mathcal{P}[V]$, and $\mathcal{P}[M]$, respectively. To leading order in the impurities strength, the three randomness represent marginal perturbations to the pure critical point. In the presence of only one type of impurities, it was shown in Ref. [20] that, up to second order, $g_{M(V)}$ is marginally irrelevant (relevant), and that $g_{A}$ is exactly marginal to all orders, thus generating a line of critical points starting at the pure limit of the Dirac fermion model. The line $g_{A}>0, g_{V}=0, g_{M}=0$ of critical points is in fact multicritical since Gaussian randomness in $V$ and $M$ induce relevant perturbations [2]. Another important property along the multicritical line $g_{A}>0, g_{V}=0, g_{M}=0$ is that, after proper infrared and ultraviolet regularizations, the amplitude of the typical (with respect to the randomness) critical wave function in a large box (eigenstate obeying periodic boundary conditions with vanishing energy) is expected to be selfsimilar down to a microscopic length scale (the ultraviolet cutoff) and to scale like a multifractal measure with the system size (the infrared cutoff). Because the CK model can be viewed as a random tight-binding Hamiltonian, there must exist a lattice regularization of a field theory of random massless Dirac fermions describing the plateau transition. Ludwig et al. then conjectured that there is, in the three-dimensional coupling space $\left\{g_{A, V, M}>0\right\}$, a stable fixed point which describes the critical properties of the plateau transition in the integer quantum Hall effect. However, if this fixed point exists, it cannot be reached perturbatively from any point of the multicritical line $g_{A}>0, g_{V}=0, g_{M}=0$.

In this paper, we study models of random massless Dirac fermions in two spatial dimensions. These effective field theories describe, in the presence of static disorder, the physics of the low energy and long wave-length singleparticle excitations of quasi two-dimensional electronic systems which are characterized by isolated Fermi points. Examples for which models of random Dirac fermions are appropriate include degenerate semi-conductors [21], twodimensional graphite sheets [22], tight-binding Hamiltonians in the flux phase [23], and dirty $d$-wave superconductors in two dimensions 24,25]. Models of random massless Dirac fermions are closely related to models on the effect of randomness on criticality in statistical mechanics (e.g., two-dimensional Ising 26,27] and $q$-states random Potts model [18,19]), since, at the energy of interest, the pure Dirac system is already critical. In the pure system and at the critical (vanishing) energy, the spatial decay of Green functions is algebraic. Our goal is threefold. First, the study of two-dimensional models of random Dirac fermions might yield a systematic classification of some new disordered

\footnotetext{
1 This is to be contrasted with gap scaling in usual critical phenomena. An example of gap scaling occurs in the twodimensional Ising model. If the scaling of the susceptibility for the two-dimensional Ising model is controlled by the exponent $\eta$, then the scaling of the $n$-th power of the susceptibility is controlled by the exponent $n \eta$.
} 
critical points. Second, their study might shed light on the field theoretical realization of complex scaling behavior (such as multifractality) not present in the pure limit. Third, it might be possible to infer some unconventional properties of the field theory describing the plateau transition from the properties of the field theory describing the multicritical points $g_{A}>0, g_{V}=0, g_{M}=0$, although, as we will see below, they must differ in an important way.

\section{A. Summary of results}

We will assume only one type of randomness: impurities interacting with $N$ independent species of massless Dirac fermions like $U(N)=U(1) \times S U(N)$ (static) vector gauge fields would do. The $U(1)$ case was studied by Ludwig et al. in relation to the plateau transition [20]. The $S U(N)$ case was studied by Nersesyan et al. in relation to $d$-wave superconductivity [25]. Both groups used the replica trick to treat the disorder. We use instead the graded supersymmetric representation of single-particle Green functions [28] to treat the $U(1) \times S U(N)$ vector gauge-like randomness (see also Ref. [29]). The advantage of the graded supersymmetric representation is that with it the operator content at criticality is much easier to obtain than by using the replica trick. The disadvantage is that it is limited to non-interacting systems. In addition to the Abelian randomness $A_{\mu} \in U(1)$ with Gaussian probability distribution of variance $g_{A}$, the non-Abelian randomness is represented by the static vector gauge field $B_{\mu} \in S U(N)$ with Gaussian probability distribution of variance $g_{B}$. We make the important assumption that both $A_{\mu}$ and $B_{\mu}$ are topologically trivial. For example, in the Abelian case, this means that $A_{\mu}$ is smooth everywhere and thus is not associated with a configuration of magnetic monopoles.

We first prove that the generating function for the disorder average of any given product of $m$ retarded and $n$ advanced single-particle Green functions factorizes into $m+n$ pieces which are each independent of disorder, provided all energy scales in the single-particle Green functions vanish, i.e., at the critical energy. This factorization is unique to vector gauge-like randomness. For example, it already fails for scalar-like $V$ or mass-like $M$ randomness. Because of this factorization, we can relate the operator content of the effective field theory for the averaged single-particle Green function at vanishing energy to the operator contents of averaged products of retarded and advanced singleparticle Green functions at vanishing energies. Moreover, we show that, when all energy scales vanish, averaged products of single-particle Green functions are algebraic functions for arbitrary strength $g_{A}>0$ of the Abelian randomness, and in the limits $g_{B}=0, \infty$ for the strength of the non-Abelian randomness. In other words, the generating functions for averaged products of single-particle Green functions describe field theories at criticality along the lines $g_{A} \geq 0, g_{B}=0, \infty$, whenever all energy scales vanish. It is the unconventional properties of these critical effective field theories that are at the heart of this paper.

For the disordered critical points $g_{A} \geq 0, g_{B}=0, \infty$, we calculate the averaged local density of states asymptotically close to the critical energy. We reproduce the results of Ludwig et al. 20] for the Abelian case, and Nersesyan et al. for the non-Abelian case [25]. It is noteworthy to notice that in the Abelian case, the averaged local density of states can be constant or even diverge for strong enough randomness (compare with [30] and [31]). Since the order parameter for the averaged local density of states breaks a continuous symmetry of the effective field theory (the chiral symmetry), any finite or diverging averaged local density of states at criticality (vanishing energy) signifies the breakdown of the Mermin-Wagner theorem. The physical origins of the breakdown of the Mermin-Wagner theorem are two-fold. On the one hand, the pure system of massless Dirac fermions is critical. As a result there exists a continuous chiral symmetry in the effective field theory for averaged product of single-particle Green functions at vanishing energies [23,21]. On the other hand, the effective field theory for the disordered system is non-unitary, whereas the Mermin-Wagner theorem is proven for unitary field theories with compact symmetries. A power law dependency on energy for the averaged local density of states of disordered Dirac fermions is unusual compared to the generic case of a non-critical averaged single-particle Green functions in the problem of localization at the mobility edge such as, say, the plateau transition [32,33]. Note that there are other examples of diverging averaged local density of states in two-dimensional disordered systems [30,31].

The new result of this work is that we can calculate exactly the dominant scaling dimensions $\Delta_{q}$ of arbitrary integer powers $q$ of the local order parameter corresponding to the averaged local single-particle Green function. They are given by

$$
\Delta_{q}=q-\frac{g_{A}}{\pi} q^{2}-\frac{N-1}{N^{2}}\left(q^{2}+N q\right) .
$$

This result is non-perturbative. It yields a spectrum of exponents which are not linearly related, form a concave set, are always relevant, and can be negative: $\Delta_{q} \approx-q^{2}$ for large $q$. The physical meaning of Eq. (1.1) is that the local single-particle Green function, if considered as a random variable, is not selfaveraging, rather it is log-normal distributed at criticality. Eq. (1.1) was already obtained in Ref. [34 for the Abelian case. 
The existence of an infinite hierarchy of operators which are relevant at criticality calls into question the stability of the disordered critical points $g_{A} \geq 0, g_{B}=0, \infty$. As was pointed out by Ludwig et al. [20], impurities inducing a scalar-like randomness $V$ or a mass-like randomness $M$ with Gaussian probability distributions generate relevant perturbations with scaling exponents $\Delta_{2}$. They then conclude that the points $g_{A} \geq 0, g_{B}=0, \infty$ are multicritical [20]. Our result, however, shows that the points $g_{A} \geq 0, g_{B}=0, \infty$ (excluding the pure limit) are unstable in a very special way, namely that there are infinitely many relevant perturbations $\left\{g_{V, M}^{(n)} \mid n \in \mathbb{N}\right\}$ which are generated by all nonGaussian cumulants of the scalar-like or mass-like probability distributions. (For disorder with Gaussian probability distribution, only the variance, say $g_{A, B, V, M}^{(2)} \equiv g_{A, B, V, M}$, is non-vanishing.) Unless there exists a symmetry of the underlying lattice regularization of the random Dirac fermions which forbids randomness in the scalar-like or masslike potentials, the search for the disordered critical points of random Dirac fermions must take place in an infinite dimensional disorder induced coupling space.

It is instructive to contrast our results on the operator content for the disordered critical points $g_{A} \geq 0, g_{B}=0, \infty$ to the $O(n)$ non-linear $\sigma$ model in $2+\epsilon$ dimensions for which there exists a non-trivial critical point $\left(t^{*}, h^{*}\right)=\left(t_{c}(\epsilon)>0,0\right)$ in a two-dimensional coupling space. Both the temperature $t$ and the magnetic field $h$ are relevant perturbations, and both have to be tuned simultaneously to reach the critical point. The fact that the critical point is unstable with respect to $t$ does not mean that there is no physics associated with the $h$ direction: correlation functions such as the magnetization near the critical point will depend on both $t$ and $h$. In our model of random Dirac fermions, there are infinitely many relevant directions, each additional direction corresponding to a new class of randomness (characterized by the number of non-vanishing cumulants). If one were to compute some correlation function (numerically, for example) close enough to the, say, multicritical line $g_{A}>0, g_{V, M}^{(n)}=0$, the correlation function would depend on the form of the probability distribution for the disorder (all $\left.g_{V / M}^{(n)}\right)$ not just on its strength $\left(g_{V / M}^{(2)}\right)$. The multicritical line of Ludwig et al. 20] is unstable in the sense that it is not possible to find in its vicinity a simple scaling form (i.e., depending on a finite number of universal exponents) for correlation functions. It is interesting to note that the non-trivial critical point $\left(t^{*}, h^{*}\right)$ of the $O(n)$ non-linear $\sigma$ model in $2+\epsilon$ dimensions also has infinitely many relevant operators 35. However, they break the $O(n)$ symmetry and cannot be realized physically by switching on an external magnetic field. Hence, they do not destabilize the critical point $\left(t^{*}, h^{*}\right)$. Finally, the validity of the $2+\epsilon$ expansion itself has been questioned, due to delicate issues of convergence [36]37. In Eq. (1.1), the scaling exponents $\left\{\Delta_{q} \mid q \in \mathbb{N}\right\}$ are obtained non-perturbatively, a highly non-trivial result if one attempts to derive it using the replica trick.

We recall that in the theory of localization, the multifractal analysis consisting in first generating, for a given realization of the disorder, so-called box probabilities from the squared amplitude of a normalized energy eigenstate, and then performing a finite size scaling analysis on their $q$-th moment, yields a hierarchy of scaling exponents $\{\tau(q)=D(q)(q-1) \mid q \in \mathbb{N}\}[10]$. When the $\tau(q)$ 's are not linearly related, the wave function is said to be multifractal. Multifractal wave functions are observed for energies close to the mobility edge in two spatial dimensions. More importantly, the $\tau(q)$ 's are observed to be independent of the realization of disorder [10]. It is then tempting to conjecture that $(i)$ the scaling exponents $\{\tau(q)=D(q)(q-1) \mid q \in \mathbb{N}\}$ are universal, and (ii) can be calculated from some critical effective field theory describing the critical wave function in the thermodynamic limit. It turns out that we can relate the spectrum of scaling exponents $\left\{\Delta_{q} \mid q \in \mathbb{N}\right\}$ for powers of the local order parameter corresponding to the averaged local density of states to scaling of the averaged generalized inverse participation ratios introduced by Wegner [12]. This is so because the critical behavior of averaged products of advanced and retarded single-particle Green functions is controlled by the averaged single-particle Green function. Again random Dirac fermions differ in this respect from the conventional critical behavior at the mobility edge in the theory of localization, since in the latter it is the averaged product of one retarded and one advanced single-particle Green function, in other words the amplitude and not the phase of the single-particle Green function, which controls criticality.

We find that, along the critical line $g_{A}>0, g_{B}=\infty$, the scaling of the averaged generalized inverse participation ratios with respect to the energy separation from the critical energy and asymptotically close to it is controlled by the scaling exponents

$$
\varpi(q)=\frac{D^{*}(q)(q-1)}{z}, \quad D^{*}(q)=2-\left(\frac{g_{A}}{\pi}+\frac{N-1}{N^{2}}\right) q, \quad q \in \mathbb{N},
$$

where $z$ is the dynamical exponent:

$$
z=1+\frac{g_{A}}{\pi}+\frac{N^{2}-1}{N^{2}} .
$$

The non-linear contribution in $q$ to the right-hand-side of Eq. (1.2) comes from $\Delta_{q}$. Consequently, the function $\tau^{*}(q) \equiv z \varpi(q)$, when continued to real $q$, is non-linear, concave and unbounded from below for large $q$. The strict 
concaveness of $\tau^{*}(q)$ reflects the non-selfaveraging nature of the squared amplitude of wave functions with energy close to the critical energy. The unboundness of $\tau^{*}(q)$ arises because wave functions have not been normalized. Rare events, i.e., realizations of the disorder such that amplitudes of the wave functions show "giant" spikes, can then dominate the averaging procedure for large $q$.

We would like to stress that the function $\tau^{*}(q)$ only equals $\tau(q)$ for $q=0$ and $q=1$. It is thus too naive to use the sole information contained in the hierarchy of local operators with scaling dimensions $\left\{\Delta_{q} \mid q \in \mathbb{N}\right\}$ (see Eq. (1.1)) to calculate $\tau(q)$ from the critical field theory. Moreover, it is far from obvious that the strict concavity of $\tau^{*}(q)$ implies the multifractal scaling of the probability measure constructed from the critical wave function for a given realization of the disorder, i.e., the strict concavity of $\tau(q)$, as is implicitly assumed in Refs. [15, 14, 11, 20]. The important issue of how to calculate the universal scaling exponents $\{\tau(q) \mid q \in \mathbb{N}\}$ within our critical field theory remains open, since the scaling exponents $\left\{\Delta_{q} \mid q \in \mathbb{N}\right\}$ are not simply related to the $\tau(q)$ 's. An attempt to relate the $\Delta_{q}$ 's to the $\tau(q)$ 's will be published elsewhere 38 .

On the other hand, scaling with respect to spatial separation and effective system size of the averaged two-point correlation between the $q$-th generalized inverse participation ratio and the $r$-th generalized inverse participation ratio agrees in form with the one proposed for non-normalizable box-observables obeying multifractal scaling [39, 11, 10]. Since the existence of negative dimensional operators is crucial to the former scaling, it is tempting to conjecture that multifractality of the critical wave function might be related to the existence of negative dimensional operators in the effective critical field theory.

To uncover the nature of the critical field theories with $g_{A} \geq 0, g_{B}=0, \infty$, we study their current algebras. Without loss of generality, we restrict ourselves to Dirac fermions with Abelian vector gauge-like disorder. We show that any disordered critical point $g_{A}>0$ realizes a Virasoro algebra with vanishing central charge. Moreover, we show that this Virasoro algebra is obtained from currents obeying a $U(1 / 1) \times U(1 / 1)$ graded Kac-Moody algebra. The vanishing of the Virasoro central charge follows from using an effective partition function which is chosen to be unity in order to perform averages over the disorder. The Kac-Moody currents must obey a $U(1 / 1) \times U(1 / 1)$ graded algebra, since, on the one hand, the Hamiltonian for our random Dirac fermions can be interpreted as a Lagrangian in two-dimensional Euclidean space with $U(1) \times U(1)$ chiral symmetry, and, on the other hand, bosonic copies of the Dirac fermions have been introduced to perform averages over disorder. After having introduced bosonic copies of Dirac fermions, the effective field theory cannot be unitary anymore. All unusual properties of the disordered critical point $g_{A}>0$ can be traced to the bosonic sector of the effective field theory.

Having identified the underlying current algebras for the disordered critical points $g_{A}>0$, we find new critical points by considering more general current algebras. We simply require that the critical points be described by two-dimensional Conformal Field Theories (CFT's) [40] with vanishing central charges and obeying $U(1 / 1) \times U(1 / 1)$ Kac-Moody current algebras. All such critical points are classified in terms of three numbers $\left(l, m, k_{j}\right)$. For odd integer $l$, the spin of Dirac spinors, which is $\frac{1}{2}$ in the absence of disorder, remains half-integer as the $U(1 / 1) \times U(1 / 1)$ symmetric interaction is switched on. For any real $m$ and $k_{j}$, the Virasoro algebra constructed from the currents generating the $U(1 / 1) \times U(1 / 1)$ Kac-Moody algebra has vanishing central charge: a necessary condition to describe disordered criticality. Finally, the quantization of $m$ follows from the requirement that the CFT be local. The Dirac fermions with Abelian vector gauge-like disorder provide a Lagrangian realization of the CFT with

$$
\left(l, m, k_{j}\right)=\left(1,1, \frac{4 g_{A}}{\pi}\right) .
$$

We also construct Lagrangian realizations for all other CFT's. However, whether or not the remaining critical points describe criticality in systems of disordered non-interacting electrons is still an open question.

For each critical point $\left(l, m, k_{j}\right)$, we obtain the operator content from the current algebra. We show, for example, that there exists an infinite hierarchy of local operators transforming like $U(1 / 1) \times U(1 / 1)$ singlets with the spectrum of scaling exponents

$$
\Delta_{q}^{\left(l, m, k_{j}\right)}=m q+q^{2}(l-m)-q^{2}\left[l-m^{2}\left(1-\frac{k_{j}}{4}\right)\right], \quad q \in \mathbb{N} .
$$

Note that Eq. (1.1) is recovered for $\left(l, m, k_{j}\right)$ given by Eq. (1.4). Conditions on $l, m$, and $k_{j}$ to insure that no operator with negative scaling dimensions be present at criticality are easy to obtain. When operators with negative scaling dimensions are present in the operator content and are not forbidden by some additional symmetry requirement, the critical point is unstable in the special way described above. 


\section{B. Outline}

The paper is organized as follows. We briefly review the supersymmetric representation of single-particle Green functions in the theory of localization in section II. The operator content of Dirac fermions in the presence of Abelian and non-Abelian vector gauge-like randomness is derived in sections III and IV] respectively. The scaling with energy of the averaged local density of states, averaged generalized inverse participation ratios, and of the averaged spatial correlations of generalized inverse participation ratios is derived in section $\mathrm{V}$. In section VI, we study the algebraic structure of Conformal Field Theories related to Dirac fermions in the presence of vector gauge-like disorder. This section can be read independently from the previous ones. We conclude with a discussion of implications from our work for the plateau transition and the random $X Y$ model. In appendix A we give a detailed proof of the Sugawara construction of section VI. Our last appendix B is devoted to Lagrangian realizations of the CFT's constructed in section VI.

\section{AVERAGED PRODUCT OF SINGLE-PARTICLE GREEN FUNCTIONS}

We are concerned with calculating the disorder average over arbitrary products of matrix elements of the resolvent

$$
G(x, y, \omega \pm \mathrm{i} \eta ; V) \equiv\left\langle x\left|\frac{1}{\omega-H_{0}-V \pm \mathrm{i} \eta}\right| y\right\rangle, \quad \eta>0 .
$$

Here, $H_{0}$ is any single-particle Hamiltonian in the absence of disorder, whereas $V$ is any static single-particle potential representing disorder. Both are assumed to be local in space. The limit $\eta \rightarrow 0^{+}$gives the retarded $(+)$and advanced $(-)$ single-particle Green functions in the energy representation for a given realization $V$ of the disorder in a statistical ensemble with probability distribution $\mathcal{P}[V]$. The coordinates $x, y$ always denote spatial coordinates. They also implicitly denote degenerate internal degrees of freedom, unless explicitly stated.

Impurity average over products of single-particle Green functions is defined by

$$
\begin{aligned}
& \frac{\Gamma^{(m, n)}\left(\left\{x_{k}, y_{k}, \omega_{k} \pm \mathrm{i} \eta\right\}\right) \equiv}{\left(\prod_{i=1}^{m} G\left(x_{i}, y_{i}, \omega_{i}+\mathrm{i} \eta ; V\right)\right)\left(\prod_{j=1}^{n} G\left(x_{m+j}, y_{m+j}, \omega_{m+j}-\mathrm{i} \eta ; V\right)\right)} \equiv \\
& \int \mathcal{D}[V] \mathcal{P}[V]\left(\prod_{i=1}^{m} G\left(x_{i}, y_{i}, \omega_{i}+\mathrm{i} \eta ; V\right)\right)\left(\prod_{j=1}^{n} G\left(x_{m+j}, y_{m+j}, \omega_{m+j}-\mathrm{i} \eta ; V\right)\right)
\end{aligned}
$$

We will be mostly interested in the averaged density of states per volume and per energy

$$
\rho(\omega) \equiv \lim _{\eta \rightarrow 0^{+}} \frac{1}{2 \pi \mathrm{i}} \overline{\operatorname{tr}[G(x, x, \omega-\mathrm{i} \eta ; V)-G(x, x, \omega+\mathrm{i} \eta ; V)]},
$$

the impurity average over the $q$-th power of the smeared density of states per volume and per energy

$$
A^{(q)}(\omega, \eta) \equiv(2 \pi \mathrm{i})^{-q} \overline{\{\operatorname{tr}[G(x, x, \omega-\mathrm{i} \eta ; V)-G(x, x, \omega+\mathrm{i} \eta ; V)]\}^{q}},
$$

and the impurity average over the product

$$
\Gamma^{(1,1)}(x, y, \omega+\mathrm{i} \eta ; y, x, \omega-\mathrm{i} \eta) \equiv \overline{G(x, y, \omega+\mathrm{i} \eta ; V) G(y, x, \omega-\mathrm{i} \eta ; V)} .
$$

The trace is performed over any internal degrees of freedom such as spin, orbital degeneracy, etc. Averaging over randomness is assumed to restore any spatial homogeneity which can be broken by a particular member $V$ of the statistical ensemble. Hence, $\rho(\omega)$ and $A^{(q)}(\omega, \eta)$ are assumed not to depend on $x$. There exists an important Ward identity [4]

$$
\begin{aligned}
& \int d^{d} x \lim _{\eta \rightarrow 0^{+}} \eta \overline{|\operatorname{tr} G(x, y, \omega+\mathrm{i} \eta ; V)|^{2}}=\pi \rho_{\mathrm{loc}}(\omega), \\
& \lim _{\eta \rightarrow 0^{+}} \eta \int d^{d} x \overline{|\operatorname{tr} G(x, y, \omega+\mathrm{i} \eta ; V)|^{2}}=\pi \rho(\omega) .
\end{aligned}
$$


In Eq. (2.6a), the limit $\eta \rightarrow 0^{+}$is taken before integrating over space. States which diffuse at infinity do not contribute to the right-hand-side of Eq. (2.6a) for any finite separation $|x-y|$. These are the extended states. The only contributions in Eq. (2.6a) come from localized states. On the other hand, both localized and extended states contribute in Eq. (2.6b). We will always be working in the thermodynamic limit, i.e., we will be concerned with the order of limits of Eq. (2.6b).

\section{A. Graded supersymmetric treatment of the disorder}

In this subsection, we recall how the representation of the single-particle Green functions in terms of a path integral over anticommuting (Grassmann) and commuting variables allows to perform the impurity average over the resolvent before taking its matrix elements [28]. To this end, we simply use the Gaussian integrals

$$
\begin{aligned}
& \int d \psi^{*} d \psi e^{-\psi^{*} a \psi}=a, \quad \forall a \text { complex, } \\
& \int d \psi^{*} d \psi \psi \psi^{*} e^{-\psi^{*} b \psi}=1, \quad \forall b \text { complex, } \\
& \frac{\int d \psi^{*} d \psi \psi \psi^{*} e^{-\psi^{*} b \psi}}{\int d \psi^{*} d \psi e^{-\psi^{*} a \psi}}=\frac{1}{a}, \quad \forall a, b \text { complex },
\end{aligned}
$$

for the pair $\psi^{*}, \psi$ of Grassmann variables, and

$$
\begin{aligned}
& \int d \varphi^{*} d \varphi e^{-\varphi^{*} a \varphi}=\frac{1}{a}, \quad \operatorname{Re} a>0, \\
& \int d \varphi^{*} d \varphi \varphi^{*} \varphi e^{-\varphi^{*} a \varphi}=\frac{1}{a} \int d \varphi^{*} d \varphi e^{-\varphi^{*} a \varphi}, \quad \operatorname{Re} a>0, \\
& \frac{\int d \varphi^{*} d \varphi \varphi^{*} \varphi e^{-\varphi^{*} a \varphi}}{\int d \varphi^{*} d \varphi e^{-\varphi^{*} a \varphi}}=\frac{1}{a}, \quad \operatorname{Re} a>0,
\end{aligned}
$$

for the pair $\varphi^{*}, \varphi$ of complex variables and generalize them to Gaussian integrals for fermionic coherent states and bosonic coherent states, respectively.

In this way, the denominator in the fermionic path integral representation

$$
G(x, y, \omega \pm \mathrm{i} \eta ; V)=\mp \mathrm{i} \frac{\int \mathcal{D} \psi^{*} \mathcal{D} \psi \psi(x) \psi^{*}(y) e^{+\mathrm{i} \int \psi^{*}\left[( \pm)\left(\omega-H_{0}-V\right)+\mathrm{i} \eta\right] \psi}}{\int \mathcal{D} \psi^{*} \mathcal{D} \psi e^{+\mathrm{i} \int \psi^{*}\left[( \pm)\left(\omega-H_{0}-V\right)+\mathrm{i} \eta\right] \psi}}
$$

can be rewritten as a Gaussian integrals over bosonic coherent states:

$$
\begin{aligned}
& G(x, y, \omega \pm \mathrm{i} \eta ; V)= \\
& \mp \mathrm{i} \int \mathcal{D} \psi^{*} \mathcal{D} \psi \int \mathcal{D} \varphi^{*} \mathcal{D} \varphi \psi(x) \psi^{*}(y) e^{+\mathrm{i} \int \psi^{*}\left[( \pm)\left(\omega-H_{0}-V\right)+\mathrm{i} \eta\right] \psi+\mathrm{i} \int \varphi^{*}\left[( \pm)\left(\omega-H_{0}-V\right)+\mathrm{i} \eta\right] \varphi} .
\end{aligned}
$$

We could have equally well chosen the bosonic path integral representation

$$
G(x, y, \omega \pm \mathrm{i} \eta ; V)=\mp \mathrm{i} \frac{\int \mathcal{D} \varphi^{*} \mathcal{D} \varphi \varphi(x) \varphi^{*}(y) e^{+\mathrm{i} \int \varphi^{*}\left[( \pm)\left(\omega-H_{0}-V\right)+\mathrm{i} \eta\right] \varphi}}{\int \mathcal{D} \varphi^{*} \mathcal{D} \varphi e^{+\mathrm{i} \int \varphi^{*}\left[( \pm)\left(\omega-H_{0}-V\right)+\mathrm{i} \eta\right] \varphi}},
$$

which can be rewritten as

$$
\begin{aligned}
& G(x, y, \omega \pm \mathrm{i} \eta ; V)= \\
& \mp \mathrm{i} \int \mathcal{D} \psi^{*} \mathcal{D} \psi \int \mathcal{D} \varphi^{*} \mathcal{D} \varphi \varphi(x) \varphi^{*}(y) e^{+\mathrm{i} \int \psi^{*}\left[( \pm)\left(\omega-H_{0}-V\right)+\mathrm{i} \eta\right] \psi+\mathrm{i} \int \varphi^{*}\left[( \pm)\left(\omega-H_{0}-V\right)+\mathrm{i} \eta\right] \varphi} .
\end{aligned}
$$

Eqs. (2.9b) and $(2.10 \mathrm{~b})$ can be combined into 


$$
\begin{aligned}
G(x, y, \omega \pm \mathrm{i} \eta ; V) & =\mp \mathrm{i} \int \mathcal{D} \psi^{*} \mathcal{D} \psi \int \mathcal{D} \varphi^{*} \mathcal{D} \varphi \frac{\left[\psi(x) \psi^{*}(y)+\varphi(x) \varphi^{*}(y)\right]}{2} \\
& \times e^{+\mathrm{i} \int \psi^{*}\left[( \pm)\left(\omega-H_{0}-V\right)+\mathrm{i} \eta\right] \psi+\mathrm{i} \int \varphi^{*}\left[( \pm)\left(\omega-H_{0}-V\right)+\mathrm{i} \eta\right] \varphi} .
\end{aligned}
$$

Eqs. (2.9b, 2.10b, 2.11) are graded supersymmetric representations of the matrix elements of the resolvent. They require $H_{0}+V$ to be quadratic in the fermionic $(\psi)$ or bosonic $(\varphi)$ coherent states and thus can only be used when the pure system is non-interacting.

In the graded supersymmetric representation, average over the statistical ensemble of impurity potential can be performed before calculating the matrix elements of the resolvent, and by so doing, averaged matrix elements of the resolvent are obtained from Green functions of an effective interacting theory for fermionic and bosonic coherent states. The effective interaction resulting from performing first the impurity average depends on two distinct properties of the impurities. One property is the nature of the coupling between the impurities and the single-particle eigenstates of $H_{0}$. It is the same for all impurity potentials in the statistical ensemble. The second property is the probability distribution $\mathcal{P}[V]$ for the impurity potential. The scaling hypothesis in the theory of localization assumes that given one type of impurity potential, there can exist a critical energy $\omega_{c}$, the so-called mobility edge. Upon approaching this critical energy, the scaling hypothesis assumes the existence of one relevant diverging length scale. Whereas the existence of the mobility edge is determined by the nature of the coupling between the impurities and the singleparticle eigenstates of $H_{0}$, the microscopic properties of the probability distribution $\mathcal{P}[V]$ should be unobservable close to the mobility edge. In other words, properties of $\mathcal{P}[V]$ on length scales much smaller than the diverging relevant length scale should not be observable. It is then natural to consider white noise probability distributions, i.e., statistical ensembles such that

$$
\overline{V_{a}(x) V_{b}(y)}=v^{2} \delta_{a b} \delta(x-y),
$$

where $a, b$ collectively denote degrees of freedom such as spin, orbital degeneracy, and so on, to describe a statistical ensemble with short-range spatial correlations. The energy scale $v$ then alone characterizes the impurity strength. In the spirit of the scaling hypothesis, it is usually assumed that close to the mobility edge the choice of a Gaussian probability distribution,

$$
\mathcal{P}[V] \propto \prod_{x} e^{-\frac{1}{2 v^{2}} \operatorname{tr}\left[V^{2}(x)\right]},
$$

can be made without loss of generality. Averaging over the impurity potentials then amounts to simple Gaussian integrals inducing an effective interaction which is quartic in the coherent fermionic and bosonic fields. In the graded supersymmetric representation, the average defined in Eq. (2.2) becomes the $2(m+n)$-point Green function

$$
\Gamma^{(m, n)}\left(\left\{x_{k}, y_{k}, \omega_{k} \pm \mathrm{i} \eta\right\}\right)=\mathrm{i}^{n-m}\left\langle\left(\prod_{i=1}^{m} \psi_{i}\left(x_{i}\right) \psi_{i}^{*}\left(y_{i}\right)\right)\left(\prod_{j=1}^{n} \psi_{m+j}\left(x_{m+j}\right) \psi_{m+j}^{*}\left(y_{m+j}\right)\right)\right\rangle .
$$

Here, the expectation value is taken with respect to the partition function $\mathcal{Z}_{\text {eff }}^{(m, n)}$ defined by the weight

$$
\begin{aligned}
& e^{\mathrm{i} S_{\mathrm{eff}}^{(m, n)}\left(\left\{\omega_{k} \pm \mathrm{i} \eta\right\}\right)} \equiv \int \mathcal{D}[V] \mathcal{P}[V] e^{\mathrm{i} S_{\mathrm{r}}^{(m)}+\mathrm{i} S_{\mathrm{a}}^{(n)}-S_{\eta}^{(m+n)}}, \\
& S_{\mathrm{r}}^{(m)}=+\sum_{i=1}^{m} \int d^{d} x\left[\psi_{i}^{*}\left(\omega_{i}-H_{0}-V\right) \psi_{i}+\varphi_{i}^{*}\left(\omega_{i}-H_{0}-V\right) \varphi_{i}\right], \\
& S_{\mathrm{a}}^{(n)}=-\sum_{j=1}^{n} \int d^{d} x\left[\psi_{m+j}^{*}\left(\omega_{m+j}-H_{0}-V\right) \psi_{m+j}+\left(\psi^{*}, \psi\right) \rightarrow\left(\varphi^{*}, \varphi\right)\right], \\
& S_{\eta}^{(m+n)}=\eta \sum_{k=1}^{m+n} \int d^{d} x\left[\psi_{k}^{*} \psi_{k}+\varphi_{k}^{*} \varphi_{k}\right],
\end{aligned}
$$

and the measure

$$
\mathcal{D}\left[\psi^{*}, \psi, \varphi^{*}, \varphi\right]=\prod_{x} \prod_{k=1}^{m+n} d \psi_{k}^{*}(x) d \psi_{k}(x) d \varphi_{k}^{*}(x) d \varphi_{k}(x)
$$


Notice that by construction $\mathcal{Z}_{\mathrm{eff}}^{(m, n)}=1$.

The action $S_{\eta}^{(m+n)}$ insures the convergence of the path integral. It also has the largest symmetry, being diagonal in all degrees of freedom. In particular, it possesses a local $U(m+n)$ symmetry where $m$ and $n$ represent the number of retarded and advanced single-particle Green functions over which the impurity average is performed. The action for the retarded sector $S_{\mathrm{r}}^{(m)}$ has a smaller global $U(m)$ symmetry when all energies $\omega_{i}, i=1, \cdots, m$ are equal. The same holds in the advanced sector $S_{\mathrm{a}}^{(n)}$ with $n$ replacing $m$. Hence, in the special case

$$
\omega_{1}=\cdots=\omega_{m}=\omega_{\mathrm{r}}, \quad \omega_{m+1}=\cdots=\omega_{m+n}=\omega_{\mathrm{a}}
$$

the effective action $S_{\mathrm{eff}}^{(m, n)}$ is symmetric under global $U(m) \times U(n)$ transformations acting on the index $k=1, \cdots, m+n$ of the coherent states. Another important property of the effective action $S_{\mathrm{eff}}^{(m, n)}$ is that when all energies are set to zero in the single-particle Green functions:

$$
0=\omega_{1}=\cdots=\omega_{m+n}
$$

then $S_{\mathrm{r}}^{(m)}+S_{\mathrm{a}}^{(n)}$ is invariant under the group $U(m, n)$ which leaves

$$
\operatorname{diag}(\underbrace{+1, \cdots,+1}_{m}, \underbrace{-1, \cdots,-1}_{n})
$$

unchanged.

\section{ABELIAN VECTOR GAUGE RANDOMNESS}

From now on, we restrict the generality of our discussion to two spatial dimensions and to the single-particle Hamiltonian describing one species of massless Dirac fermions in the presence of static Abelian vector gauge-like randomness 20]. In other words, the Hamiltonian of the pure system is the two by two matrix

$$
H_{0}=-\mathrm{i} \gamma_{\mu} \partial_{\mu} .
$$

Here, $\mu=1,2$ denote the two spatial directions and $\gamma_{1}, \gamma_{2}$ are any two of the three Pauli matrices, $\gamma_{5}=-\mathrm{i} \gamma_{1} \gamma_{2}$ being the third. The coupling of the impurities to the single-particle states is through the dimensionfull vector gauge-like potential $A_{\mu}(x)$ :

$$
V(x)=-\sqrt{g_{A}} A_{\mu}(x) \gamma_{\mu} .
$$

The probability distribution for the random vector gauge-like disorder is Gaussian:

$$
\mathcal{P}\left[A_{\mu}\right] \propto e^{-\frac{1}{2} \int d^{2} x A_{\mu}^{2}(x)} .
$$

In the graded supersymmetric path integral representation of the Green functions, the fermionic coherent states are denoted $(\bar{\psi}, \psi)$, while the bosonic coherent states are denoted $(\bar{\varphi}, \varphi)$. Both fermionic and bosonic coherent states transform like Dirac spinors in two-dimensional Euclidean space, and thus implicitly carry spinor indices. Eq. (2.14) becomes

$$
\begin{aligned}
& \Gamma^{(m, n)}\left(\left\{x_{k}, y_{k}, \omega_{k} \pm \mathrm{i} \eta\right\}\right)=\mathrm{i}^{n-m}\left\langle\left(\prod_{i=1}^{m} \psi_{i}\left(x_{i}\right) \bar{\psi}_{i}\left(y_{i}\right)\right)\left(\prod_{j=1}^{n} \psi_{m+j}\left(x_{m+j}\right) \bar{\psi}_{m+j}\left(y_{m+j}\right)\right)\right\rangle \\
& e^{\mathrm{i} S_{\mathrm{eff}}^{(m, n)}\left(\left\{\omega_{k} \pm \mathrm{i} \eta\right\}\right)} \equiv \int \mathcal{D}\left[A_{\mu}\right] e^{-\frac{1}{2} \int d^{2} x A_{\mu}^{2}(x)+\mathrm{i} S_{\mathrm{r}}^{(m)}+\mathrm{i} S_{\mathrm{a}}^{(n)}-S_{\eta}^{(m+n)}}, \\
& S_{\mathrm{r}}^{(m)}=+\sum_{i=1}^{m} \int d^{2} x\left\{\bar{\psi}_{i}\left[\mathrm{i} \gamma_{\mu}\left(\partial_{\mu}-\mathrm{i} \sqrt{g}_{A} A_{\mu}\right)+\omega_{i}\right] \psi_{i}+(\bar{\psi}, \psi) \rightarrow(\bar{\varphi}, \varphi)\right\} \\
& S_{\mathrm{a}}^{(n)}=-\sum_{j=1}^{n} \int d^{2} x\left\{\bar{\psi}_{m+j}\left[\mathrm{i} \gamma_{\mu}\left(\partial_{\mu}-\mathrm{i} \sqrt{g_{A}} A_{\mu}\right)+\omega_{m+j}\right] \psi_{m+j}+(\bar{\psi}, \psi) \rightarrow(\bar{\varphi}, \varphi)\right\} \\
& S_{\eta}^{(m+n)}=\eta \sum_{k=1}^{m+n} \int d^{2} x\left(\bar{\psi}_{k} \psi_{k}+\bar{\varphi}_{k} \varphi_{k}\right) \\
& \mathcal{D}[\bar{\psi}, \psi, \bar{\varphi}, \varphi]=\prod_{k=1}^{m+n} \mathcal{D} \bar{\psi}_{k} \mathcal{D} \psi_{k} \mathcal{D} \bar{\varphi}_{k} \mathcal{D} \varphi_{k} .
\end{aligned}
$$


Integrating over the random vector gauge-like potential induces a quartic coupling mixing all the indices labelling the $m+n$ single-particle Green functions. It would therefore appear that the effective action $S_{\text {eff }}^{(m+n)}$ is hopelessly complicated. This is not so however in a very special case, namely when all energy scales are set to zero:

$$
\omega_{1}=\cdots=\omega_{m+n}=0, \quad \eta=0 .
$$

Indeed it is then possible to perform a very simple trick to decouple entirely the Dirac spinors from the random vector gauge-like potential. To see this, we first make use of the fact that, since $x$ belongs to a two-dimensional manifold taken to be the Euclidean plane and since the vector potential $\left(A_{1}(x), A_{2}(x)\right)$ has only two components, $A_{\mu}$ can always be decomposed into a transversal $\Phi_{1}(x)$ and a longitudinal $\Phi_{2}(x)$ component, provided $A_{\mu}$ is free of singularities associated with configurations of magnetic monopoles. Accordingly, we can use the Hodge decomposition

$$
A_{\mu}(x)=\tilde{\partial}_{\mu} \Phi_{1}(x)+\partial_{\mu} \Phi_{2}(x), \quad \tilde{\partial}_{\mu} \equiv \epsilon_{\mu \nu} \partial_{\nu} .
$$

We have used the completely antisymmetric second rank (Levi-Cevita) tensor $\epsilon_{\mu \nu}$ with $\epsilon_{12}=1$. Parametrizing the random vector gauge-like fields in terms of their transversal and longitudinal components induces two changes in their probability distribution. The first one comes from the Jacobian induced by the change of variables. The second one comes from the change in their probability distribution. We thus have 42

$$
\begin{aligned}
\mathcal{D}\left[A_{\mu}\right] \mathcal{P}\left[A_{\mu}\right] & =\mathcal{D}\left[\Phi_{\mu}\right] \mathcal{P}\left[\Phi_{\mu}\right] \operatorname{Det}\left[\partial^{2}\right] \\
& =\mathcal{D}\left[\Phi_{\mu}\right] e^{-\frac{1}{2} \int d^{2} x\left[\left(\partial_{\mu} \Phi_{1}\right)^{2}+\left(\partial_{\mu} \Phi_{2}\right)^{2}\right]} \int \mathcal{D}[\bar{\alpha}, \alpha] e^{-\int d^{2} x \bar{\alpha} \partial^{2} \alpha} .
\end{aligned}
$$

We are implicitly assuming that the vector gauge fields vanish at infinity. On the last line, the Jacobian of the transformation is rewritten in terms of a path integral over fermionic coherent states $(\bar{\alpha}, \alpha)$. We will refer to these degrees of freedom as ghost fields. They do not play an important role for an Abelian vector gauge impurity potential, but they must be accounted for in the case of non-Abelian vector gauge impurity potentials as we will see later on. The reason for which Abelian ghosts are not important is that the Jacobian in Eq. (3.7) is independent of the Abelian vector gauge-like configuration $A_{\mu}$.

After the change of variables Eq. (3.6), the minimal coupling between the Dirac spinors and the impurity potential takes the form

$$
\bar{\psi}_{k} \gamma_{\mu}\left[\partial_{\mu}-\mathrm{i} \sqrt{g_{A}}\left(\left(\tilde{\partial}_{\mu} \Phi_{1}\right)+\left(\partial_{\mu} \Phi_{2}\right)\right)\right] \psi_{k}, \quad k=1, \cdots, m+n,
$$

in the fermionic coherent state sector, and

$$
\bar{\varphi}_{k} \gamma_{\mu}\left[\partial_{\mu}-\mathrm{i} \sqrt{g_{A}}\left(\left(\tilde{\partial}_{\mu} \Phi_{1}\right)+\left(\partial_{\mu} \Phi_{2}\right)\right)\right] \varphi_{k}, \quad k=1, \cdots, m+n,
$$

in the bosonic coherent state sector. Again we make use of the fact that we are effectively working with covariant gauge fields defined on a two-dimensional manifold so that the important identity

$$
\gamma_{\mu} \gamma_{5}=\mathrm{i} \epsilon_{\mu \nu} \gamma_{\nu}, \quad \mu=1,2
$$

holds. It is then easy to verify that for the $k$-th Green function in the product $\Gamma^{(m, n)}$, the change of variables

$$
\begin{aligned}
& \bar{\psi}_{k}=\bar{\psi}_{k}^{\prime} e^{\gamma_{5} \sqrt{g_{A}} \Phi_{1}-\mathrm{i} \sqrt{g_{A}} \Phi_{2}}, \quad \psi_{k}=e^{\gamma_{5} \sqrt{g_{A}} \Phi_{1}+\mathrm{i} \sqrt{g_{A}} \Phi_{2}} \psi_{k}^{\prime}, \\
& \bar{\varphi}_{k}=\bar{\varphi}_{k}^{\prime} e^{\gamma_{5} \sqrt{g_{A}} \Phi_{1}-\mathrm{i} \sqrt{g_{A}} \Phi_{2}}, \quad \varphi_{k}=e^{\gamma_{5} \sqrt{g_{A}} \Phi_{1}+\mathrm{i} \sqrt{g_{A}} \Phi_{2}} \varphi_{k}^{\prime},
\end{aligned}
$$

decouples the Abelian vector gauge-like fields from the Dirac spinors 443. This local transformation can be repeated for all $m+n$ single-particle Green functions. It is not a pure gauge transformation since it involves the longitudinal component $\Phi_{1}$. It is the product of a pure gauge transformation (the exponent $\Phi_{2}$ ) with an axial transformation (the exponent $\left.\Phi_{1}\right)$.

The axial factor of the decoupling transformation Eq. (3.10) is non-trivial in many respects. First, in contrast to the pure gauge factor, it does not leave the terms proportional to the energy scales $\omega_{k}, \eta$ unchanged:

$$
\bar{\psi}_{k} \psi_{k}=\bar{\psi}_{k}^{\prime} e^{2 \gamma_{5} \sqrt{g_{A}} \Phi_{1}} \psi_{k}^{\prime}, \quad \bar{\varphi}_{k} \varphi_{k}=\bar{\varphi}_{k}^{\prime} e^{2 \gamma_{5} \sqrt{g_{A}} \Phi_{1}} \varphi_{k}^{\prime}, \quad k=1, \cdots, m+n .
$$

Second, it does induce a non-trivial Jacobian for both the fermionic and bosonic coherent state measures, as long as a fully gauge invariant regularization of these measures is used [14]. However, the Jacobian induced from the fermionic measure $[45]$ 


$$
\mathcal{D}[\bar{\psi}, \psi]=\mathcal{D}\left[\bar{\psi}^{\prime}, \psi^{\prime}\right] e^{-\frac{1}{2 \pi} \int d^{2} x\left(\partial_{\mu} \Phi_{1}\right)^{2}}
$$

cancels that from the bosonic measure (this cancellation does not hold for non-Abelian gauge fields due to the ghost sector). Finally, the manifold defined by all axial transformations is not compact.

The impurity average of any product of single-particle Green functions factorizes when all their energies are set to zero:

$$
\begin{aligned}
& \lim _{\eta \rightarrow 0^{+}} \Gamma^{(m, n)}\left(\left\{x_{k}, y_{k}, \pm \mathrm{i} \eta\right\}\right)= \\
& \mathrm{i}^{n-m}\left\langle\left(\prod_{i=1}^{m} \psi_{i}^{\prime}\left(x_{i}\right) \bar{\psi}_{i}^{\prime}\left(y_{i}\right)\right)\left(\prod_{j=1}^{n} \psi_{m+j}^{\prime}\left(x_{m+j}\right) \bar{\psi}_{m+j}^{\prime}\left(y_{m+j}\right)\right)\right\rangle_{\bar{\psi}^{\prime}, \psi^{\prime}, \bar{\varphi}^{\prime}, \varphi^{\prime}} \\
& \times\left\langle\left(\prod_{i=1}^{m} e^{\gamma_{5} \Phi_{1}\left(x_{i}\right)} e^{\gamma_{5} \Phi_{1}\left(y_{i}\right)}\right)\left(\prod_{j=1}^{n} e^{\gamma_{5} \Phi_{1}\left(x_{m+j}\right)} e^{\gamma_{5} \Phi_{1}\left(y_{m+j}\right)}\right)\right\rangle_{\Phi_{1}, \bar{\alpha}, \alpha} \\
& \times\left\langle\left(\prod_{i=1}^{m} e^{+\mathrm{i} \Phi_{2}\left(x_{i}\right)} e^{-\mathrm{i} \Phi_{2}\left(y_{i}\right)}\right)\left(\prod_{j=1}^{n} e^{+\mathrm{i} \Phi_{2}\left(x_{m+j}\right)} e^{-\mathrm{i} \Phi_{2}\left(y_{m+j}\right)}\right)\right\rangle_{\Phi_{2}} .
\end{aligned}
$$

The expectation value with respect to the spinor coherent states is 2

$$
\begin{aligned}
& \langle f\rangle_{\bar{\psi}^{\prime}, \psi^{\prime}, \bar{\varphi}^{\prime}, \varphi^{\prime}}=\int \mathcal{D}\left[\bar{\psi}^{\prime}, \psi^{\prime}, \bar{\varphi}^{\prime}, \varphi^{\prime}\right] e^{\mathrm{i} S_{\mathrm{r}}^{\prime(m)}+\mathrm{i} S_{\mathrm{a}}^{\prime(n)}} f, \\
& S_{\mathrm{r}}^{\prime(m)}=+\sum_{i=1}^{m} \int d^{2} x\left(\bar{\psi}_{i}^{\prime} \mathrm{i} \gamma_{\mu} \partial_{\mu} \psi_{i}^{\prime}+\bar{\varphi}_{i}^{\prime} \mathrm{i} \gamma_{\mu} \partial_{\mu} \varphi_{i}^{\prime}\right), \\
& S_{\mathrm{a}}^{\prime(n)}=-\sum_{j=1}^{n} \int d^{2} x\left(\bar{\psi}_{m+j}^{\prime} \mathrm{i} \gamma_{\mu} \partial_{\mu} \psi_{m+j}^{\prime}+\bar{\varphi}_{m+j}^{\prime} \mathrm{i} \gamma_{\mu} \partial_{\mu} \varphi_{m+j}^{\prime}\right) .
\end{aligned}
$$

The expectation value with respect to the longitudinal component of the Abelian gauge potential is

$$
\langle f\rangle_{\Phi_{1}}=\int \mathcal{D}\left[\Phi_{1}\right] e^{-S_{\mathrm{imp}}\left[\Phi_{1}\right]} f, \quad S_{\mathrm{imp}}\left[\Phi_{1}\right]=\frac{1}{2} \int d^{2} x\left(\partial_{\mu} \Phi_{1}\right)^{2} .
$$

The expectation value with respect to the the transversal component of the Abelian gauge potential is

$$
\langle f\rangle_{\Phi_{2}}=\int \mathcal{D}\left[\Phi_{2}\right] e^{-S_{\mathrm{imp}}\left[\Phi_{2}\right]} f, \quad S_{\mathrm{imp}}\left[\Phi_{2}\right]=\frac{1}{2} \int d^{2} x\left(\partial_{\mu} \Phi_{2}\right)^{2} .
$$

Finally, there is an innocuous average over the ghost coherent states

$$
\langle f\rangle_{\bar{\alpha}, \alpha}=\int \mathcal{D}[\bar{\alpha}, \alpha] e^{\mathrm{i} S_{\mathrm{gh}}} f, \quad S_{\mathrm{gh}}=\int d^{2} x \bar{\alpha} \mathrm{i} \partial^{2} \alpha .
$$

Thus, the impurity average of any product of single-particle Green functions can be obtained from the correlation functions generated by the corresponding critical Euclidean effective action

$$
S_{\mathrm{cr}}^{(m, n)} \equiv S_{\mathrm{imp}}\left[\Phi_{1}\right]+S_{\mathrm{imp}}\left[\Phi_{2}\right]-\mathrm{i} S_{\mathrm{gh}}-\mathrm{i} S_{\mathrm{r}}^{\prime(m)}-\mathrm{i} S_{\mathrm{a}}^{\prime(n)} .
$$

The impurity strength does not appear explicitly, being hidden in the rotation from the original fields to the new ones. The effective action is critical in the sense that all correlation functions are algebraic as we will see below.

From the factorization in Eq. (3.13) follows immediately that given $m+n$ pairs of points $x_{k}, y_{k}$ on the Euclidean plane, the $2(m+n)$-point correlation function $\Gamma^{(m, n)}\left(\left\{x_{k}, y_{k}, 0\right\}\right)$ can be calculated by using any effective action $S_{\mathrm{cr}}^{(p, q)}$ as long as $p \geq m$ and $q \geq n$. For example, the average local density of states $\lim _{\omega \rightarrow 0} \rho(\omega)$ can be calculated from all effective actions $S_{\mathrm{cr}}^{(p, q)}$ with either $p \geq 1$ or $q \geq 1$. We now turn to the operator content of the effective actions $S_{\mathrm{cr}}^{(m, n)}$.

\footnotetext{
${ }^{2}$ Of course, integration over the bosonic coherent states is not guaranteed to converge anymore having set $\eta$ to zero. Hence, Eq. (3.13b is only a formal expression unless it is itself regularized.
} 


\section{A. Operator content at criticality for Abelian vector gauge randomness}

To study the operator content of the critical action in Eq. (3.14), it is useful to introduce the so-called chiral basis for Dirac spinors $\bar{\psi}, \psi, \bar{\varphi}, \varphi$. The chiral basis is defined by

$$
\begin{aligned}
\psi^{\dagger} \equiv \bar{\psi} \gamma_{1}, \quad \psi_{ \pm}^{\dagger} \equiv \psi^{\dagger} \frac{1}{2}\left(1 \pm \gamma_{5}\right), \quad \psi_{ \pm} \equiv \frac{1}{2}\left(1 \pm \gamma_{5}\right) \psi \\
\varphi^{\dagger} \equiv \bar{\varphi} \gamma_{1}, \quad \varphi_{ \pm}^{\dagger} \equiv \varphi^{\dagger} \frac{1}{2}\left(1 \pm \gamma_{5}\right), \quad \varphi_{ \pm} \equiv \frac{1}{2}\left(1 \pm \gamma_{5}\right) \varphi
\end{aligned}
$$

In the chiral basis, the decoupling transformation, Eq. (3.10), becomes

$$
\begin{aligned}
& \psi_{k \pm}^{\dagger}=\psi_{k \pm}^{\prime \dagger} e^{\mp \sqrt{g_{A}} \Phi_{1}-\mathrm{i} \sqrt{g_{A}} \Phi_{2}}, \quad \psi_{k \pm}=e^{ \pm \sqrt{g_{A}} \Phi_{1}+\mathrm{i} \sqrt{g_{A}} \Phi_{2}} \psi_{k \pm}^{\prime}, \quad k=1, \cdots, m+n, \\
& \varphi_{k \pm}^{\dagger}=\varphi_{k \pm}^{\prime \dagger} e^{\mp \sqrt{g_{A}} \Phi_{1}-\mathrm{i} \sqrt{g_{A}} \Phi_{2}}, \quad \varphi_{k \pm}=e^{ \pm \sqrt{g_{A}} \Phi_{1}+\mathrm{i} \sqrt{g_{A}} \Phi_{2}} \varphi_{k \pm}^{\prime}, \quad k=1, \cdots, m+n .
\end{aligned}
$$

The terms in Eq. (3.11) which couple to the energy in denominators of single-particle Green functions are represented by

$$
\begin{aligned}
& \bar{\psi}_{k} \psi_{k}=\psi_{k+}^{\prime \dagger} e^{-2 \sqrt{g_{A}} \Phi_{1}} \psi_{k-}^{\prime}+\psi_{k-}^{\prime \dagger} e^{+2 \sqrt{g_{A}} \Phi_{1}} \psi_{k+}^{\prime}, \quad k=1, \cdots, m+n, \\
& \bar{\varphi}_{k} \varphi_{k}=\varphi_{k+}^{\prime \dagger} e^{-2 \sqrt{g_{A}} \Phi_{1}} \varphi_{k-}^{\prime}+\varphi_{k-}^{\prime \dagger} e^{+2 \sqrt{g_{A}} \Phi_{1}} \varphi_{k+}^{\prime}, \quad k=1, \cdots, m+n,
\end{aligned}
$$

provided $\left(\gamma_{1}, \gamma_{2}, \gamma_{5}\right)$ are chosen to be the usual three Pauli matrices. The effective actions in the retarded and advanced Dirac spinor sectors take a simple form in the chiral basis. To see this, we first think of the Euclidean plane $\left\{x=\left(x_{1}, x_{2}\right) \mid x_{1}, x_{2} \in \mathbb{R}\right\}$ as being a real section of the two-dimensional complex manifold with local coordinates

$$
z=x_{1}+\mathrm{i} x_{2}, \quad \bar{z}=x_{1}-\mathrm{i} x_{2},
$$

where $x_{1}$ and $x_{2}$ are now arbitrary complex numbers. The effective actions in Eqs. (3.13c, 3.13d) now become

$$
\begin{aligned}
S_{\mathrm{r}}^{\prime(m)} & =+2 \mathrm{i} \sum_{i=1}^{m}\left(\psi_{i+}^{\prime \dagger} \partial_{\bar{z}} \psi_{i+}^{\prime}+\psi_{i-}^{\prime \dagger} \partial_{z} \psi_{i-}^{\prime}+\varphi_{i+}^{\prime \dagger} \partial_{\bar{z}} \varphi_{i+}^{\prime}+\varphi_{i-}^{\prime \dagger} \partial_{z} \varphi_{i-}^{\prime}\right), \\
S_{\mathrm{a}}^{\prime(n)} & =-2 \mathrm{i} \sum_{j=1}^{n}\left(\psi_{(m+j)+}^{\prime \dagger} \partial_{\bar{z}} \psi_{(m+j)+}^{\prime}+\psi_{(m+j)-}^{\prime \dagger} \partial_{z} \psi_{(m+j)-}^{\prime}+\left(\psi_{ \pm}^{\prime \dagger}, \psi_{ \pm}^{\prime}\right) \rightarrow\left(\varphi_{ \pm}^{\prime \dagger}, \varphi_{ \pm}^{\prime}\right)\right),
\end{aligned}
$$

respectively. The advantage of the chiral basis is that the two-point correlation functions in the decoupled spinor sectors are very simple. The only non-vanishing ones in the retarded sector are $(k, l=1, \cdots, m)$ :

$$
\begin{aligned}
\left\langle\psi_{k+}^{\prime}(z, \bar{z}) \psi_{l_{+}}^{\prime \dagger}(0,0)\right\rangle_{\psi_{ \pm}^{\prime \dagger}, \psi_{ \pm}^{\prime}, \varphi_{ \pm}^{\prime \dagger}, \varphi_{ \pm}^{\prime}} & =\left\langle\varphi_{k+}^{\prime}(z, \bar{z}) \varphi_{l+}^{\prime \dagger}(0,0)\right\rangle_{\psi_{ \pm}^{\prime \dagger}, \psi_{ \pm}^{\prime}, \varphi_{ \pm}^{\prime \dagger}, \varphi_{ \pm}^{\prime}}=\frac{\delta_{k l}}{2 \pi z}, \\
\left\langle\psi_{k-}^{\prime}(z, \bar{z}) \psi_{l_{-}}^{\prime \dagger}(0,0)\right\rangle_{\psi_{ \pm}^{\prime \dagger}, \psi_{ \pm}^{\prime}, \varphi_{ \pm}^{\prime \dagger}, \varphi_{ \pm}^{\prime}} & =\left\langle\varphi_{k-}^{\prime}(z, \bar{z}) \varphi_{l-}^{\prime \dagger}(0,0)\right\rangle_{\psi_{ \pm}^{\prime \dagger}, \psi_{ \pm}^{\prime}, \varphi_{ \pm}^{\prime \dagger}, \varphi_{ \pm}^{\prime}}=\frac{\delta_{k l}}{2 \pi \bar{z}},
\end{aligned}
$$

In the advanced sector, they are the same except for a relative $\operatorname{sign}(k, l=m+1, \cdots, m+n)$ :

$$
\begin{aligned}
\left\langle\psi_{k+}^{\prime}(z, \bar{z}) \psi_{l+}^{\prime \dagger}(0,0)\right\rangle_{\psi_{ \pm}^{\prime \dagger}, \psi_{ \pm}^{\prime}, \varphi_{ \pm}^{\prime \dagger}, \varphi_{ \pm}^{\prime}} & =\left\langle\varphi_{k+}^{\prime}(z, \bar{z}) \varphi_{l+}^{\prime \dagger}(0,0)\right\rangle_{\psi_{ \pm}^{\prime \dagger}, \psi_{ \pm}^{\prime}, \varphi_{ \pm}^{\prime \dagger}, \varphi_{ \pm}^{\prime}}=-\frac{\delta_{k l}}{2 \pi z} \\
\left\langle\psi_{k-}^{\prime}(z, \bar{z}) \psi_{l-}^{\prime \dagger}(0,0)\right\rangle_{\psi_{ \pm}^{\prime \dagger}, \psi_{ \pm}^{\prime}, \varphi_{ \pm}^{\prime \dagger}, \varphi_{ \pm}^{\prime}} & =\left\langle\varphi_{k-}^{\prime}(z, \bar{z}) \varphi_{l-}^{\prime \dagger}(0,0)\right\rangle_{\psi_{ \pm}^{\prime \dagger}, \psi_{ \pm}^{\prime}, \varphi_{ \pm}^{\prime \dagger}, \varphi_{ \pm}^{\prime}}=-\frac{\delta_{k l}}{2 \pi \bar{z}}
\end{aligned}
$$

Two-point functions between retarded and advanced spinor fields vanish. In the chiral basis, the two-point correlation functions are thus either holomorphic as in Eqs. (3.20a, 3.21a) or antiholomorphic as in Eqs. (3.20b, 3.21b).

Another important set of two-point correlation functions that we will need is

$$
\left\langle e^{+\mathrm{i} f \Phi_{\mu}(z, \bar{z})} e^{-\mathrm{i} f \Phi_{\nu}(0,0)}\right\rangle_{\Phi_{1}, \Phi_{2}} \propto \delta_{\mu \nu} z^{-2 h} \bar{z}^{-2 \bar{h}}, \quad \mu, \nu=1,2,
$$

where the conformal weights $h$ and $\bar{h}$ are given by 


$$
h=\bar{h}=\frac{f^{2}}{8 \pi} .
$$

The proportionality constant on the right-hand-side of Eq. (3.22a) depends on the short distance cutoff used to regulate the free scalar field theory. The parameter $f$ is analytically continued from the real numbers to arbitrary complex numbers. In particular, we will be interested in purely imaginary values of $f$ owing to the non-compact nature of the decoupling transformation in the longitudianal $\left(\Phi_{1}\right)$ vector gauge sector.

For any given Euclidean effective action $S_{\mathrm{cr}}^{(m, n)}$ in Eq. (3.14), we can calculate the scaling dimensions of all possible local operators, thus explicitly showing that all correlation functions are algebraic. To illustrate how this is done, we begin with the effective action $S_{\mathrm{cr}}^{(1,0)}$ for the averaged retarded single-particle Green function. One verifies that

$$
\begin{aligned}
& \left\langle\psi_{+}(z, \bar{z}) \psi_{+}^{\dagger}(0,0)\right\rangle=\left\langle\varphi_{+}(z, \bar{z}) \varphi_{+}^{\dagger}(0,0)\right\rangle=\frac{1}{2 \pi z} \\
& \left\langle\psi_{-}(z, \bar{z}) \psi_{-}^{\dagger}(0,0)\right\rangle=\left\langle\varphi_{-}(z, \bar{z}) \varphi_{-}^{\dagger}(0,0)\right\rangle=\frac{1}{2 \pi \bar{z}}
\end{aligned}
$$

as follows immediately from, say,

$$
\begin{aligned}
\left\langle\psi_{ \pm}^{\dagger}(z, \bar{z}) \psi_{ \pm}(0,0)\right\rangle & =\left\langle\psi_{ \pm}^{\prime \dagger}(z, \bar{z}) \psi_{ \pm}^{\prime}(0,0)\right\rangle \\
& \times\left\langle e^{\mathrm{i}\left( \pm \mathrm{i} \sqrt{g_{A}}\right) \Phi_{1}(z, \bar{z})} e^{\mathrm{i}\left(\mp \mathrm{i} \sqrt{g_{A}}\right) \Phi_{1}(0,0)}\right\rangle \\
& \times\left\langle e^{\mathrm{i}\left(-\sqrt{g_{A}}\right) \Phi_{2}(z, \bar{z})} e^{\mathrm{i}\left(+\sqrt{g_{A}}\right) \Phi_{2}(0,0)}\right\rangle .
\end{aligned}
$$

The bracket denotes an average performed with the effective action

$$
S_{\mathrm{cr}}^{(1,0)}=S_{\mathrm{imp}}\left[\Phi_{1}\right]+S_{\mathrm{imp}}\left[\Phi_{2}\right]-\mathrm{i} S_{\mathrm{gh}}-\mathrm{i} S_{\mathrm{r}}^{(1)} .
$$

We see that any impurity $\left(g_{A}\right)$ dependency coming from averaging over $\Phi_{1}$ precisely cancels that coming from averaging over $\Phi_{2}$. Hence the scaling properties of the fields $\psi_{ \pm}$and $\varphi_{ \pm}$are not affected by impurity averaging. This is not so, however, when calculating the two-point function for the composite operator

$$
\mathcal{O}(z, \bar{z})=\left(\psi_{+}^{\dagger m_{1}} \psi_{-}^{\dagger m_{2}} \psi_{+}^{m_{3}} \psi_{-}^{m_{4}} \varphi_{+}^{\dagger m_{5}} \varphi_{-}^{\dagger m_{6}} \varphi_{+}^{m_{7}} \varphi_{-}^{m_{8}}\right)(z, \bar{z}),
$$

which is defined through point-splitting $\left(m_{1}, \cdots, m_{8}\right.$ are arbitrary positive integers). Indeed,

$$
\begin{aligned}
\left\langle\mathcal{O}^{\dagger}(z, \bar{z}) \mathcal{O}(0,0)\right\rangle & \propto\left\langle\psi_{-}^{\prime \dagger m_{4}}(z, \bar{z}) \psi_{-}^{\prime m_{4}}(0,0)\right\rangle\left\langle\psi_{+}^{\prime \dagger m_{3}}(z, \bar{z}) \psi_{+}^{\prime m_{3}}(0,0)\right\rangle \\
& \times\left\langle\psi_{-}^{\prime m_{2}}(z, \bar{z}) \psi_{-}^{\prime \dagger m_{2}}(0,0)\right\rangle\left\langle\psi_{+}^{\prime m_{1}}(z, \bar{z}) \psi_{+}^{\prime \dagger m_{1}}(0,0)\right\rangle \\
& \times\left\langle\varphi_{-}^{\prime \dagger m_{8}}(z, \bar{z}) \varphi_{-}^{\prime m_{8}}(0,0)\right\rangle\left\langle\varphi_{+}^{\prime \dagger m_{7}}(z, \bar{z}) \varphi_{+}^{\prime m_{7}}(0,0)\right\rangle \\
& \times\left\langle\varphi_{-}^{\prime m_{6}}(z, \bar{z}) \varphi_{-}^{\prime \dagger m_{6}}(0,0)\right\rangle\left\langle\varphi_{+}^{\prime m_{5}}(z, \bar{z}) \varphi_{+}^{\prime \dagger m_{5}}(0,0)\right\rangle \\
& \times\left\langle e^{+\mathrm{i} f_{1} \sqrt{g_{A}} \Phi_{1}(z, \bar{z})} e^{-\mathrm{i} f_{1} \sqrt{g_{A}} \Phi_{1}(0,0)}\right\rangle \\
& \times\left\langle e^{+\mathrm{i} f_{2} \sqrt{g_{A}} \Phi_{2}(z, \bar{z})} e^{-\mathrm{i} f_{2} \sqrt{g_{A}} \Phi_{2}(0,0)}\right\rangle .
\end{aligned}
$$

The conformal weight $h(\bar{h})$ of $\psi_{+(-)}^{\prime m}$ scales quadratically with $m$ in contrast to that of $\varphi_{+(-)}^{\prime m}$ which scales linearly with $m$. This is perhaps most easily seen with the help of Abelian bosonization when calculating correlation functions of $\psi_{ \pm}^{\prime}$ 's. Exponentials in the longitudinal and transversal components of the random gauge fields scale as in Eq. (3.22) with

$$
\begin{aligned}
& f_{1}=-\mathrm{i}\left(m_{1}-m_{2}-m_{3}+m_{4}+m_{5}-m_{6}-m_{7}+m_{8}\right), \\
& f_{2}=+\left(m_{1}+m_{2}-m_{3}-m_{4}+m_{5}+m_{6}-m_{7}-m_{8}\right),
\end{aligned}
$$

respectively. Notice the difference in the relative sign of the $m$ 's in $f_{1}$ and $f_{2}$. We thus conclude 34 that $\mathcal{O}$ scales like 


$$
\left\langle\mathcal{O}^{\dagger}(z, \bar{z}) \mathcal{O}(0,0)\right\rangle_{S_{\mathrm{cr}}^{(1,0)}} \propto z^{-2 h} \bar{z}^{-2 \bar{h}}
$$

where the conformal weights are given by

$$
\begin{aligned}
& h=\frac{1}{2}\left[m_{1}^{2}+m_{3}^{2}+m_{5}+m_{7}+\left(f_{2}^{2}-\left|f_{1}\right|^{2}\right) \frac{g_{A}}{4 \pi}\right], \\
& \bar{h}=\frac{1}{2}\left[m_{2}^{2}+m_{4}^{2}+m_{6}+m_{8}+\left(f_{2}^{2}-\left|f_{1}\right|^{2}\right) \frac{g_{A}}{4 \pi}\right] .
\end{aligned}
$$

Impurity averaging does not change the scaling dimensions of local operators when $f_{2}^{2}=\left|f_{1}\right|^{2}$. On the other hand, the non-compactness of the decoupling transformation allows for the possibility that local operators have negative scaling dimensions when $\left|f_{1}\right|^{2} \gg f_{2}^{2}$.

Negative dimensional operators are now easily obtained in the theory with effective action $S_{\mathrm{cr}}^{(1,0)}$. For example, consider the composite operator $\Psi_{n_{1} n_{2}}$ defined by

$$
\Psi_{n_{1} n_{2}}= \begin{cases}\varphi_{+}^{n_{1}} \varphi_{-}^{n_{2}}, & n_{1}>0, n_{2}>0, \\ \varphi_{+}^{\dagger-n_{1}} \varphi_{-}^{n_{2}}, & n_{1}<0, n_{2}>0, \\ \varphi_{+}^{n_{1}} \varphi_{-}^{\dagger-n_{2}}, & n_{1}>0, n_{2}<0, \\ \varphi_{+}^{\dagger-n_{1}} \varphi_{-}^{\dagger-n_{2}}, & n_{1}<0, n_{2}<0,\end{cases}
$$

and use Eqs. (3.28-3.29). The conformal weights of these operators are

$$
h=\frac{1}{2}\left|n_{1}\right|+\frac{g_{A}}{2 \pi} n_{1} n_{2}, \quad \bar{h}=\frac{1}{2}\left|n_{2}\right|+\frac{g_{A}}{2 \pi} n_{1} n_{2} .
$$

This demonstrates that for any given value of $g_{A}$, there are infinitely many local composite operators with negative conformal weights. This is very different from unitary two-dimensional conformal field theory, say the $U(1)$ Thirring model [46] (the action for the Euclidean $U(1)$ Thirring model differs from $S_{\mathrm{cr}}^{(1,0)}$ only in that the $\varphi$ sector is absent), which cannot support operators with negative dimensions. Operators like $\Psi_{-|n||n|}$ are not mere curiosities. For one thing, they are generated in the effective graded supersymmetric model if non-Gaussian moments in the probability distribution of a mass-like random perturbation of the Dirac Hamiltonian are present. Furthermore, they yield direct information on the impurity average of products of single-particle Green functions as we now show.

The generalization to the calculation of the scaling dimension of any local operator in the theory with effective action $S_{\mathrm{cr}}^{(m, n)}$ is now obvious. The most general local composite operator

$$
\mathcal{O}(z, \bar{z})=\prod_{k=1}^{m+n}\left(\psi_{k+}^{\dagger m_{1 k}} \psi_{k-}^{\dagger m_{2 k}} \psi_{k+}^{m_{3 k}} \psi_{k-}^{m_{4 k}} \varphi_{k+}^{\dagger m_{5 k}} \varphi_{k-}^{\dagger m_{6 k}} \varphi_{k+}^{m_{7 k}} \varphi_{k-}^{m_{8 k}}\right)(z, \bar{z})
$$

where point-splitting is implied and all $m$ 's are positive integers, scales like

$$
\begin{aligned}
& \left\langle\mathcal{O}^{\dagger}(z, \bar{z}) \mathcal{O}(0,0)\right\rangle_{S_{\mathrm{cr}}^{(m, n)}} \propto z^{-2 h} \bar{z}^{-2 \bar{h}} \\
& h=\frac{1}{2} \sum_{k=1}^{m+n}\left[m_{1 k}^{2}+m_{3 k}^{2}+m_{5 k}+m_{7 k}\right]+\left(f_{2}^{2}-\left|f_{1}\right|^{2}\right) \frac{g_{A}}{8 \pi} \\
& \bar{h}=\frac{1}{2} \sum_{k=1}^{m+n}\left[m_{2 k}^{2}+m_{4 k}^{2}+m_{6 k}+m_{8 k}\right]+\left(f_{2}^{2}-\left|f_{1}\right|^{2}\right) \frac{g_{A}}{8 \pi} \\
& f_{1}=-\mathrm{i} \sum_{k=1}^{m+n}\left(m_{1 k}-m_{2 k}-m_{3 k}+m_{4 k}+m_{5 k}-m_{6 k}-m_{7 k}+m_{8 k}\right), \\
& f_{2}=+\sum_{k=1}^{m+n}\left(m_{1 k}+m_{2 k}-m_{3 k}-m_{4 k}+m_{5 k}+m_{6 k}-m_{7 k}-m_{8 k}\right) .
\end{aligned}
$$

An important consequence which follows from Eqs. 3.29 3.28) and Eq. (3.31) is that

$$
\left\langle\mathcal{O}^{\dagger}(z, \bar{z}) \mathcal{O}(0,0)\right\rangle_{S_{\mathrm{cr}}^{(1,0)}} \sim\left\langle\mathcal{P}^{\dagger}(z, \bar{z}) \mathcal{P}(0,0)\right\rangle_{S_{\mathrm{cr}}^{(m-j, j)}}, \quad j=0, \cdots, m,
$$


where

$$
\begin{aligned}
& \mathcal{O}(z, \bar{z})=\left(\varphi_{+}^{\dagger m_{5}} \varphi_{-}^{\dagger m_{6}} \varphi_{+}^{m_{7}} \varphi_{-}^{m_{8}}\right)(z, \bar{z}), \\
& \mathcal{P}(z, \bar{z})=\left(\prod_{i=1}^{m_{5}} \prod_{j=1}^{m_{6}} \prod_{k=1}^{m_{7}} \prod_{l=1}^{m_{8}} \varphi_{i+}^{\dagger} \varphi_{j-}^{\dagger} \varphi_{k+} \varphi_{l-}\right)(z, \bar{z}), \\
& m=m_{5}+m_{6}+m_{7}+m_{8} .
\end{aligned}
$$

Eq. (3.32a relates correlations in a model with several copies of the pair of spinors $(\psi, \varphi)$ to a model with a single pair. Eq. (3.32a) is only strictly true in the limit $z, \bar{z} \rightarrow 0$. There are subleading corrections to this algebraic decay, in particular when $j \neq 0, m$. It is an example of an operator product expansion. Eq. (3.32) allows to relate the operator content in the theory with effective action $S_{\mathrm{cr}}^{(1,0)}$ to impurity averaging of higher powers of the single-particle Green functions. For example, it tells us that the scaling dimension of $\Psi_{-m m}, m$ a positive integer, in the theory

with effective action $S_{\mathrm{cr}}^{(1,0)}$ is the same as the scaling dimension of the operator whose expectation value in the theory with effective action $S_{\mathrm{cr}}^{(m, 0)}$ yields the impurity average $\overline{[\operatorname{tr} G(x, x, 0 ; V)]^{m}}$. Indeed, we recall that

$$
\overline{[\operatorname{tr} G(x, x, 0 ; V)]^{m}} \propto\left\langle\prod_{k=1}^{m}\left[\psi_{k+} \psi_{k-}^{\dagger}+\psi_{k-} \psi_{k+}^{\dagger}+\varphi_{k+} \varphi_{k-}^{\dagger}+\varphi_{k-} \varphi_{k+}^{\dagger}\right](x)\right\rangle_{S_{\mathrm{cr}}^{(m, 0)}} .
$$

After expanding the product on the right-hand-side of Eq. (3.33), we can calculate the scaling dimensions of any of the resulting $4^{m}$ terms using Eq. (3.31). They all share the scaling dimension of

$$
\mathcal{P}(x)=\prod_{k=1}^{m}\left(\varphi_{k+} \varphi_{k-}^{\dagger}\right)(x)
$$

which in turn can be extracted from the algebraic decay of

$$
\left\langle\mathcal{P}^{\dagger}(x) \mathcal{P}(y)\right\rangle_{S_{\mathrm{cr}}^{(m, 0)}} \propto\left\langle\left(\varphi_{-} \varphi_{+}^{\dagger}\right)^{m}(x)\left(\varphi_{+} \varphi_{-}^{\dagger}\right)^{m}(y)\right\rangle_{S_{\mathrm{cr}}^{(1,0)}} .
$$

\section{NON-ABELIAN VECTOR GAUGE RANDOMNESS}

Tight-binding one-body Hamiltonians, for which the Fermi surface is made of a collection of isolated points in the Brillouin zone, are well described by, say, $N$ species of Dirac fermions, if one is only interested in the long wavelength and low energy properties of the system. In general, impurities induce one-body interactions among the $N$ different species. In this section, we consider such a two-dimensional system in which impurities can be represented by a static Abelian vector gauge-like interaction on the one hand, and by a static non-Abelian vector gauge-like interaction on the other hand. The Abelian vector gauge potential $A_{\mu}$ does not induce scattering events between different species. The non-Abelian vector gauge potential $B_{\mu}^{a}$ does. The index $\mu=1,2$ denotes the two spatial components of the gauge fields, and $a=1, \cdots, N^{2}-1$ labels any basis $\left\{T^{a}\right\}$ of traceless Hermitean $N \times N$ matrices. The Hamitonian of the pure system is the $2 N \times 2 N$ matrix

$$
H_{0}=-\mathrm{i} I \gamma_{\mu} \partial_{\mu}
$$

where $I$ is the $N \times N$ unit matrix. The impurity potential is given by the $2 N \times 2 N$ matrix

$$
V(x)=\gamma_{\mu}\left[A_{\mu}(x) I+B_{\mu}(x)\right]
$$

where

$$
B_{\mu}=B_{\mu}^{a} T^{a},
$$

transforms like the adjoint of the Lie algebra $s u(N)$. Our conventions for the generators of $s u(N)$ are

$$
\operatorname{tr}\left(T^{a} T^{b}\right)=\frac{\delta^{a b}}{2}, \quad\left[T^{a}, T^{b}\right]=\mathrm{i} f^{a b c} T^{c}, \quad f^{a c d} f^{b c d}=N \delta^{a b}, \quad a, b, c=1, \cdots, N^{2}-1 .
$$


Any impurity average over a product of single-particle Green functions can be obtained from an effective partition function. We are only interested in the single-particle Green functions in which all energies have been set to zero. Without loss of generality, we will concentrate on the averaged single-particle Green function. It can be obtained from the expectation value of $\psi(x) \bar{\psi}(y)+\varphi(x) \bar{\varphi}(y)$ with respect to the partition function

$$
\begin{aligned}
\mathcal{Z} & =\int \mathcal{D}[\bar{\psi}, \psi, \bar{\varphi}, \varphi] \int \mathcal{D}\left[A_{\mu}\right] \int \mathcal{D}\left[B_{\mu}\right] e^{-\int d^{2} x \mathcal{L}}, \\
\mathcal{L} & =\bar{\psi} \gamma_{\mu}\left(\partial_{\mu}+\mathrm{i} A_{\mu} I+\mathrm{i} B_{\mu}\right) \psi+\bar{\varphi} \gamma_{\mu}\left(\partial_{\mu}+\mathrm{i} A_{\mu} I+\mathrm{i} B_{\mu}\right) \varphi \\
& +\frac{1}{2 g_{A}} A_{\mu} A_{\mu}+\frac{1}{g_{B}} \operatorname{tr}\left(B_{\mu} B_{\mu}\right) .
\end{aligned}
$$

The Dirac spinor $\psi$ denotes a Grassmann coherent state, $\varphi$ a bosonic one. Both transform like the fundamental representation of $s u(N)$. The vector gauge fields are still dimensionfull, although they have been rescaled compared to section [II]. The positive impurity strengths $g_{A}$ and $g_{B}$ act like inverse masses for the Abelian and non-Abelian vector gauge fields, respectively. We show in this section that the partition function in Eq. (4.5a) factorizes into four independent sectors. However, in contrast to the case of Abelian vector gauge impurity only, we show that $\mathcal{Z}$ does not describe a theory at criticality for all but one value of $g_{B}>0$. Criticality is proved for the special case $g_{B}=\infty$. In this limit, $\mathcal{L}$ in Eq. $4.5 \mathrm{~b}$ ) is shown to be conformally invariant, and to yield an energy-momentum tensor with a vanishing central charge in its operator product expansion in agreement with Ref. [47]. The vanishing of the central charge follows from the fact that by construction $\mathcal{Z}=1$, and thus serves as a consistency check. The operator content of $\mathcal{Z}$ is constructed for $g_{A}=0, g_{B}=\infty$ and we obtain the new result that an infinite hierarchy of negative dimensional operators is present at the (non-Abelian) disordered critical point.

\section{A. Operator content at criticality for non-Abelian vector gauge randomness}

We begin by showing that it is possible to decouple the impurities from the spinor coherent states. To this end, let

$$
\begin{array}{ll}
A_{\bar{z}} \equiv A_{1}-\mathrm{i} A_{2}, & A_{z} \equiv A_{1}+\mathrm{i} A_{2}, \\
B_{\bar{z}} \equiv B_{1}-\mathrm{i} B_{2}, & B_{z} \equiv B_{1}+\mathrm{i} B_{2} .
\end{array}
$$

The antiholomorphic components $A_{\bar{z}}$ and $B_{\bar{z}}$ of the gauge fields are independent from the holomorphic components $A_{z}$ and $B_{z}$. Both antiholomorphic and holomorphic components of the gauge fields belong to the complex extensions $u^{c *}(N)$ and $u^{c}(N)$, respectively, of the real Lie Alebra $u(N)$. The antiholomorphic and holomorphic components are a convenient choice when working with the chiral basis for the spinors since

$$
\begin{aligned}
\mathcal{L} & =\psi_{+}^{\dagger}\left(2 \partial_{\bar{z}}+\mathrm{i} A_{\bar{z}} I+\mathrm{i} B_{\bar{z}}\right) \psi_{+}+\psi_{-}^{\dagger}\left(2 \partial_{z}+\mathrm{i} A_{z} I+\mathrm{i} B_{z}\right) \psi_{-} \\
& +\varphi_{+}^{\dagger}\left(2 \partial_{\bar{z}}+\mathrm{i} A_{\bar{z}} I+\mathrm{i} B_{\bar{z}}\right) \varphi_{+}+\varphi_{-}^{\dagger}\left(2 \partial_{z}+\mathrm{i} A_{z} I+\mathrm{i} B_{z}\right) \varphi_{-} \\
& +\frac{1}{2 g_{A}} A_{+} A_{-}+\frac{1}{g_{B}} \operatorname{tr}\left(B_{+} B_{-}\right) .
\end{aligned}
$$

We parametrize the components of the gauge fields in the Lie algebras $u^{c *}(N)$ and $u^{c}(N)$ by fields in the complex extensions $U^{c *}(N)$ and $U^{c}(N)$ of the Lie group $U(N)$ :

$$
\begin{array}{lll}
A_{\bar{z}} I=+\mathrm{i}\left(2 \partial_{\bar{z}} H_{\bar{z}}\right) H_{\bar{z}}^{-1}, & H_{\bar{z}}=e^{-\mathrm{i} I \phi_{\bar{z}}}, \quad \phi_{\bar{z}}=\Phi_{2}-\mathrm{i} \Phi_{1}, \\
A_{z} I \equiv+\mathrm{i}\left(2 \partial_{z} H_{z}\right) H_{z}^{-1}, & H_{z}=e^{-\mathrm{i} I \phi_{z}}, \quad \phi_{z}=\Phi_{2}+\mathrm{i} \Phi_{1}, \\
B_{\bar{z}} \equiv+\mathrm{i}\left(2 \partial_{\bar{z}} G_{\bar{z}}\right) G_{\bar{z}}^{-1}, & G_{\bar{z}} \in S U^{c *}(N), \\
B_{z} \equiv+\mathrm{i}\left(2 \partial_{z} G_{z}\right) G_{z}^{-1}, & G_{z} \in S U^{c}(N) .
\end{array}
$$

This change of variables costs the Jacobian

$$
\mathcal{J}_{\text {gh }}=\operatorname{Det}\left(2 \partial_{\bar{z}}\right) \operatorname{Det}\left(2 \partial_{z}\right) \operatorname{Det}\left(\nabla_{\bar{z}}\right) \operatorname{Det}\left(\nabla_{z}\right) \text {, }
$$

where $\nabla_{\bar{z}}$ and $\nabla_{z}$ are the covariant derivatives in the adjoint representations of $S U^{c *}(N)$ and $S U^{c}(N)$, respectively. For example

$$
\begin{aligned}
\delta A_{\bar{z}} & =\mathrm{i} \delta\left(\left(2 \partial_{\bar{z}} G_{\bar{z}}\right) G_{\bar{z}}^{-1}\right) \\
& =\left\{2 \partial_{\bar{z}} \cdot+\mathrm{i}\left[\mathrm{i}\left(2 \partial_{\bar{z}} G_{\bar{z}}\right) G_{\bar{z}}^{-1}, \cdot\right]\right\}\left(\mathrm{i}\left(\delta G_{\bar{z}}\right) G_{\bar{z}}^{-1}\right) .
\end{aligned}
$$


We represent this Jacobian as a path integral over auxiliary Grassmann fields (ghosts):

$$
\begin{aligned}
\operatorname{Det}\left(2 \partial_{\bar{z}}\right) & =\int \mathcal{D}\left[\beta_{+}^{0}, \alpha_{+}^{0}\right] e^{+\mathrm{i} \int d^{2} x 2 \operatorname{tr}\left(\beta_{+}^{0} T^{0} \mathrm{i} 2 \partial_{\bar{z}} T^{0} \alpha_{+}^{0}\right)}, \\
\operatorname{Det}\left(2 \partial_{z}\right) & =\int \mathcal{D}\left[\beta_{-}^{0}, \alpha_{-}^{0}\right] e^{+\mathrm{i} \int d^{2} x 2 \operatorname{tr}\left(\beta_{-}^{0} T^{0} \mathrm{i} 2 \partial_{z} T^{0} \alpha_{-}^{0}\right)}, \\
\operatorname{Det}\left(\nabla_{\bar{z}}\right) & =\int \mathcal{D}\left[\beta_{+}^{a}, \alpha_{+}^{a}\right] e^{+\mathrm{i} \int d^{2} x 2 \operatorname{tr}\left(\beta_{+}^{a} T^{a} \mathrm{i} \nabla_{\bar{z}} T^{b} \alpha_{+}^{b}\right)}, \\
\operatorname{Det}\left(\nabla_{z}\right) & =\int \mathcal{D}\left[\beta_{-}^{a}, \alpha_{-}^{a}\right] e^{+\mathrm{i} \int d^{2} x 2 \operatorname{tr}\left(\beta_{-}^{a} T^{a} \mathrm{i} \nabla_{z} T^{b} \alpha_{-}^{b}\right)} .
\end{aligned}
$$

The unit matrix $I$ has been rescaled to $T^{0} \equiv \frac{1}{\sqrt{2 N}} I$ for notational convenience.

We are now ready to decouple the vector gauge fields from all other fields. For example, the chiral transformation

$$
\begin{array}{ll}
\psi_{+}^{\dagger}=\psi_{+}^{\prime \dagger} G_{\bar{z}}^{-1} H_{\bar{z}}^{-1}, & \psi_{+}=H_{\bar{z}} G_{\bar{z}} \psi_{+}^{\prime}, \\
\psi_{-}^{\dagger}=\psi_{-}^{\prime \dagger} G_{z}^{-1} H_{z}^{-1}, & \psi_{-}=H_{z} G_{z} \psi_{-}^{\prime},
\end{array}
$$

decouples the $\psi$ sector from the vector gauge fields at the price of the Jacobian 44

$$
\mathcal{J}_{\psi}=\frac{\operatorname{Det}\left[\mathrm{i} \gamma_{\mu}\left(\partial_{\mu}+\mathrm{i} A_{\mu} I\right)\right]}{\operatorname{Det}\left(\mathrm{i} \gamma_{\mu} \partial_{\mu}\right)} \times \frac{\operatorname{Det}\left[\mathrm{i} \gamma_{\mu}\left(\partial_{\mu}+\mathrm{i} B_{\mu}\right)\right]}{\operatorname{Det}\left(\mathrm{i} \gamma_{\mu} \partial_{\mu}\right)} \text {. }
$$

Similarly, the chiral transformation

$$
\begin{aligned}
\beta_{+}^{a} T^{a} & =G_{\bar{z}} \beta_{+}^{\prime a} T^{a} G_{\bar{z}}^{-1}, & \alpha_{+}^{a} T^{a} & =G_{\bar{z}} \alpha_{+}^{\prime a} T^{a} G_{\bar{z}}^{-1}, \\
\beta_{-}^{a} T^{a} & =G_{z} \beta_{-}^{\prime a} T^{a} G_{z}^{-1}, & \alpha_{-}^{a} T^{a} & =G_{z} \alpha_{-}^{\prime a} T^{a} G_{z}^{-1},
\end{aligned}
$$

decouples the ghosts from the vector gauge fields at the price of the Jacobian [48]

$$
\mathcal{J}_{\alpha^{1}, \cdots, \alpha^{N}}=\left\{\frac{\operatorname{Det}\left[\mathrm{i} \gamma_{\mu}\left(\partial_{\mu}+\mathrm{i} B_{\mu}\right)\right]}{\operatorname{Det}\left(\mathrm{i} \gamma_{\mu} \partial_{\mu}\right)}\right\}^{2 N} .
$$

The exponent $2 N$ is twice the quadratic Casimir eigenvalue $c_{v}=N$ in the adjoint representation as is apparent from Eq. (4.4). Finally, by performing exactly the same chiral transformation on the $\varphi$ 's as we did on the $\psi$ 's, we can entirely decouple the spinor sector from the vector gauge sector. Decoupling the $\varphi$ 's from the gauge fields costs a Jacobian

$$
\mathcal{J}_{\varphi}=\mathcal{J}_{\psi}^{-1}
$$

which cancels the one coming from the $\psi$ sector. We are then left with the Jacobian $\mathcal{J}_{\alpha^{1}, \ldots, \alpha^{N}}$ from the ghost sector.

Polyakov and Wiegmann have calculated $\mathcal{J}_{\alpha^{1}, \cdots, \alpha^{N}}$. It is given by the exponential of the Euclidean Wess-ZuminoWitten action 49:

$$
\mathcal{J}_{\alpha^{1}, \cdots, \alpha^{N}}=e^{-(-2 N) \Gamma\left[G_{z}^{-1} G_{\bar{z}}\right]},
$$

where the Euclidean Wess-Zumino-Witten action is given by

$$
\begin{aligned}
\Gamma[G] & =\frac{1}{8 \pi} \int d x_{1} d x_{2} \operatorname{tr}\left[\left(\partial_{\mu} G\right)\left(\partial_{\mu} G^{-1}\right)\right] \\
& -\frac{\mathrm{i}}{12 \pi} \int_{\mathrm{B}, \partial \mathrm{B}=\mathrm{S}^{2}} d x_{1} d x_{2} d x_{3} \epsilon_{\mu \nu \lambda} \operatorname{tr}\left[\left(\partial_{\mu} G\right) G^{-1}\left(\partial_{\nu} G\right) G^{-1}\left(\partial_{\lambda} G\right) G^{-1}\right] .
\end{aligned}
$$

Before collecting all contributions to the effective Lagrangian density, we rewrite the probability distributions for the vector gauge fields in a more useful form. In the Abelian sector, the Gaussian weight is

$$
+\frac{1}{2 g_{A}} A_{\bar{z}} A_{z}=-\frac{1}{2 g_{A} N} \operatorname{tr}\left[\left(2 \partial_{\bar{z}} H_{\bar{z}}\right) H_{\bar{z}}^{-1}\left(2 \partial_{z} H_{z}\right) H_{z}^{-1}\right] .
$$


In the non-Abelian sector, the Gaussian weight is

$$
+\frac{1}{g_{B}} \operatorname{tr}\left(B_{\bar{z}} B_{z}\right)=-\frac{1}{g_{B}} \operatorname{tr}\left[\left(2 \partial_{\bar{z}} G_{\bar{z}}\right) G_{\bar{z}}^{-1}\left(2 \partial_{z} G_{z}\right) G_{z}^{-1}\right] .
$$

With the help of the Polyakov-Wiegmann identity 50

$$
\Gamma\left[G^{-1} H\right]=\Gamma\left[G^{-1}\right]+\Gamma[H]+\frac{1}{4 \pi} \int d x_{1} d x_{2} \operatorname{tr}\left[\left(2 \partial_{z} G\right) G^{-1}\left(2 \partial_{\bar{z}} H\right) H^{-1}\right]
$$

the Gaussian weights for the Abelian and non-Abelian vector gauge fields are rewritten

$$
+\frac{1}{2 g_{A}} A_{\bar{z}} A_{z}=-\frac{\pi}{g_{A} N}\left(\Gamma\left[H_{z}^{-1} H_{\bar{z}}\right]-\Gamma\left[H_{z} H_{\bar{z}}\right]\right)
$$

and

$$
+\frac{1}{g_{B}} \operatorname{tr}\left(B_{\bar{z}} B_{z}\right)=-\frac{4 \pi}{g_{B}}\left(\Gamma\left[G_{z}^{-1} G_{\bar{z}}\right]-\Gamma\left[G_{z}^{-1}\right]-\Gamma\left[G_{\bar{z}}\right]\right)
$$

respectively. In the Abelian case, the mass term separates into two independent sectors. In the non-Abelian case, due to the topological term in the Wess-Zumino-Witten action, the mass term does not separate into two independent sectors.

We conclude that the trace of the averaged single-particle Green function in which all energies have been set to zero can be obtained from the expectation value of

$$
\begin{aligned}
& \operatorname{tr}[\psi(x) \bar{\psi}(y)+\varphi(x) \bar{\varphi}(y)]= \\
& \operatorname{tr}\left[\left(H_{\bar{z}} G_{\bar{z}} \psi_{+}^{\prime}\right)(x)\left(\psi_{-}^{\prime \dagger} G_{z}^{-1} H_{z}^{-1}\right)(y)+\left(H_{z} G_{z} \psi_{-}^{\prime}\right)(x)\left(\psi_{+}^{\prime \dagger} G_{\bar{z}}^{-1} H_{\bar{z}}^{-1}\right)(y)\right]+ \\
& \operatorname{tr}\left[\left(H_{\bar{z}} G_{\bar{z}} \varphi_{+}^{\prime}\right)(x)\left(\varphi_{-}^{\prime \dagger} G_{z}^{-1} H_{z}^{-1}\right)(y)+\left(H_{z} G_{z} \varphi_{-}^{\prime}\right)(x)\left(\varphi_{+}^{\prime \dagger} G_{\bar{z}}^{-1} H_{\bar{z}}^{-1}\right)(y)\right],
\end{aligned}
$$

with respect to the partition function

$$
\begin{aligned}
\mathcal{Z} & =\int \mathcal{D}\left[\psi_{ \pm}^{\prime \dagger}, \psi_{ \pm}^{\prime}, \varphi_{ \pm}^{\prime \dagger}, \varphi_{ \pm}^{\prime}\right] \int \mathcal{D}\left[\beta_{ \pm}^{\prime a}, \alpha_{ \pm}^{\prime a}\right] \int \mathcal{D}\left[H_{\bar{z}}, H_{z}\right] \int \mathcal{D}\left[G_{\bar{z}}, G_{z}\right] e^{-\left(S_{1}+S_{2}+S_{3}+S_{4}\right)} \\
S_{1} & =\int d^{2} x\left(\psi_{+}^{\prime \dagger} 2 \partial_{\bar{z}} \psi_{+}^{\prime}+\psi_{-}^{\prime \dagger} 2 \partial_{z} \psi_{-}^{\prime}+\varphi_{+}^{\prime \dagger} 2 \partial_{\bar{z}} \varphi_{+}^{\prime}+\varphi_{-}^{\prime \dagger} 2 \partial_{z} \varphi_{-}^{\prime}\right), \\
S_{2} & =2 \sum_{a, b=0}^{N} \int d^{2} x \operatorname{tr}\left(\beta_{+}^{\prime a} T^{a} 2 \partial_{\bar{z}} \alpha_{+}^{\prime b} T^{b}+\beta_{-}^{\prime a} T^{a} 2 \partial_{z} \alpha_{-}^{\prime b} T^{b}\right), \\
S_{3} & =-\frac{\pi}{g_{A} N} \Gamma\left[H_{z}^{-1} H_{\bar{z}}\right]+\frac{\pi}{g_{A} N} \Gamma\left[H_{z} H_{\bar{z}}\right], \\
S_{4} & =-\left[\frac{4 \pi}{g_{B}}+2 N\right] \Gamma\left[G_{z}^{-1} G_{\bar{z}}\right]+\frac{4 \pi}{g_{B}} \Gamma\left[G_{z}^{-1}\right]+\frac{4 \pi}{g_{B}} \Gamma\left[G_{\bar{z}}\right] .
\end{aligned}
$$

We can use the invariance of the Haar measure of $U^{c *}(N)\left(U^{c}(N)\right)$ under left and right multiplication to reparametrize the Abelian vector gauge components $H_{\bar{z}}$ and $H_{z}$ as $H_{z}^{-1} H_{\bar{z}}$ and $H_{z} H_{\bar{z}}$. The partition function has factorized into four independent sectors. The same factorization takes place for partition functions representing the impurity average over an arbitrary product of single-particle Green functions, as long as all energies in the Green functions are set to zero. However, the factorization property does not guaranty criticality, i.e., algebraic decay of all correlation functions.

The first sector describes a free massless relativistic theory with $U(N / N)$ graded symmetry. All Dirac spinors transform like the fundamental representation of $U(N)$. This theory is conformally invariant with primary fields $\psi_{ \pm}$ and $\varphi_{ \pm}$carrying the conformal weights

$$
(h, \bar{h})=\left(\frac{1}{2}, 0\right) \text { or }\left(0, \frac{1}{2}\right)
$$

depending on their chirality. The first sector has vanishing central charge 


$$
c_{1}=0
$$

since the partition function restricted to this sector is unity.

The second sector describes free massless relativistic ghosts transforming like the adjoint of $U(N)$. This theory has a conformally invariant energy-momentum tensor. The operator product expansion of the energy-momentum components yields the negative central charge [48]

$$
c_{2}=-2 \operatorname{dim} U(N)=-2 N^{2} .
$$

The third sector describes two independent and free scalar field theories and is thus conformally invariant. One has negative definite kinetic energy. Consequently, its primary field $H_{z}^{-1} H_{\bar{z}} \propto e^{\mathrm{i}\left(2 \mathrm{i} \Phi_{1}\right) I}$ carries negative conformal weights

$$
h=\bar{h}=-\frac{g_{A}}{8 \pi} .
$$

The scalar field theory with negative definite kinetic energy corresponds to the longitudinal component of the Abelian vector gauge field. The second scalar field theory has the primary field $H_{z} H_{\bar{z}} \propto e^{2 \mathrm{i} \Phi_{2} I}$ with positive conformal weight

$$
h=\bar{h}=+\frac{g_{A}}{8 \pi} .
$$

It corresponds to the transversal component of the Abelian vector gauge field. The central charge in the third sector is

$$
c_{3}=2
$$

The last sector describes the non-Abelian vector gauge fields. In contrast to the Abelian vector gauge sector, it is not conformally invariant for all values of the non-Abelian impurity strangth $g_{B}$. Only the limit $g_{B} \rightarrow \infty \beta^{3}$ yields a conformally invariant theory, namely the $S U(N)_{k}$ Wess-Zumino-Witten theory where $k=-2 N$ is the level of the underlying Kac-Moody algebra [51. The primary field $G_{z}^{-1} G_{\bar{z}}$ carries the conformal weight [51]

$$
h=\bar{h}=\frac{1}{N+k} \frac{N^{2}-1}{2 N}, \quad k=-2 N,
$$

which is thus negative. The associated central charge [51]

$$
c_{S U(N)_{k}}=\frac{k}{k+N}\left(N^{2}-1\right), \quad k=-2 N
$$

is positive. In the limit $g_{B} \rightarrow \infty$, there exists a local non-Abelian gauge symmetry with $G_{z} G_{\bar{z}}$ a pure gauge [52]. Integration over the pure gauge enforces the local constraint

$$
J_{\mu}^{a}=0, \quad \mu=1,2, \quad a=1, \cdots, N^{2}-1
$$

for the non-Abelian currents

$$
J_{\mu}^{a}=\bar{\psi} T^{a} \gamma_{\mu} \psi+\bar{\varphi} T^{a} \gamma_{\mu} \varphi, \quad \mu=1,2, \quad a=1, \cdots, N^{2}-1
$$

Thus, only $S U(N)$ gauge singlets can acquire an expectation value in this limit.

For all correlation functions calculated with the partition function in Eq. (4.5a) to be algebraic, one must take the limit $g_{B} \rightarrow \infty$. In this limit, the existence of a local gauge symmetry requires a careful definition of the measure of the vector gauge fields. Gauge fixing is in fact implicit in the chiral transformation which decouples the non-Abelian vector gauge fields from the spinors and ghosts. Indeed, one should think of the chiral transformation as the product of a pure gauge transformation, which amounts to gauge fixing, and of an axial transformation to achieve complete decoupling [52]. After gauge fixing, only gauge inequivalent configurations must be summed over in the partition function. Hence, the partition function restricted to the non-Abelian vector gauge sector is given by

\footnotetext{
${ }^{3}$ It is amusing to note that for negative values (and thus unphysical) of the non-Abelian impurity strength $g_{B}=-\frac{2 \pi}{N}$, the non-abelian sector is also "critical".
} 


$$
\mathcal{Z}_{4}^{\infty}=\int \mathcal{D}\left[G^{\prime}\right] e^{-(-2 N) \Gamma\left[G^{\prime}\right]}
$$

in the limit $g_{B} \rightarrow \infty$. To verify the correctness of Eq. (4.36), we calculate the total central charge:

$$
c=c_{1}+c_{2}+c_{3}+c_{S U(N)_{-2 N}}=0
$$

It vanishes as it should be.

The conformal weights of all primary field $\left\{\phi_{l}\right\}$ of the $S U(N)_{k}$ Wess-Zumino Witten theory have been calculated by Knizhnik and Zamoklodchikov [51]. They are given by

$$
h=\frac{c_{l}}{c_{v}+k}, \quad \bar{h}=\frac{c_{\bar{l}}}{c_{v}+k} .
$$

In the numerator, $c_{l}\left(c_{\bar{l}}\right)$ stands for the quadratic Casimir eigenvalue of the irreducible representation $l(\bar{l})$. Here, the representation $l(\bar{l})$ is defined by the transformation law obeyed by $\phi_{l}$ under $S U(N)$ rotations generated by the holomorphic (antiholomorphic) component of the level $k$ Kac-Moody currents. Thus, by analytical continuation of $k$ to the value $k=-2 N$, we see that all primary fields originating from the non-Abelian vector gauge impurity in the limit $g_{B}=\infty$ have negative conformal weights

$$
h=-\frac{c_{l}}{N}, \quad \bar{h}=-\frac{c_{\bar{l}}}{N} .
$$

The eigenvalues $\left\{c_{l}\right\}$ are known. Examples are

$$
c_{\mathrm{A}}=\frac{(N-2)(N+1)}{N}, \quad c_{\mathrm{fun}}=\frac{N^{2}-1}{2 N}, \quad c_{\mathrm{ad}}=N, \quad c_{\mathrm{S}}=\frac{(N+2)(N-1)}{N},
$$

in the antisymmetric $\square$, fundamental $\square$, adjoint, and symmetric $\square \square$ representations, respectively. Here, $\square$ represents a box in a Young tableau. We recall that all irreducible representations of $U(N)$ and $S U(N)$ can be labelled by Young tableaus made of $N$ rows with $f_{i}$ boxes in the $i$-th row, provided $f_{1}>f_{2}>\cdots>f_{N-1}>f_{N} \geq 0$. For $S U(N)$, $f_{N}$ always vanishes. It is known [53] that the $S U(N)$ irreducible representation with Young tableau $\left\{f_{i}\right\}$ made of $f=\sum_{i=1}^{N} f_{i}$ boxes, has the quadratic Casimir eigenvalue

$$
c_{\left\{f_{i}\right\}}=\frac{1}{2} \sum_{1=1}^{N}\left[f_{i}^{2}+(N+1-2 i) f_{i}\right]-\frac{f^{2}}{2 N} .
$$

We are now ready to compute the most relevant scaling dimensions controlling the expectation value of the local density operator $\bar{\psi} \psi+\bar{\varphi} \varphi$ and of higher powers thereof in the limit $g_{A}=0, g_{B} \rightarrow \infty$. Without loss of generality, we only need to consider powers of $\varphi_{-}^{\dagger} \varphi_{+}$. First and in agreement with Refs. 25, 47, the conformal weights of

$$
\left(\varphi_{-}^{\dagger} \varphi_{+}\right)(z, \bar{z})=\left(\varphi_{-}^{\prime \dagger} G^{\prime} \varphi_{+}^{\prime}\right)(z, \bar{z})
$$

are given by the quadratic Casimir operator in the fundamental representation $\square$ of $S U(N)$ :

$$
h=\bar{h}=\left(\frac{1}{2}+\frac{1}{N+k} \frac{N^{2}-1}{2 N}\right)_{k=-2 N}=\frac{1}{2 N^{2}} .
$$

Next, higher powers of $\varphi_{-}^{\dagger} \varphi_{+}$are defined through point-splitting:

$$
\left(\varphi_{-}^{\dagger} \varphi_{+}\right)^{m}(z, \bar{z})=\lim _{\substack{z_{i} \rightarrow z \\ \bar{z}_{i} \rightarrow \bar{z}}} \prod_{i=1}^{m}\left(\varphi_{-}^{\dagger} \varphi_{+}\right)\left(z_{i}, \bar{z}_{i}\right)=\lim _{\substack{z_{i} \rightarrow z \\ \bar{z}_{i} \rightarrow \bar{z}}} \prod_{i=1}^{m}\left(\varphi_{-}^{\prime \dagger} G^{\prime} \varphi_{+}^{\prime}\right)\left(z_{i}, \bar{z}_{i}\right) .
$$

We have already dealt with the product of $\varphi^{\prime}$ 's in section III A. The operator product expansion in the Wess-ZuminoWitten sector can be used to replace the point-splitted product of $G^{\prime}$ 's by a sum over primary fields $\phi_{l}(z, \bar{z})$. Each $\phi_{l}(z, \bar{z})$ in the sum corresponds to an irreducible representation present in the irreducible decomposition of the product representation $\square \otimes \cdots \otimes \square=\square \cdots \square \oplus \cdots$. It is thus the primary field $\phi_{m}$ associated to the irreducible representation 
with the largest quadratic Casimir eigenvalues $c_{m}$ and $c_{\bar{m}}$ in the irreducible decomposition of $\square \otimes \cdots \otimes \square$ which determines the conformal weights up to subleading corrections:

$$
h=\frac{m}{2}-\frac{c_{m}}{N}, \quad \bar{h}=\frac{m}{2}-\frac{c_{\bar{m}}}{N} .
$$

A first example is 47

$$
\begin{aligned}
& \left\langle\left(\varphi_{-}^{\dagger} \varphi_{+}\right)^{2}(z, \bar{z})\left(\varphi_{+}^{\dagger} \varphi_{-}\right)^{2}(0,0)\right\rangle \propto z^{-2 h} \bar{z}^{-2 \bar{h}}, \\
& h=\bar{h}=\left(1+\frac{(N+2)(N-1)}{N(N+k)}\right)_{k=-2 N}=-\frac{N-2}{N^{2}} .
\end{aligned}
$$

The conformal weights are those of the primary field transforming like the symmetric representation $\square \square$. They vanish for $S U(2)$ and are negative when $N>2$. Another example of a composite local operator with vanishing conformal weights is 47

$$
\begin{aligned}
& \left\langle\left(\varphi_{-}^{\dagger} \varphi_{+} \varphi_{+}^{\dagger} \varphi_{-}\right)(z, \bar{z})\left(\varphi_{-}^{\dagger} \varphi_{+} \varphi_{+}^{\dagger} \varphi_{-}\right)(0,0)\right\rangle \propto z^{-2 h} \bar{z}^{-2 \bar{h}}, \\
& h=\bar{h}=\left(1+\frac{N}{N+k}\right)_{k=-2 N}=0 .
\end{aligned}
$$

The conformal weights are those of the primary field transforming like the adjoint representation. One readily sees from Eq. (4.41) that the completely symmetric representation $\square \cdots \square=\{m, 0 \cdots, 0\}$, which corresponds to one row of $m$ boxes, has the largest quadratic Casimir eigenvalue among all irreducible representations made of $m$ boxes (for example, for $S U(2)$ it would correspond to the representation with largest possible "total spin" $\frac{m}{2}$ ). Hence, we find the important result

$$
\begin{aligned}
& \left\langle\left(\varphi_{-}^{\dagger} \varphi_{+}\right)^{m}(z, \bar{z})\left(\varphi_{+}^{\dagger} \varphi_{-}\right)^{m}(0,0)\right\rangle \propto z^{-2 h} \bar{z}^{-2 \bar{h}}, \\
& h=\bar{h}=\frac{m}{2}-\frac{N-1}{2 N^{2}}\left(m^{2}+N m\right) .
\end{aligned}
$$

As was the case along the critical line $g_{A}>0, g_{B}=0$, there are infinitely many local composite operators with negative scaling dimensions along the critical line $g_{A}=0, g_{B}=\infty$.

\section{SCALING AWAY FROM CRITICALITY}

In this section, we are going to calculate how some averaged quantities such as the local density of states, generalized inverse participation ratios, and their spatial correlations scale with respect to energy and length. We begin with the dynamical scaling exponent and with the local density of states.

\section{A. Dynamical scaling exponent $z$ and scaling of $\rho(\omega)$ away from criticality}

The dynamical scaling exponent $z$ relates scaling with energy to scaling with respect to length. We consider the local order parameter $\mathcal{M}(x)$ defined by

$$
\mathcal{M}(x) \equiv(\bar{\psi} \psi+\bar{\varphi} \varphi)(x)
$$

since it is coupled to the complex energy $E_{ \pm}=\omega \pm \mathrm{i} \eta$ in the retarded and advanced single-particle Green functions, respectively. Notice that $\mathcal{M}(x)$ breaks the global $U(1 / 1) \times U(1 / 1)$ invariance. We continue working in real space and choose a renormalization group scheme defined by the scale transformation

$$
\tilde{x}=a x, \quad a>0 .
$$

The scaling dimension of $E$ or, for that matter, those of $\psi, \varphi, A_{\mu}, B_{\mu}$, are defined by the requirement that the effective action in terms of the rescaled coordinates, fields, and coupling constants has the same form as that in terms of the original coordinates, fields, and coupling constants. The scaling dimensions of all local fields have been calculated 
in sections III and IV along the disordered critical line $g_{A} \geq 0, g_{B}=\infty$. The impurity strengths $g_{A}$ and $g_{B}$ are left unchanged by Eq. (5.2), since they couple conserved currents to vector gauge potentials. The energy scale $E$ transforms like

$$
\tilde{E} \equiv a^{-z} E, \quad z=1+\frac{g_{A}}{\pi}+\frac{N^{2}-1}{N^{2}}
$$

along the critical line $g_{A} \geq 0, g_{B}=\infty$, as follows from Eqs. (4.43) and (3.30b) and

$$
\begin{aligned}
& \tilde{E} \int d^{2} \tilde{x}\left(\tilde{\varphi}_{+}^{\dagger} \tilde{\varphi}_{-}\right)(\tilde{x})=\tilde{E} a^{2-(h+\bar{h})} \int d^{2} x\left(\varphi_{+}^{\dagger} \varphi_{-}\right)(x), \\
& h+\bar{h}=1-\frac{g_{A}}{\pi}-\frac{N^{2}-1}{N^{2}} \equiv 2-z .
\end{aligned}
$$

The dynamical exponent $z$ allows to turn scaling with respect to length into scaling with respect to energy. When only Abelian vector gauge disorder is present, $z=1+\frac{g_{A}}{\pi}$ is always larger than one and increases linearly with the impurity strength. It reaches the value $z=2$ when $g_{A}=\pi$. At this value, the scaling dimension of the local symmetry breaking field $\mathcal{M}(x)$ vanishes, a signal of spontaneous symmetry breaking. The possibility of spontaneous symmetry breaking cannot be ruled out by the Mermin-Wagner theorem as would be the case in two-dimensional unitary conformal field theory, since the disordered critical theory is not unitary. On the other hand, in the presence of non-Abelian vector gauge disorder only, the dynamical exponent $z=2-\frac{1}{N^{2}}$ is always smaller than two. Hence, the large impurity strength limit appears to be very different depending on whether the vector gauge impurity is Abelian or non-Abelian.

We now perturb the effective action $S_{\mathrm{cr}}^{(1,0)}$ at the critical point $g_{A} \geq 0, g_{B}=\infty$ with $\omega \int d^{2} x \mathcal{M}(x)$. To lowest order in $\omega$, the averaged local density of states $\rho(\omega)$ scales like

$$
\rho(\omega) \propto|\omega|^{\beta}, \quad \beta=\frac{2-z}{z}+\mathcal{O}(\omega),
$$

since, according to Eq. (5.4b), the scaling dimension of $\rho(\omega)$ is $2-z$. We see that in the presence of Abelian vector gauge-like disorder only, the averaged local density of states is finite at criticality for the impurity strength $g_{A}=\pi$. For larger values of $g_{A}, \rho(\omega)$ diverges in the limit $\omega \rightarrow 0$. In the presence of non-Abelian vector gauge disorder only, the scaling exponent $\beta=\left(2 N^{2}-1\right)^{-1}$ of the averaged local density of states decreases monotonically as a function of $N$ with the values $\beta=\frac{1}{7}$ when $N=2$ and $\beta=0$ when $N \rightarrow \infty$.

If the system is not in the thermodynamic limit but in a finite volume $L^{2}$, we recover Eq. (5.5) with the Ansatz

$$
\rho(\omega, L)=F_{\beta}\left(\omega L^{z}\right)|\omega|^{\beta}, \quad \omega \ll L^{-z},
$$

provided

$$
\lim _{x \rightarrow \infty} F_{\beta}(x)=K_{\beta}, \quad 0 \leq K_{\beta}<\infty
$$

holds. In a finite volume, the total number of energy eigenstates must be finite. This is always so (except in the limit $\left.g_{A} \rightarrow \infty\right)$, since $\beta>-1$.

\section{B. Scaling of averaged generalized participation ratios}

In this subsection, we turn our attention to the scaling of averaged generalized inverse participation ratios for three reasons. First, the scaling of averaged generalized inverse participation ratios was calculated perturbatively in powers of $\epsilon$, where space is $2+\epsilon$-dimensional, by Wegner for the non-linear $\sigma$ model of the metal-insulator transition at the mobility edge 112,14]. We will obtain non-perturbatively the dominant scaling exponents of averaged generalized inverse participation ratios for Dirac fermions with vector potential disorder. Second, we will see that the negative dimensional operators found in sections III and IV control the scaling of averaged generalized inverse participation ratios. Thus, negative dimensional operators are not mere curiosities of the disordered critical line $g_{A}>0, g_{B}=\infty$. Finally, we reexamine arguments suggesting a relationship between the scaling exponents of averaged generalized inverse participation ratios and the existence of a typical multifractal wave function with vanishing eigenenergy (critical wave function) 15. 
The concept of the inverse participation ratio for the states of a disordered system was introduced to distinguish localized from extended states $[54$. Let $|\Psi\rangle$ be any state defined in the Hilbert space representing a single-particle tightbinding Hamiltonian. The Hamiltonian describes, say, hopping on a finite $d$-dimensional lattice $\Lambda$ in the presence of a random site potential (Anderson model). There are $|\Lambda|$ sites labelled by $l=1, \cdots,|\Lambda|<\infty$. All states are normalized to one:

$$
\sum_{l \in \Lambda}|\langle l \mid \Psi\rangle|^{2}=\sum_{l \in \operatorname{supp} \Psi}|\langle l \mid \Psi\rangle|^{2}=1
$$

Here, supp $\Psi$ denotes all sites having a non-vanishing overlap with $|\Psi\rangle$. The inverse participation ratio is the functional

$$
\mathrm{P}_{\Lambda}^{(2)}[\Psi] \equiv \sum_{l \in \operatorname{supp} \Psi}|\langle l \mid \Psi\rangle|^{4}
$$

taking values in $] 0,1]$. Let now $\Omega$ be any subset of $\Lambda$ and define the normalized state $\left|\Psi_{\Omega}\right\rangle$ by

$$
\left\langle l \mid \Psi_{\Omega}\right\rangle= \begin{cases}|\Omega|^{-\frac{1}{2}}, & \forall l \in \Omega \\ 0, & \text { otherwise }\end{cases}
$$

On the one hand, the inverse participation ratio for the state $\left|\Psi_{\Lambda}\right\rangle$ scales with the linear size $L=|\Lambda|^{\frac{1}{d}}$ as

$$
\mathrm{P}_{\Lambda}^{(2)}\left[\Psi_{\Lambda}\right]=L^{-\tau(2)}, \quad \tau(2)=d
$$

On the other hand, the inverse participation ratio for the state $\left|\Psi_{\Omega}\right\rangle$, where $\Omega$ has a finite number of sites in the thermodynamic limit $L \rightarrow \infty$, does not scale with the size of the system since

$$
\mathrm{P}_{\Lambda}^{(2)}\left[\Psi_{\Omega}\right]=|\Omega|^{-1}>0
$$

is independent of $L$.

In practice, finite size scaling analysis of $\mathrm{P}_{\Lambda}^{(2)}$ does not distinguish extended from localized states close to the mobility edge. Indeed, in numerical simulations, one calculates energy eigenstates whose wave functions have at most isolated zeroes in contrast to, say, $\left|\Psi_{\Omega}\right\rangle$. Moreover, for energies close to the mobility edge, the localization length is so large that the eigenstates, for all intent and purposes, extend throughout the systems accessible to numerical simulations. Close to the mobility edge, it is more appropriate to ask what is the nature of the extended state in the finite size system: is it a state with strongly fluctuating or uniform amplitude? Generalized inverse participation ratios can address this issue. Generalized inverse participation ratios are defined to be functionals on the space of normalized wave functions $\{\Psi\}$ in a volume of linear size $L$

$$
\mathrm{P}_{L}^{(q)}[\Psi] \equiv \int_{\operatorname{supp} \Psi} \frac{d^{d} x}{L^{d}}|\Psi(x)|^{2 q}, \quad \mathrm{P}_{L}^{(1)}[\Psi] \equiv \int_{\operatorname{supp} \Psi} \frac{d^{d} x}{L^{d}}|\Psi(x)|^{2}=1,
$$

taking values in $] 0,1]$ for positive real values of $q$, and in $[1, \infty[$ for negative real values of $q$. The usefulness of generalized inverse participation ratios comes from the property that $\mathrm{P}_{L}^{(q)}[\Psi]$ is dominated by the largest probability density $\sup _{x}\left\{|\Psi(x)|^{2}\right\}$ when $q$ is very positive. Conversely, when $q$ is very negative, the smallest probability density $\inf _{x}\left\{|\Psi(x)|^{2}\right\}$ dominates in $\mathrm{P}_{L}^{(-|q|)}[\Psi]$. Thus, the $q$-th generalized inverse participation ratio for any extended state with uniform amplitude scales with the linear size of the system as

$$
\mathrm{P}_{\Lambda}^{(q)}\left[\Psi_{\Lambda}\right]=L^{-\tau(q)}, \quad \tau(q)=d(q-1)
$$

This is an example of gap scaling. I Any scaling departing from Eq. (5.14) signals that the state is a simple fractal or multifractal. This classification is based on writing

$$
\tau(q)=D(q)(q-1) .
$$

\footnotetext{
${ }^{4}$ See footnote 1 in the introduction.
} 
When $D(q)$ is independent of $q$, the state is said to be simple fractal, in which case gap scaling still holds. Otherwise, it is said to be multifractal and gap scaling breaks down. For a multifractal wave function, the supports of its maxima and minima scale with the system size with two different exponents $D(-\infty)>D(+\infty)$.

The functional form of $\tau(q)$ is severely constrained by the fact that the wave function $\Psi(x)$ induces a normalized probability measure $|\Psi(x)|^{2}$. By construction $\tau(q)$ is strictly increasing and vanishes when $q=1$ due to the normalization condition. For our purposes, the most important property of the function $\tau(q)$ is that it is concave. Multifractality of the wave function is equivalent to the strict concavity of the function $\tau(q)$. Other analytical properties of $\tau(q)$ can be found in Ref. [10].

In numerical experiments on some two-dimensional non-interacting tight-binding Hamiltonians with static disorder [10], the collection of $q$-th generalized inverse participation ratios $\left\{P_{L}^{(q)}\left[\Psi_{E, V}\right]\right\}_{V}$ is calculated for some collection of normalized energy eigenfunctions labelled by the impurity potential $V$, i.e., $\left(H_{0}+V\right)\left|\Psi_{E, V}\right\rangle=E\left|\Psi_{E, V}\right\rangle$. It is observed that,

$$
P_{L}^{(q)}\left[\Psi_{E, V}\right] \approx L^{-\tau(q)}, \quad \forall V,
$$

for energies close to the critical energy $E_{\text {cr }}$, i.e., when $1 \ll L \ll L_{E}$ where $L_{E}$ is the localization length. We therefore make the hypothesis that at criticality and in the infinite volume limit $\ln P^{(q)}\left[\Psi_{E_{\mathrm{cr}}, V}\right]$ is a selfaveraging random variable:

$$
\lim _{L \rightarrow \infty} \frac{\overline{\ln P_{L}^{(q)}\left[\Psi_{E_{\mathrm{cr}}, V}\right]}}{-\ln L}=\lim _{L \rightarrow \infty} \frac{\ln P_{L}^{(q)}\left[\Psi_{E_{\mathrm{cr}}, V}\right]}{-\ln L}=\tau(q) .
$$

(The overbar denotes averaging over the random potential $V$.) If Eq. (5.17) holds, it is not necessary to perform an averaging over the disorder to calculate $\tau(q)$, as long as the system size $L$ is chosen large enough. The challenge for a field theory describing the universal properties of the multifractal critical wave function $\Psi_{E_{\mathrm{cr}}, V}(x)$ is to obtain the scaling exponents $\{\tau(q) \mid q \in \mathbb{R}\}$.

To illustrate the difficulties involved in calculating from a critical field theory the function $\tau(q)$ defined by Eq. (5.17), we use the definition of Wegner [12] for disorder averaged generalized inverse participation ratios

$$
\mathcal{P}^{(q)}(\omega) \equiv \overline{\overline{\sum_{x, i}\left|\left\langle x \mid \Psi_{i, V}\right\rangle\right|^{2 q} \delta\left(\omega-\omega_{i}\right)}}, \quad q \geq 0,
$$

and apply it to Dirac fermions with random vector potentials. Here, $i$ labels a complete basis of energy eigenstates $\left\{\left|\Psi_{i, V}\right\rangle\right\}_{i}$ for a given realization of the static random vector potential $V$ defined in Eq (4.2). The denominator is the density of states per energy. It insures that $\mathcal{P}^{(2)}(\omega)$ is normalized to one. The right-hand-side of Eq. (5.18) equals $\overline{P_{L_{\omega_{i}}}^{(q)}\left[\Psi_{\omega_{i}, V}\right]}$ if and only if the wave function $\Psi_{\omega_{i}, V}(x)$ has been normalized to one. Although for $q>0 P_{L_{\omega_{i}}}^{(q)}\left[\Psi_{\omega_{i}, V}\right]$ takes values in $] 0,1\left[, \mathcal{P}^{(q)}(\omega)\right.$ takes values in $] 0, \infty[$, since the condition that the wave functions entering Eq. (5.18) are normalized has been relaxed. We now show that for energies asymptotically close to the critical energy for Dirac fermions, namely $\omega=0$, each $\mathcal{P}^{(q)}(\omega)$ scales like $|\omega|^{\varpi(q)}$. We will then discuss the relationship between the set of scaling exponents $\left\{\tau^{*}(q) \equiv z \varpi(q) \mid q \in \mathbb{N}\right\}$ calculated with the critical field theory of the previous sections and the scaling exponents $\{\tau(q) \mid q \in \mathbb{N}\}$ defined by Eq. (5.17) (i.e., derived from the normalized wave functions with vanishing energy of Dirac fermions with random vector potentials).

We first assume that, after averaging over disorder, each term in the numerator is independent of the spatial coordinate $x$ in Eq. (5.18). If so, the summation over the spatial coordinates can be dropped in the numerator, if the denominator is replaced by the averaged local density of states $\rho(\omega)$. The usefulness of Eq. (5.18) then comes from the identity [12]

$$
\mathcal{P}^{(q)}(\omega) \rho(\omega)=\frac{1}{C_{q}} \lim _{\eta \rightarrow 0^{+}}\left[\eta^{q-1} A^{(q)}(\omega, \eta)\right], \quad q \in \mathbb{N},
$$

since we can relate the right-hand-side to a local operator in the critical field theory for Dirac fermions. To see this, recall that according to Eq. (2.4), $A^{(q)}(\omega, \eta)$ is the disorder average over the $q$-th power of the smeared density of 
states per volume and per energy for the complex energy $E=\omega+$ i $\eta$. 5 We now calculate the scaling exponent of $\mathcal{P}^{(q)}(\omega)$ with respect to $\omega$ as we did for the averaged local density of states. We infer from Eqs. (2.4), (3.32), (3.34), (4.48b), and (5.3) the transformation law obeyed by $\eta^{q-1} A^{(q)}(0,0)$ under real space rescaling on the critical line $g_{A} \geq 0, g_{B}=\infty$, namely

$$
\tilde{\eta}^{q-1}\left(\tilde{\varphi}_{+}^{\dagger} \tilde{\varphi}_{-}\right)^{q}(\tilde{x})=a^{-z(q-1)-q+\frac{g_{A}}{\pi} q^{2}+\frac{N-1}{N^{2}}\left(q^{2}+N q\right)} \eta^{q-1}\left(\varphi_{+}^{\dagger} \varphi_{-}\right)^{q}(x), \quad a>0 .
$$

With the help of Eqs. (5.4b), and (5.5), we arrive to the scaling obeyed by $\mathcal{P}^{(q)}(\omega)$ to lowest order in $\omega$

$$
\begin{aligned}
& \mathcal{P}^{(q)}(\omega) \propto|\omega|^{\varpi(q)}, \\
& \varpi(q)=\frac{\tau^{*}(q)}{z}, \quad \tau^{*}(q)=D^{*}(q)(q-1), \quad D^{*}(q)=2-\left(\frac{g_{A}}{\pi}+\frac{N-1}{N^{2}}\right) q .
\end{aligned}
$$

It is remarkable that the same continuous spectrum of exponents

$$
\varpi(q)=\frac{\tau^{*}(q)}{z}, \quad \tau^{*}(q)=D^{*}(q)(q-1), \quad D^{*}(q)=2-\frac{g_{A}}{\pi} q,
$$

along the critical line $g_{A} \geq 0, g_{B}=0$, has been obtained by Ludwig et al. in a very different manner [20]. They first found an eigenstate with vanishing energy for any realization of the Abelian vector gauge-like impurity potential. They then normalized the wave function in a square box of linear dimension $L$, thus obtaining the real and strictly positive wave function $\Psi_{0, V}(x)$. Finally, they calculated how the disorder average $\overline{\left|\Psi_{0, V}(x)\right|^{2 q}}$ scales with the box size $L$ using the replica trick to treat the normalization constant, and assuming that this constant is selfaveraging. However, as we will discuss below, the normalization constant is not selfaveraging. Thus, the calculation in Ref. 20] cannot be viewed as a calculation of $\tau(q)$ defined by Eq. (5.17).

Using the definition for averaged generalized inverse participation ratios Eq. (5.18), we showed in Eq. (5.21b) that they obey a scaling law controlled by local operators in the critical field theory for Dirac fermions with random vector potentials. The scaling exponents $\left\{\tau^{*}(q) \mid q \in \mathbb{N}\right\}$ have been obtained non-perturbatively. By that we mean that $\tau^{*}(q)$ is obtained from the exact scaling dimensions $\Delta_{q}$ of local operators $\left(\varphi_{+}^{\dagger} \varphi_{-}\right)^{q}$ in the critical field theory (see Eq. (5.35)). Both the scaling exponents $\left\{\tau^{*}(q) \mid q \in \mathbb{N}\right\}$ defined by Eq. (5.21b) and the scaling exponents $\{\tau(q) \mid q \in \mathbb{N}\}$ defined by Eq. (5.17) characterize the statistical properties of the collection of critical wave functions $\left\{\Psi_{0, V}(x)\right\}_{V}$. Hence, they should be closely related although they need not be equal. In fact, they cannot be equal since Eq. (5.21b) implies that the scaling exponent $\tau^{*}(q)$ becomes negative for large enough $q$. In contrast, $\tau(q)$ must be positive. There are two reasons for this obvious difference. First, the critical wave functions entering Eq. (5.17) are normalized whereas they are not in Eq. (5.21b). Second, the normalization factor for unnormalized critical wave function is not a selfaveraging quantity for Dirac fermions with random vector potentials:

$$
\lim _{L \rightarrow \infty} \overline{\int d^{2} x\left|\Psi_{0, V}(x)\right|^{2}} \neq \lim _{L \rightarrow \infty} \int d^{2} x\left|\Psi_{0, V}(x)\right|^{2}, \quad \forall V .
$$

For large $q$, rare events, which are defined by $\left|\Psi_{0, V}(x)\right|^{2}$ being much larger than $\overline{\left|\Psi_{0, V}(x)\right|^{2}}$, dominate the average in $\mathcal{P}^{(q)}(\omega)$. Consequently, $\mathcal{P}^{(q)}(\omega)$ can diverge in the limit $\omega \rightarrow 0$.

It turns out that the issue of selfaveraging is far more important than the issue of normalization. Indeed, even if we had required in Eq. (5.18) that the wave functions be normalized to one, the corresponding scaling exponents $\left\{\tau^{* *}(q) \mid q \in \mathbb{N}\right\}$ could only equal the scaling exponents $\{\tau(q) \mid q \in \mathbb{N}\}$ if generalized inverse participation ratios were selfaveraging. However, the parabolic shape of $\tau^{*}(q)$ strongly suggests that this is not the case, since the parabolic shape is characteristic of a log-normal distributed random variable 20,10]. Conversely, if the critical wave function is multifractal (i.e., $\tau(q)$ is strictly concave), then all averaged generalized inverse participation ratios are necessarily non-selfaveraging. By enforcing the constraint of normalization of the wave functions in Eq. (5.18), the new set of scaling exponents $\tau^{* *}(q)$ thus obtained must be positive, but they need not equal $\tau(q)$. [6 We must then conclude that for Dirac fermions with random vector potentials

\footnotetext{
${ }^{5}$ The derivation of the combinatoric factor $C_{q}$ can be found in Ref. 12. Multiplication by $\eta^{q-1}$ and taking the limit $\eta \rightarrow 0^{+}$ projects out of $A^{(q)}(\omega, \eta)$ contributions not present in $\mathcal{P}^{(q)}(\omega)$.

${ }^{6}$ The Legendre transform $f^{* *}(\alpha) \equiv \alpha q-\tau^{* *}(q)$, where $\alpha=\frac{d \tau^{* *}(q)}{q}$, has been shown to take negative values in a model of random resistors [55]. On the other hand, $f(\alpha) \equiv \alpha q-\tau(q)$, where $\alpha=\frac{d \tau(q)}{q}$, is always positive [10].
} 


$$
\left\{\tau^{*}(q) \mid q \in \mathbb{N}\right\} \neq\left\{\tau^{* *}(q) \mid q \in \mathbb{N}\right\} \neq\{\tau(q) \mid q \in \mathbb{N}\}
$$

although we believe that the strict concavity of any one of $\tau^{*}(q), \tau^{* *}(q), \tau(q)$ imply that of the two others.

We close this subsection by reexamining an attempt made in Ref. [11] to relate multifractal scaling exponents to the scaling dimensions of powers of local operator in critical field theories. Let $\Psi_{0, V}(x)$ be a critical eigenfunction of the Dirac Hamiltonian with random vector potential $V$ in a square box of linear dimension $L$. Define the so-called local random event

$$
O_{0, V}(x)=\left|\Psi_{0, V}(x)\right|^{2} .
$$

We want to construct a normalized probability measure out of local random events. We therefore define the so-called local probability 11]

$$
\mu_{0, V}(x)=\frac{O_{0, V}(x)}{\int_{L^{2}} \frac{d^{2} x}{L^{2}} O_{0, V}(x)}
$$

We can always write

$$
\int_{L^{2}} \frac{d^{2} x}{L^{2}} \mu_{0, V}^{q}(x)=\left(\frac{\varepsilon}{L}\right)^{\tau(q)} F_{0, V}\left(q, \frac{\varepsilon}{L}\right), \quad q \in \mathbb{R},
$$

where $\varepsilon$ is some microscopic length scale. We made in Eq. (5.17) the assumption that $\ln F_{0, V}\left(q, \frac{\varepsilon}{L}\right)$ depends only weakly on $L$, i.e.,

$$
\lim _{L \rightarrow \infty} \frac{\ln F_{0, V}\left(q, \frac{\varepsilon}{L}\right)}{\ln L}=\lim _{L \rightarrow \infty} \frac{\overline{\ln F_{0, V}\left(q, \frac{\varepsilon}{L}\right)}}{\ln L}=0, \quad q \in \mathbb{R} .
$$

Note that Eq. (5.28) does not rule out the possibility that for any $q \in \mathbb{R}$ averaging over randomness (denoted by an overbar) yields

$$
\lim _{L \rightarrow \infty} \overline{F_{0, V}\left(q, \frac{\varepsilon}{L}\right)}=F_{0}(q) \lim _{L \rightarrow \infty}\left(\frac{\varepsilon}{L}\right)^{\delta \tau(q)}, \quad \delta \tau(q) \in \mathbb{R} .
$$

Let us make instead of Eq. (5.28) the much stronger assumption that the amplitude $F_{0, V}\left(q, \frac{\varepsilon}{L}\right)$ does not depend on the randomness $V$ and on the system size in the thermodynamic limit:

$$
\lim _{L \rightarrow \infty} F_{0, V}\left(q, \frac{\varepsilon}{L}\right)=F_{0}(q), \quad q \in \mathbb{R},
$$

i.e., $\mu_{0, V}^{q}(x)$ is selfaveraging. This is the assumption made in Eqs. (1-3) of Ref. 11. Unfortunately, this assumption does not apply to our model of Dirac fermions with random vector potentials for which Eq. (5.29) with $\delta \tau(q)=$ $\tau^{*}(q)-\tau(q)$ holds. It is thus incorrect to use Eq. (3) of Ref. [11], namely to equate (see Eq. (5.35) $\Delta_{q}-2-q\left(\Delta_{1}-2\right.$ ) with $\tau(q)$, as was done in Ref. [20]. On the other hand, Eq. (5.30) should yield a good approximation to $\tau(q)$ in the vicinity of $q=0$, since $\mu_{0, V}^{q}(x)=\exp \left[q \ln \mu_{0, V}(x)\right] \approx 1+q \ln \mu_{0, V}(x)$ is very close to being selfaveraging for small $q$.

\section{Scaling of averaged spatial correlations of generalized inverse participation ratios}

In the previous subsection, we have constructed a spectrum of exponents $\left\{\tau^{*}(q) \mid q \in \mathbb{N}\right\}$ which characterizes the scaling of averaged generalized inverse participation ratios. There exists a linear relation between the $\tau^{*}(q)$ 's and the scaling dimensions $\left\{\Delta_{q} \mid q \in \mathbb{N}\right\}$, Eq. (5.35), of some local operators in the critical field theory. In this subsection, we study the interplay between the operator product expansion and the strict concavity of $\Delta_{q}$ by constructing averaged spatial correlations of generalized inverse participation ratios.

Following Ref. [13], averaged spatial correlations of generalized inverse participation ratios are defined by

$$
\mathcal{Q}^{\left(q_{1}, q_{2}\right)}(x, y, \omega) \equiv \frac{\overline{\sum_{i}\left|\left\langle x \mid \Psi_{i}\right\rangle\right|^{2 q_{1}}\left|\left\langle y \mid \Psi_{i}\right\rangle\right|^{2 q_{2}} \delta\left(\omega-\omega_{i}\right)}}{\overline{\sum_{i}\left|\left\langle x \mid \Psi_{i}\right\rangle\right|^{2} \delta\left(\omega-\omega_{i}\right)}}, \quad q_{1}, q_{2}>0
$$

The $q$-th generalized inverse participation ratio is recovered by choosing $q_{1}=q, q_{2}=0$. Similarly to Eq. (5.32), we use the identity [13] 


$$
\mathcal{Q}^{\left(q_{1}, q_{2}\right)}(x, y, \omega) \rho(\omega)=\frac{1}{C_{q_{1}+q_{2}}} \lim _{\eta \rightarrow 0^{+}}\left[\eta^{q_{1}+q_{2}-1} A^{\left(q_{1}, q_{2}\right)}(x, y, \omega, \eta)\right] .
$$

Here, $A^{\left(q_{1}, q_{2}\right)}(x, y, \omega, \eta)$, which is defined by

$$
A^{\left(q_{1}, q_{2}\right)}(x, y, \omega, \eta) \equiv \overline{\left\{\frac{\operatorname{Im}}{\pi} \operatorname{tr}[G(x, x, \omega-\mathrm{i} \eta ; V)]\right\}^{q_{1}}\left\{\frac{\operatorname{Im}}{\pi} \operatorname{tr}[G(y, y, \omega-\mathrm{i} \eta ; V)]\right\}^{q_{2}}}
$$

should be compared to Eq. (2.4). We calculate the scaling of $A^{\left(q_{1}, q_{2}\right)}(x, y, \omega, \eta)$ with respect to large separation $|x-y|$ and with respect to small $\omega \neq 0$. In other words, we work in the regime

$$
\varepsilon \ll|x-y| \ll L_{\omega}, \quad L_{\omega} \propto|\omega|^{-z} .
$$

Here, $\varepsilon$ is the ultraviolet cutoff, say, the lattice spacing. Of course, $\eta>0$ can be taken as small as needed. Scaling is calculated as follows [13]. The first step is to perform a rescaling which brings the separation down to the lattice spacing $\varepsilon$. The second step makes use of the operator product expansion. The final step is to perform a rescaling to energy scales $\bar{\omega}$ and $\bar{\eta}$ which are arbitrarily chosen. Under each rescaling, we use the scaling dimensions of the appropriate operators. In particular, to lowest order in $\omega$, we use the scaling dimensions at criticality (see Eqs. $(3.30 \mathrm{~b}, 4.48 \mathrm{~b})$ )

$$
\Delta_{q}=q-\frac{g_{A}}{\pi} q^{2}-\frac{N-1}{N^{2}}\left(q^{2}+N q\right)
$$

for the operators $\left(\varphi_{+}^{\dagger} \varphi_{-}\right)^{q_{1}}(x),\left(\varphi_{+}^{\dagger} \varphi_{-}\right)^{q_{2}}(y)$, and $\left(\varphi_{+}^{\dagger} \varphi_{-}\right)^{q_{1}+q_{2}}(\varepsilon)$, respectively.

Under rescaling, $A^{\left(q_{1}, q_{2}\right)}(x, y, \omega, \eta)$ transforms like

$$
\left\langle\left(\varphi_{+}^{\dagger} \varphi_{-}\right)^{q_{1}}(x)\left(\varphi_{+}^{\dagger} \varphi_{-}\right)^{q_{2}}(y)\right\rangle_{S_{\text {eff }}^{(1,0)}(\omega-\mathrm{i} \eta)},
$$

according to section $\mathrm{III}$. Hence, under the rescaling

$$
\tilde{x}=a x, \quad \tilde{y}=a y, \quad \tilde{\omega}=a^{-z} \omega, \quad \tilde{\eta}=a^{-z} \eta, \quad a \equiv \frac{\varepsilon}{|x-y|} \ll 1,
$$

we find that

$$
\begin{aligned}
A^{\left(q_{1}, q_{2}\right)}(x, y, \omega, \eta) & \sim\left(\frac{\varepsilon}{|x-y|}\right)^{\Delta_{q_{1}}+\Delta_{q_{2}}}\left\langle\left(\tilde{\varphi}_{+}^{\dagger} \tilde{\varphi}_{-}\right)^{q_{1}}(\tilde{x})\left(\tilde{\varphi}_{+}^{\dagger} \tilde{\varphi}_{-}\right)^{q_{2}}(\tilde{y})\right\rangle_{S_{\text {eff }}^{(1,0)}(\tilde{\omega}-\mathrm{i} \tilde{\eta})} \\
& \sim\left(\frac{\varepsilon}{|x-y|}\right)^{\Delta_{q_{1}}+\Delta_{q_{2}}}\left\langle\left(\tilde{\varphi}_{+}^{\dagger} \tilde{\varphi}_{-}\right)^{q_{1}+q_{2}}(\tilde{y})\right\rangle_{S_{\text {eff }}^{(1,0)}(\tilde{\omega}-\mathrm{i} \tilde{\eta})} \cdot
\end{aligned}
$$

On the second line of Eq. (5.38), we have performed an operator product expansion from which only the most relevant operator of the expansion is kept. Notice that the identity operator is not the most relevant operator in the operator product expansion. It would have been, had we considered, say, the pure Gaussian field theory with the hierarchy of operators $\exp (q \mathrm{i} \phi)$ and the positive and convex spectrum of exponents $\left\{q^{2} \mid q \in \mathbb{N}\right\}$. The two factors on the right-hand-side of Eq. (5.38) both depends on the separation $|x-y|$. However, the rescaling to the arbitrarily chosen energy $\bar{\omega}<\omega$

$$
\bar{y}=b \tilde{y}, \quad \bar{\omega}=b^{-z} \tilde{\omega}, \quad \bar{\eta}=b^{-z} \tilde{\eta}, \quad b=\frac{1}{a}\left|\frac{\bar{\omega}}{\omega}\right|^{-\frac{1}{z}}
$$

yields

$$
\begin{aligned}
A^{\left(q_{1}, q_{2}\right)}(x, y, \omega, \eta) & \sim\left(\frac{\varepsilon}{|x-y|}\right)^{\Delta_{q_{1}}+\Delta_{q_{2}}-\Delta_{q_{1}+q_{2}}}\left|\frac{\omega}{\bar{\omega}}\right|^{\frac{1}{z} \Delta_{q_{1}+q_{2}}}\left\langle\left(\bar{\varphi}_{+}^{\dagger} \bar{\varphi}-\right)^{q_{1}+q_{2}}(\bar{y})\right\rangle_{S_{\mathrm{eff}}^{(1,0)}(\bar{\omega}-\mathrm{i} \bar{\eta})} \\
& \equiv\left(\frac{\varepsilon}{|x-y|}\right)^{\Delta_{q_{1}}+\Delta_{q_{2}}-\Delta_{q_{1}+q_{2}}}\left|\frac{\omega}{\bar{\omega}}\right|^{\frac{1}{z} \Delta_{q_{1}+q_{2}}} F^{\left(q_{1}, q_{2}\right)}(\bar{\omega}, \bar{\eta}) .
\end{aligned}
$$


The scaling function $F^{\left(q_{1}, q_{2}\right)}(\bar{\omega}, \bar{\eta})$ is defined by Eq. (5.40). It is given by the expectation value at energy $\bar{\omega}-\mathrm{i} \bar{\eta}$ of the most relevant operator in the operator product expansion in Eq. (5.38). By assumption, it does not depend on $\bar{y}$. For given $q_{1,2}$, it therefore only depends on $\bar{\omega}$ and $\bar{\eta}$. Calculating $F^{\left(q_{1}, q_{2}\right)}(\bar{\omega}, \bar{\eta})$ is beyond the scope of our approach. However, some of its limiting behaviours can be inferred from the fact that $F^{\left(q_{1}, q_{2}\right)}(\bar{\omega}, \bar{\eta})$ enters an averaged generalized inverse participation ratio.

We consider the limit $\omega>0, \eta \rightarrow 0$. Because we are working in the thermodynamic limit at finite real energy $\omega$, the energy eigenstates must be localized. Hence, $\mathcal{Q}^{\left(q_{1}, q_{2}\right)}(x, y, \omega)$ must be finite so that from Eq. (5.32)

$$
\lim _{\eta \rightarrow 0} \eta^{q_{1}+q_{2}-1} F^{\left(q_{1}, q_{2}\right)}(\bar{\omega}, \bar{\eta}) \sim\left(\frac{\eta}{\bar{\eta}}\right)^{q_{1}+q_{2}-1} G^{\left(q_{1}, q_{2}\right)}(\bar{\omega}) .
$$

This gives us the scaling

$$
\mathcal{Q}^{\left(q_{1}, q_{2}\right)}(x, y, \omega) \rho(\omega) \sim\left(\frac{\varepsilon}{|x-y|}\right)^{\Delta_{q_{1}}+\Delta_{q_{2}}-\Delta_{q_{1}+q_{2}}}\left|\frac{\omega}{\bar{\omega}}\right|^{\frac{1}{z}\left[\Delta_{q_{1}+q_{2}}+z\left(q_{1}+q_{2}-1\right)\right]} G^{\left(q_{1}, q_{2}\right)}(\bar{\omega}) .
$$

This scaling has two important features. First, the exponent characterizing the scaling with separation is always positive since

$$
\Delta_{q_{1}}+\Delta_{q_{2}}-\Delta_{q_{1}+q_{2}}=2\left(\frac{g_{A}}{\pi}+\frac{N-1}{N^{2}}\right) q_{1} q_{2}, \quad q_{1}, q_{2}>0 .
$$

Secondly, the exponent characterizing the scaling with respect to $\omega$ is that of the $\left(q_{1}+q_{2}\right)$-th generalized inverse participation ratio:

$$
\frac{1}{z}\left[\Delta_{q_{1}+q_{2}}+z\left(q_{1}+q_{2}-1\right)\right]=\varpi\left(q_{1}+q_{2}\right)+\frac{2-z}{z} .
$$

Consequently, we recover the scaling for generalized inverse participation ratios when either $q_{1}$ or $q_{2}$ vanishes as it should be.

Eq. (5.42) tells us that the property of strict concavity $\Delta_{q_{1}}+\Delta_{q_{2}}-\Delta_{q_{1}+q_{2}}>0, q_{1}, q_{2}>0$, insures that spatial correlations decrease with separation. Negative dimensional scaling exponents also control the scaling with respect to energy, or, equivalently, with respect to the effective system size. This latter scaling implies that the normalization of the local observable can blow up with the system size in the thermodynamic limit. In fact, Eq. (5.42) reproduces the scaling for spatial correlations of so-called non-normalizable box-observables [39,11, 10]. Also note that the identity operator never enters as the most relevant operator in the operator product expansion between pairs of local operators with scaling dimensions $\Delta_{q_{1}}$ and $\Delta_{q_{2}}$, and this, in turn, is guaranteed by $\Delta_{q}$ becoming negative for sufficiently large $q$. In contrast, it is much more difficult to find a critical point characterized by an infinite set of positive scaling exponents $\left\{x_{q}>0 \mid q \in \mathbb{N}\right\}$, which are not linearly related, and such that $x_{q_{1}+q_{2}}>0$ is generated from $x_{q_{1}}$ and $x_{q_{2}}$ as the most relevant operator in the operator product expansion of their associated operators [11.

In summary, several scaling properties of the multifractal measure induced by the critical wave function of Dirac fermions with random vector potentials are shared by the probability distribution corresponding to averaged generalized inverse participation ratios. In the literature it is assumed that the strict concavity of $\tau^{*}(q)$ for small $q$ implies that of $\tau(q)$. Although this assumption seems reasonable, it would be highly desirable to verify it by reconstructing $\{\tau(q) \mid q \in \mathbb{N}\}$ from $\left\{\tau^{*}(q) \mid q \in \mathbb{N}\right\}$. Moreover, by doing so it could be established whether or not the existence of negative dimensional local operators implies multifractality of the critical wave function.

\section{CURRENT ALGEBRA FOR GRADED SYMMETRIES: $U(1 / 1)$ CURRENT ALGEBRA}

In the previous sections, we have studied some disordered critical points through their fixed point Lagrangians. In this section, we take a very different approach. We want to describe new disordered critical points using conformal and internal symmetries and the associated current algebra. We will see how the current algebra approach allows us to access new fixed points which cannot be reached perturbatively from some known fixed point (contrast this approach to that in Refs. [18, 19]).

Current algebra (and the associated bosonization technique) is a powerful method for studying strongly correlated electrons in one spatial dimension $\mid 56$. Current algebra is also very useful for studying critical points in two dimensions which are characterized by certain continuous symmetries. Effective theories for non-interacting disordered systems 
have a graded symmetry (or supersymmetry) [28]. For example, we have seen in section II that disorder average over products of Green functions can equally well be represented by path integrals over fermionic or bosonic coherent states. In this section, we study the current algebra of graded symmetry in two dimensions. We classify all critical points for two-dimensional systems with unbroken $U(1 / 1)$ graded symmetry. We recover the critical points corresponding to $U(1)$ vector gauge-like disorder studied in the previous sections. We also find new critical points. The operator content for these critical points is constructed. In particular, we show that most critical points are characterized by infinitely many local operators with negative dimensions.

In the current algebra approach, one first constructs the commutator and anticommutator algebra for the conserved currents. At a critical point, conformal symmetry allows one to calculate all correlations between the currents from the commutator and anticommutator algebra. In this way, the theory is completely specified.

\section{A. Current algebra description of a free model}

We begin with the simplest model with graded $U(1 / 1)$ symmetry. It is given by the partition function

$$
\begin{aligned}
& \mathcal{Z}_{0}=\int \mathcal{D}\left[\psi_{ \pm}^{\dagger}, \psi_{ \pm}\right] \int \mathcal{D}\left[\varphi_{ \pm}^{\dagger}, \varphi_{ \pm}\right] e^{\mathrm{i} \int_{-\infty}^{+\infty} d x d \tau \mathcal{L}_{0}} \\
& \mathcal{L}_{0}=\frac{\mathrm{i}}{\pi}\left(\psi_{+}^{\dagger} \partial_{\bar{z}} \psi_{+}+\varphi_{+}^{\dagger} \partial_{\bar{z}} \varphi_{+}+\psi_{-}^{\dagger} \partial_{z} \psi_{-}+\varphi_{-}^{\dagger} \partial_{z} \varphi_{-}\right) .
\end{aligned}
$$

Here, $(x, \tau)$ denotes the coordinates of two-dimensional Euclidean space, and the fields are the chiral components of Dirac spinors introduced in Eq. (3.15). For convenience, we have rescaled the spinors by the factor $\sqrt{2 \pi}$. The only non-vanishing two-point Green functions are

$$
\begin{aligned}
\left\langle\psi_{+}^{\dagger}(z) \psi_{+}(0)\right\rangle & =\left\langle\psi_{+}(z) \psi_{+}^{\dagger}(0)\right\rangle=\frac{1}{z}, \\
-\left\langle\varphi_{+}^{\dagger}(z) \varphi_{+}(0)\right\rangle & =\left\langle\varphi_{+}(z) \varphi_{+}^{\dagger}(0)\right\rangle=\frac{1}{z}, \\
\left\langle\psi_{-}^{\dagger}(\bar{z}) \psi_{-}(0)\right\rangle & =\left\langle\psi_{-}(\bar{z}) \psi_{-}^{\dagger}(0)\right\rangle=\frac{1}{\bar{z}} \\
-\left\langle\varphi_{-}^{\dagger}(\bar{z}) \varphi_{-}(0)\right\rangle & =\left\langle\varphi_{-}(\bar{z}) \varphi_{-}^{\dagger}(0)\right\rangle=\frac{1}{\bar{z}}
\end{aligned}
$$

where $z=x+\mathrm{i} \tau$ and $\bar{z}=x-\mathrm{i} \tau$ are the independent holomorphic and antiholomorphic coordinates, respectively.

\section{Symmetries of $\mathcal{L}_{0}$}

The action in Eq. (6.1) is left invariant by the global chiral transformation

$$
\psi_{+}^{\dagger}=e^{-\mathrm{i} \theta_{+}^{\psi}} \psi_{+}^{\prime \dagger}, \quad \psi_{+}=e^{+\mathrm{i} \theta_{+}^{\psi}} \psi_{+}^{\prime}
$$

where $\theta_{+}^{\psi}$ is any arbitrary real number. The associated current

$$
J_{z}^{\psi}(z)=\left(\psi_{+}^{\dagger} \psi_{+}\right)(z)
$$

is conserved, i.e., its conservation is equivalent to it being a holomorphic function

$$
\partial_{\bar{z}} J_{z}^{\psi}(z)=0
$$

Similarly, there are three additional independent global $U(1)$ chiral symmetries with the conserved currents

$$
J_{\bar{z}}^{\psi}(\bar{z})=\left(\psi_{-}^{\dagger} \psi_{-}\right)(\bar{z}), \quad J_{z}^{\varphi}(z)=\left(\varphi_{+}^{\dagger} \varphi_{+}\right)(z), \quad J_{\bar{z}}^{\varphi}(\bar{z})=\left(\varphi_{-}^{\dagger} \varphi_{-}\right)(\bar{z}),
$$

respectively. The action in Eq. (6.1) is also left invariant by the four independent infinitesimal transformations

$$
\delta_{\eta} \psi_{ \pm}^{\dagger} \equiv\left[\bar{\theta}_{ \pm} \eta_{ \pm}, \psi_{ \pm}^{\dagger}\right]=+\varphi_{ \pm}^{\dagger} \bar{\theta}_{ \pm}, \quad \delta_{\eta} \varphi_{ \pm} \equiv\left[\bar{\theta}_{ \pm} \eta_{ \pm}, \varphi_{ \pm}\right]=-\bar{\theta}_{ \pm} \psi_{ \pm}
$$


and

$$
\delta_{\bar{\eta}} \varphi_{ \pm}^{\dagger} \equiv\left[\theta_{ \pm} \bar{\eta}_{ \pm}, \varphi_{ \pm}^{\dagger}\right]=-\psi_{ \pm}^{\dagger} \theta_{ \pm}, \quad \delta_{\bar{\eta}} \psi_{ \pm} \equiv\left[\theta_{ \pm} \bar{\eta}_{ \pm}, \psi_{ \pm}\right]=+\theta_{ \pm} \varphi_{ \pm},
$$

respectively. The four infinitesimal Grassmann numbers $\bar{\theta}_{+}, \bar{\theta}_{-}, \theta_{+}$, and $\theta_{-}$, are independent. The associated conserved fermionic currents are

$$
\eta_{+}=\varphi_{+}^{\dagger} \psi_{+}, \quad \eta_{-}=\varphi_{-}^{\dagger} \psi_{-}, \quad \bar{\eta}_{+}=\psi_{+}^{\dagger} \varphi_{+}, \quad \bar{\eta}_{+}=\psi_{-}^{\dagger} \varphi_{-} .
$$

Taken together, these eight conserved currents, of which four are bosonic and four are fermionic, generate a graded $U(1 / 1) \times U(1 / 1)$ symmetry. The bosonic generators of the diagonal subgroup $U(1 / 1)$ are the currents $J_{\mu}$ and $j_{\mu}$ with

$$
\frac{1}{2}\left(\begin{array}{c}
J_{x}-\mathrm{i} J_{\tau} \\
J_{x}+\mathrm{i} J_{\tau}
\end{array}\right) \equiv\left(\begin{array}{c}
J_{z} \\
J_{\bar{z}}
\end{array}\right) \equiv\left(\begin{array}{c}
J_{z}^{\psi}+J_{z}^{\varphi} \\
J_{\bar{z}}^{\psi}+J_{\bar{z}}^{\varphi}
\end{array}\right), \quad \frac{1}{2}\left(\begin{array}{c}
j_{x}-\mathrm{i} j_{\tau} \\
j_{x}+\mathrm{i} j_{\tau}
\end{array}\right) \equiv\left(\begin{array}{c}
j_{z} \\
j_{\bar{z}}
\end{array}\right) \equiv\left(\begin{array}{c}
J_{z}^{\psi}-J_{z}^{\varphi} \\
J_{\bar{z}}^{\psi}-J_{\bar{z}}^{\varphi}
\end{array}\right) .
$$

The fermionic generators of the diagonal subgroup $U(1 / 1)$ are the currents $\eta_{\mu}$ and $\bar{\eta}_{\mu}$ with

$$
\frac{1}{2}\left(\begin{array}{c}
\eta_{x}-\mathrm{i} \eta_{\tau} \\
\eta_{x}+\mathrm{i} \eta_{\tau}
\end{array}\right) \equiv\left(\begin{array}{c}
\eta_{z} \\
\eta_{\bar{z}}
\end{array}\right) \equiv\left(\begin{array}{c}
\varphi_{+}^{\dagger} \psi_{+} \\
\varphi_{-}^{\dagger} \psi_{-}
\end{array}\right), \quad \frac{1}{2}\left(\begin{array}{c}
\bar{\eta}_{x}-\mathrm{i} \bar{\eta}_{\tau} \\
\bar{\eta}_{x}+\mathrm{i} \bar{\eta}_{\tau}
\end{array}\right) \equiv\left(\begin{array}{l}
\bar{\eta}_{z} \\
\bar{\eta}_{\bar{z}}
\end{array}\right) \equiv\left(\begin{array}{c}
\psi_{+}^{\dagger} \varphi_{+} \\
\psi_{-}^{\dagger} \varphi_{-}
\end{array}\right) .
$$

Each component of the bosonic and fermionic currents generating $U(1 / 1)$ is separately conserved due to the chiral invariance.

\section{Symmetries of $\mathcal{Z}_{0}$}

The global $U(1 / 1) \times U(1 / 1)$ symmetry of $\mathcal{L}_{0}$ is shared by the measure in the partition function. Hence, $\mathcal{Z}_{0}$ is also left invariant by global $U(1 / 1) \times U(1 / 1)$ transformations. The correlation function $\left\langle A_{1+} \cdots A_{m+} B_{1-} \cdots B_{n-}\right\rangle$, where, say, $\left\{A_{i+}\right\}$ are defined in terms of $\psi_{+}^{\dagger}, \psi_{+}, \varphi_{+}^{\dagger}, \varphi_{+}$, factorizes according to

$$
\left\langle A_{1+} \cdots A_{m+} B_{1-} \cdots B_{n-}\right\rangle=\left\langle A_{1+} \cdots A_{m+}\right\rangle\left\langle B_{1-} \cdots B_{n-}\right\rangle,
$$

due to the decoupling of the + and the - sectors. Moreover, the correlation function corresponding to the + subscript must be holomorphic, whereas the correlation function corresponding to the - subscript must be antiholomorphic. Without loss of generality, we will only consider the holomorphic sector of the theory. The subscript + will be suppressed from now on.

\section{Operator product expansion}

We now study the operator product algebra with the help of the operator product expansion (OPE) [57]. Consider two operators, $A(z)$ and $B(z)$. The OPE of the product $A\left(z_{1}\right) B\left(z_{2}\right)$ is the Laurent series expansion

$$
A\left(z_{1}\right) B\left(z_{2}\right)=\frac{1}{\left(z_{1}-z_{2}\right)^{n}} O_{n}\left(z_{2}\right)+\cdots+\frac{1}{z_{1}-z_{2}} O_{1}\left(z_{2}\right)+\mathcal{O}(1) .
$$

Only the poles in the limit of $z_{1} \rightarrow z_{2}$ are kept in the OPE. In the following, we will always omit the non-diverging term $\mathcal{O}(1)$ in the OPE. For example, the operators $\psi^{\dagger}, \cdots, \varphi$ have the following OPE:

$$
\psi^{\dagger}(z) \psi(0)=\psi(z) \psi^{\dagger}(0)=\varphi(z) \varphi^{\dagger}(0)=-\varphi^{\dagger}(z) \varphi(0)=\frac{1}{z} .
$$

Another example of an OPE is that between the currents $(J, j, \eta, \bar{\eta})$ generating the $U(1 / 1)$ symmetry. However before calculating them, the quantum nature of the currents must be dealt with care. In particular, we must remove any divergences which arise when multiplying operators at the same point. In the context of the currents, such divergences are removed by subtracting from the naive definition of the classical currents in Eqs. (6.8.6.9) the poles in the OPE between the $\psi^{\dagger}, \cdots, \varphi$. For example, the precise definition of $j(z)$ is not given by Eq. (6.8), but by 


$$
\begin{aligned}
j(z) & =\lim _{\epsilon \rightarrow 0}\left[\psi^{\dagger}(z+\epsilon) \psi(z)-\varphi^{\dagger}(z+\epsilon) \varphi(z)-\frac{2}{\epsilon}\right] \\
& \equiv: \psi^{\dagger}(z) \psi(z):-: \varphi^{\dagger}(z) \varphi(z): .
\end{aligned}
$$

The normal ordering : $\cdots$ : extracts the finite contribution from the operator product defining the current $j$, since one can show that the limit $\epsilon \rightarrow 0$ in the correlation function

$$
\left\langle\left[\psi^{\dagger}(z+\epsilon) \psi(z)-\varphi^{\dagger}(z+\epsilon) \varphi(z)\right] \prod_{i} O_{i}\left(z_{i}\right)\right\rangle-\frac{2}{\epsilon}\left\langle\prod_{i} O_{i}\left(z_{i}\right)\right\rangle
$$

is finite and thus defines the correlation function $\left\langle j(z) \prod_{i} O_{i}\left(z_{i}\right)\right\rangle$. With this definition of the currents and with the help of the OPE between $\left\{\psi^{\dagger}, \cdots, \varphi\right\}$, we find the OPE between the currents to be given by

$$
\begin{aligned}
& J(z) J(0)=0, \quad J(z) j(0)=\frac{2}{z^{2}}, \quad J(z) \eta(0)=0, \quad J(z) \bar{\eta}(0)=0, \\
& j(z) J(0)=\frac{2}{z^{2}}, \quad j(z) j(0)=0, \quad j(z) \eta(0)=-\frac{2}{z} \eta(0), \quad j(z) \bar{\eta}(0)=+\frac{2}{z} \bar{\eta}(0), \\
& \eta(z) J(0)=0, \quad \eta(z) j(0)=+\frac{2}{z} \eta(0), \quad \eta(z) \eta(0)=0, \quad \eta(z) \bar{\eta}(0)=-\frac{1}{z^{2}}+\frac{1}{z} J(0), \\
& \bar{\eta}(z) J(0)=0, \quad \bar{\eta}(z) j(0)=-\frac{2}{z} \bar{\eta}(0), \quad \bar{\eta}(z) \eta(0)=+\frac{1}{z^{2}}+\frac{1}{z} J(0), \quad \bar{\eta}(z) \bar{\eta}(0)=0 .
\end{aligned}
$$

An alternative definition of the OPE for the currents can be found in appendix A.

The operator algebra specified by the OPE of Eq. (6.15) is very powerful. Indeed, from the OPE alone, i.e., without any reference to $\mathcal{Z}_{0}$, we can calculate all the correlation functions between the currents $(J, j, \eta, \bar{\eta})$. Here, we assume that expectation values of all currents vanish

$$
\langle J(z)\rangle=\langle j(z)\rangle=\langle\eta(z)\rangle=\langle\bar{\eta}(z)\rangle=0 .
$$

In this sense, the OPE of Eq. (6.15) completely specifies and defines our model. To illustrate the power of the OPE, let us consider the correlation function $\left\langle j\left(z_{1}\right) \bar{\eta}\left(z_{2}\right) \eta\left(z_{3}\right)\right\rangle$. We know that this three-point function is a holomorphic function of $z_{i}$ 's. We may view it as a function of $z_{1}$. If so, this function must have poles at $z_{2}$ and $z_{3}$ and must vanish asymptotically as $z_{1} \rightarrow \infty$. The poles are then completely fixed by the OPE: the three-point function has a first order pole at $z_{2}$ with residue $2\left\langle\bar{\eta}\left(z_{2}\right) \eta\left(z_{3}\right)\right\rangle$, and a first order pole at $z_{3}$ with residue $-2\left\langle\bar{\eta}\left(z_{2}\right) \eta\left(z_{3}\right)\right\rangle$. This completely determines

$$
\left\langle j\left(z_{1}\right) \bar{\eta}\left(z_{2}\right) \eta\left(z_{3}\right)\right\rangle=\frac{2}{z_{1}-z_{2}}\left\langle\bar{\eta}\left(z_{2}\right) \eta\left(z_{3}\right)\right\rangle-\frac{2}{z_{1}-z_{3}}\left\langle\bar{\eta}\left(z_{2}\right) \eta\left(z_{3}\right)\right\rangle .
$$

With the help of $\left\langle\bar{\eta}\left(z_{2}\right) \eta\left(z_{3}\right)\right\rangle=\frac{1}{\left(z_{2}-z_{3}\right)^{2}}$, we find that the three-point function is given by

$$
\left\langle j\left(z_{1}\right) \bar{\eta}\left(z_{2}\right) \eta\left(z_{3}\right)\right\rangle=\frac{2}{\left(z_{1}-z_{2}\right)\left(z_{1}-z_{3}\right)\left(z_{2}-z_{3}\right)} .
$$

It is important to realize that not every possible OPE gives rise to a consistent theory. For example, to calculate the three-point function in Eq. (6.18), we expressed it in terms of two-point functions whose coefficients are determined by the OPE between the currents. But by so doing, we chose a specific decomposition path corresponding to viewing the three-point function as a function of $z_{1}$. One needs to show that different decomposition paths always lead to the same correlation function. In the above example we may instead first view $\left\langle j\left(z_{1}\right) \bar{\eta}\left(z_{2}\right) \eta\left(z_{3}\right)\right\rangle$ as a function of $z_{2}$, which has a first order pole at $z_{1}$ with residue $-2\left\langle\bar{\eta}\left(z_{1}\right) \eta\left(z_{3}\right)\right\rangle$, a second order pole at $z_{3}$ with residue $\left\langle j\left(z_{1}\right)\right\rangle$, and a first order pole at $z_{3}$ with residue $\left\langle j\left(z_{1}\right) J\left(z_{3}\right)\right\rangle$. Thus,

$$
\left\langle j\left(z_{1}\right) \bar{\eta}\left(z_{2}\right) \eta\left(z_{3}\right)\right\rangle=-\frac{2}{z_{2}-z_{1}}\left\langle\bar{\eta}\left(z_{1}\right) \eta\left(z_{3}\right)\right\rangle+\frac{1}{z_{2}-z_{3}}\left\langle j\left(z_{1}\right) J\left(z_{3}\right)\right\rangle .
$$

Comparing Eq. (6.17) with Eq. (6.19), we see that the three-point function can be decomposed into different Laurent series $(\mathrm{OPE})$. Thus, the $\mathrm{OPE}$ between the currents need to satisfy certain self-consistent (associativity) conditions insuring that all current correlation functions are uniquely defined. In our example, this is the case. The self-consistency of the OPE in Eq. (6.15) follows from it being derived from the partition function $\mathcal{Z}_{0}$. 


\section{B. General $U(1 / 1)$ current algebra for interacting models}

We are now ready to describe the philosophy behind the current algebra approach. We know that the model specified by $\mathcal{Z}_{0}$ in Eq. (6.1) describes a critical point characterized by algebraic decaying correlation functions. Moreover, the

representation of $\mathcal{Z}_{0}$ in terms of the free fields $\psi_{+}^{\dagger}, \cdots, \varphi_{-}$allows us to easily calculate all properties of the critical point. On the other hand, for a generic interacting theory such as

$$
\begin{aligned}
& \mathcal{Z}=\int D\left[\psi_{ \pm}^{\dagger}, \psi_{ \pm}\right] \int D\left[\varphi_{ \pm}^{\dagger}, \varphi_{ \pm}\right] e^{\mathrm{i} \int_{-\infty}^{+\infty} d x d \tau\left(\mathcal{L}_{0}+\mathcal{L}_{\mathrm{int}}\right)}, \\
& \mathcal{L}_{\mathrm{int}}=\mathrm{i} \frac{g_{A}}{\pi^{2}}\left(\psi_{+}^{\dagger} \psi_{+}+\varphi_{+}^{\dagger} \varphi_{+}\right)\left(\psi_{-}^{\dagger} \psi_{-}+\varphi_{-}^{\dagger} \varphi_{-}\right)+\mathrm{i} \frac{g_{M}}{4 \pi^{2}}\left(\psi_{+}^{\dagger} \psi_{-}-\varphi_{-}^{\dagger} \varphi_{+}\right)^{2}+\cdots,
\end{aligned}
$$

the interactions drive the system to a new fixed point. The interaction with coupling constant $g_{A}>0$ can be thought of as being induced when integrating over Abelian vector gauge-like disorder in the effective action $S_{\mathrm{cr}}^{(1,0)}$ of Eq. (3.25). Similarly, the interaction with coupling constant $g_{M}>0$ corresponds to integrating over a randomly distributed mass for Dirac fermions. However, from the point of view of the current algebra, $g_{A, M}$ can be negative as we will see later on. Finally, "..." represents other interaction terms. We would like to know what are the critical properties of the new fixed point. The difficulty is that the fields $\psi_{+}^{\dagger}, \cdots, \varphi_{-}$do not provide a convenient description of the new fixed point, making it extremely difficult to calculate its critical properties in terms of $\psi_{+}^{\dagger}, \cdots, \varphi_{-}$. However, it is sometimes possible to take advantage of the fact that the interaction $\mathcal{L}_{\text {int }}$ has a $U(1 / 1)$ graded symmetry. At a critical point of the interacting model, the $U(1 / 1)$ symmetry is promoted to the $U(1 / 1) \times U(1 / 1)$ symmetry in the low energy sector, due to the decoupling of the holomorphic and antiholomorphic sectors at low energy. In this case, many critical properties of the new fixed point can then be derived solely from the knowledge of $U(1 / 1) \times U(1 / 1)$ current algebra. The current algebra approach to a critical point in two dimensions is based on two assumptions: $i$ ) the critical point has a conformal symmetry (which is responsible for promoting the $U(1 / 1)$ to $U(1 / 1) \times U(1 / 1$ ) symmetry), $i i)$ the conserved currents have algebraic decaying correlations, i.e., they belong to gapless sectors.

To construct the critical points with $U(1 / 1) \times U(1 / 1)$ symmetry, we denote the infinitesimal generators of the $U(1 / 1)$ graded symmetry in the holomorphic sector by $\left(I_{1}, I_{2}, I_{3}, I_{4}\right)(z)=(J, j, \eta, \bar{\eta})(z)$. Without loss of generality, we only work in the holomorphic sector. Since conserved currents cannot develop anomalous dimensions, the conformal weights of $I_{a}, a=1, \cdots, 4$ are $(1,0)$. Consequently, the leading pole in the OPE among the $\left\{I_{a}\right\}$ must be of second order, and the most general OPE between the holomorphic components of the generators of the $U(1 / 1)$ graded algebra takes the form

$$
I_{a}(z) I_{b}(0)=\frac{k_{a b}}{z^{2}}+\frac{f_{a b c}}{z} I_{c}+\mathcal{O}(1), \quad a, b=1, \cdots, 4 .
$$

The coefficients $k_{a b}$ of the second order pole contain information about the dynamics of the critical point. We will see below that they are severely restricted by the condition that $k_{a b}$ be an invariant second rank tensor with respect to the $U(1 / 1)$ symmetry. The coefficients $f_{a b c}$ are the structure constants of the graded Lie algebra $U(1 / 1)$.

To see this last point, we introduce the conserved charges

$$
Q_{a}(\tau)=\frac{1}{2 \pi \mathrm{i}} \int_{-\infty}^{+\infty} d x I_{a}(x, \tau), \quad a=1, \cdots, 4,
$$

which generate finite $U(1 / 1)$ transformations. Recalling that correlation functions defined by a path integral corresponds to $\tau$-ordered correlation functions in the operator formalism [58], we find $(a, b=1, \cdots, 4)$

$$
\left\langle\left[Q_{a}(0), Q_{b}(0)\right]_{ \pm} \prod_{i} O\left(x_{i}, \tau_{i}\right)\right\rangle=\left(\frac{1}{2 \pi \mathrm{i}}\right)^{2}\left\langle\int d z \oint_{C} d z^{\prime} I_{a}\left(z^{\prime}\right) I_{b}(z) \prod_{i} O\left(z_{i}\right)\right\rangle
$$

where the integration contour $C$ is a small circle centered at $z$. We have used the fact that $I_{a}$ is a holomorphic function of $z$ in the last line of Eq. (6.23). Care has to be taken in Eq. (6.23) to use the anticommutator $[\cdot, \cdot]_{+}$whenever $Q_{a}$ and $Q_{b}$ are both fermionic, whereas for all other cases the commutator $[\cdot, \cdot]_{-}$is to be used. Substitution of the OPE between currents, Eq. (6.21), into Eq. 6.23) yields the desired result

$$
\left[Q_{a}, Q_{b}\right]_{ \pm}=f_{a b c} Q_{c}, \quad a, b=1, \cdots, 4
$$

We see that the first order poles in the OPE of the conserved current are determined solely by the symmetry group, and thus do not depend on the interaction. For the $U(1 / 1)$ symmetry, $f_{a b c}$ can be read off from the first order poles in Eq. (6.15). Moreover, we can generalize the calculation leading to Eq. (6.24) by introducing 


$$
Q_{a}^{f}(\tau)=\frac{1}{2 \pi \mathrm{i}} \int_{-\infty}^{+\infty} d x I_{a}(x, \tau) f(z), \quad a=1, \cdots, 4,
$$

for an arbitrary holomorhic function $f(z)$. We find

$$
\left[Q_{a}^{f}(\tau), I_{b}(x, \tau)\right]_{ \pm}=f^{\prime}(z) k_{a b}+f(z) f_{a b c} I_{c}(z), \quad a, b=1, \cdots, 4,
$$

or equivalently

$$
\left[I_{a}\left(x^{\prime}, \tau\right), I_{b}(x, \tau)\right]_{ \pm}=-\delta^{\prime}\left(x^{\prime}-x\right) k_{a b}+\delta\left(x^{\prime}-x\right) f_{a b c} I_{c}(z), \quad a, b=1, \cdots, 4 .
$$

To be consistent with the $U(1 / 1)$ symmetry, $k_{a b}$ must be an invariant tensor of the symmetry group. This constraint alone requires $k_{a b}$ to depend on only two (real) parameters $\left(k, k_{j}\right)$, which are uniquely determined by the $U(1 / 1)$ symmetric interaction. To see this, we consider first an infinitesimal transformation generated by $\bar{\theta} \eta+\theta \bar{\eta}$. Under this transformation, the currents $(J, j, \eta, \bar{\eta})$ change by the infinitesimal amounts

$$
\left(\delta_{\eta}+\delta_{\bar{\eta}}\right) J=0, \quad\left(\delta_{\eta}+\delta_{\bar{\eta}}\right) j=2 \bar{\eta} \theta+2 \bar{\theta} \eta, \quad\left(\delta_{\eta}+\delta_{\bar{\eta}}\right) \eta=\theta J, \quad\left(\delta_{\eta}+\delta_{\bar{\eta}}\right) \bar{\eta}=\bar{\theta} .
$$

Here, as a result of current conservation, the transformation law obeyed by the currents is independent of the interaction, and thus can be derived from the free theory $\mathcal{Z}_{0}$ of Eq. (6.1). Now, by requiring that $k_{a b}$ be invariant under $U(1 / 1)$, it follows that any infinitesimal change $\delta\left(I_{a}(z) I_{b}(0)\right)$ generated by the currents does not contain poles higher than first order in $z$. For example, from Eq. (6.27) one finds

$$
\begin{aligned}
\left(\delta_{\eta}+\delta_{\bar{\eta}}\right)(\eta(z) j(0)) & =\theta J(z) j(0)+2 \eta(z)(\bar{\eta}(0) \theta+\bar{\theta} \eta(0)) \\
& =\frac{\theta\left(k_{J j}+2 k_{\eta \bar{\eta}}\right)}{z^{2}}-\frac{2 \bar{\theta} k_{\eta \eta}}{z^{2}}+\mathcal{O}\left(\frac{1}{z}\right),
\end{aligned}
$$

which implies that $k_{J j}+2 k_{\eta \bar{\eta}}=0$ and $k_{\eta \eta}=0$. Repeating this argument for all $\delta\left(I_{a}(z) I_{b}(0)\right)$, we find that the most general OPE between the currents generating $U(1 / 1)$ transformations in the holomorphic sector are given by

$$
\begin{aligned}
& J(z) J(0)=0, \quad J(z) j(0)=\frac{2 k}{z^{2}}, \quad J(z) \eta(0)=0, \quad J(z) \bar{\eta}(0)=0, \\
& j(z) J(0)=\frac{2 k}{z^{2}}, \quad j(z) j(0)=\frac{k_{j}}{z^{2}}, \quad j(z) \eta(0)=-\frac{2}{z} \eta(0), \quad j(z) \bar{\eta}(0)=+\frac{2}{z} \bar{\eta}(0), \\
& \eta(z) J(0)=0, \quad \eta(z) j(0)=+\frac{2}{z} \eta(0), \quad \eta(z) \eta(0)=0, \quad \eta(z) \bar{\eta}(0)=-\frac{k}{z^{2}}+\frac{1}{z} J(0), \\
& \bar{\eta}(z) J(0)=0, \quad \bar{\eta}(z) j(0)=-\frac{2}{z} \bar{\eta}(0), \quad \bar{\eta}(z) \eta(0)=+\frac{k}{z^{2}}+\frac{1}{z} J(0), \quad \bar{\eta}(z) \bar{\eta}(0)=0 .
\end{aligned}
$$

We show in appendix $\mathrm{B}$ that $k=1, k_{j}>0$ corresponds to the current algebra derived from the effective partition function $\mathcal{Z}_{\mathrm{cr}}^{(1,0)}$ of Eq. (3.25) with $U(1)$ vector gauge-like disorder. In other words, the $U(1 / 1) \times U(1 / 1)$ current algebra given by Eq. (6.29) with $k=1, k_{j}>0$ describes a two-dimensional random $U(1)$ vector potential model at criticality. It is also shown that the OPE between the currents is self-consistent (associative) for all real values of $\left(k, k_{j}\right)$. In other words, all correlation functions for the currents are uniquely defined by their OPE in Eq. (6.29).

We close this subsection with the construction of the energy-momentum tensor associated with the OPE in Eq. (6.29). The holomorphic component $T$ of the energy-momentum tensor (EM) describes important properties of the fixed point. We sketch here how the energy-momentum tensor $T$ in the holomorphic sector is uniquely specified by the requirements that: $i) T$ is Hermitean and is solely constructed from the currents $I_{a}$, ii) $T$ has conformal weights $(2,0)$, iii) $T$ transforms like a singlet under $U(1 / 1)$. At the classical level, conditions $i)$ and $i i i)$ are met by the Ansatz

$$
T(z)=\sum_{a, b=1}^{4}\left(I_{a} T_{a b} I_{b}\right)(z),
$$

where $T_{a b}$ is Hermitean. The condition that $T$ be a $U(1 / 1)$ singlet is purely classical. Imposing $\left.i i i\right)$ yields

$$
T(z)=\kappa_{1}(J j+\eta \bar{\eta}-\bar{\eta} \eta)(z)+\kappa_{2}(J J)(z),
$$

where $\kappa_{1,2}$ are real. At the quantum level, conditions $i$ ) and $i$ ) must be treated with care. The quantum definition of $T$ involves taking the limit 


$$
\begin{aligned}
T(z) & =\lim _{\epsilon \rightarrow 0}\left\{\kappa_{1}\left[\frac{1}{2} J(z+\epsilon) j(z)+\frac{1}{2} j(z+\epsilon) J(z)+\eta(z+\epsilon) \bar{\eta}(z)-\bar{\eta}(z+\epsilon) \eta(z)\right]\right. \\
& \left.+\kappa_{2} J(z+\epsilon) J(z)\right\},
\end{aligned}
$$

which defines the product of operators at the same point. In general, we need to subtract out some infinities, i.e., the poles that appear in the OPE of the currents, in order for the EM tensor to be finite in the limit $\epsilon \rightarrow 0$. However, one can show from the OPE in Eq. (6.29) that $T$, as defined by Eq. (6.32), is already finite so that additional subtractions are not needed. Also note that the right-hand side of Eq. (6.32) is a $U(1 / 1)$ singlet even for finite values of $\epsilon$. The two additional coefficients $\kappa_{1}$ and $\kappa_{2}$ can be determined from the OPE of the EM tensor. In a two-dimensional conformal field theory [40], the OPE of the EM tensor must have the form

$$
T(z) T(0)=\frac{\frac{c}{2}}{z^{4}}+\frac{2}{z^{2}} T(0)+\frac{1}{z} \partial_{z} T(0) .
$$

The residue of the fourth order pole determines the so-called Virasoro central charge $c$. The residue of the second order pole determines the conformal weight of the energy momentum tensor, namely two. The constraint $i i)$ is equivalent to imposing Eq. (6.33). One can show from the OPE in Eq. (6.29) and the definition Eq. (6.32) that Eq. (6.33) holds if

$$
\kappa_{1}=\frac{1}{2 k}, \quad \kappa_{2}=\frac{4-k_{j}}{8 k^{2}}
$$

in which case the Virasoro central charge vanishes: $c=0$. One can also show that the currents $(J, j, \eta, \bar{\eta})$ have conformal weights $(1,0)$, since their OPE with $T$ is given by:

$$
T(z) I_{a}(0)=\frac{1}{z^{2}} I_{a}(0)+\frac{1}{z} \partial_{z} I_{a}(0), \quad a=1, \cdots, 4
$$

Conversely, we show in appendix A that, requiring Eq (6.35) to hold together with the definition Eq (6.32), uniquely defines the energy momentum tensor.

\section{Operators in one- and two-dimensional representations of the $U(1 / 1)$ Lie algebra}

The $U(1 / 1)$ current algebra contains many operators apart from the four currents $(J, j, \eta, \bar{\eta})$. For example, from the fact that the current $J$ is a singlet of $U(1 / 1)$, we can use $J$ to construct operators which form a one-dimensional representation of the $U(1 / 1)$ Lie algebra. Let us consider the following operator

$$
f_{q}(z) \equiv e^{-\mathrm{i} \frac{q}{2 k} \theta_{J}(z)}
$$

where $q$ is real, and $\theta_{J}(z)$ is the solution of

$$
\partial_{z} \theta_{J}(z) \equiv \mathrm{i} J(z)
$$

We use the OPE between the currents to construct the $U(1 / 1)$ charges of $f_{q}$ and its (holomorphic) conformal weight $h_{f_{q}}$. One verifies that $f_{q}(z)$ has the following OPE with the currents

$$
J(z) f_{q}(0)=0, \quad j(z) f_{q}(0)=-\frac{q}{z} f_{q}(0), \quad \eta(z) f_{q}(0)=\bar{\eta}(z) f_{q}(0)=0
$$

The OPE in Eq. 6.37) gives the $U(1 / 1)$ charges $(J, j, \eta, \bar{\eta})=(0,-q, 0,0)$ of $f_{q}$. Clearly, $f_{q}^{\dagger} f_{q}$ is then a $U(1 / 1)$ singlet, and $f_{q}$ carries a one-dimensional representation of $U(1 / 1)$. The OPE in Eq. (6.37) allows us to reduce any correlations between currents and $f_{q}$ 's into one that involve only $f_{q}$ 's. The correlation between $f_{q}$ 's is given by

$$
\left\langle f_{q}\left(z_{1}\right) f_{-q}\left(z_{2}\right)\right\rangle=\text { constant }
$$

since $J(z) J(0)=0$. This implies that $f_{q}$ has vanishing conformal weight: $h_{f_{q}}=0$. More generally, the $n$-point correlation function for $f_{q}$ is given by 


$$
\left\langle\prod_{i=1}^{n} f_{q_{i}}\left(z_{i}\right)\right\rangle= \begin{cases}0 & \text { if } \sum_{i=1}^{n} q_{i}, \neq 0, \\ \text { constant } & \text { if } \sum_{i=1}^{n} q_{i}=0 .\end{cases}
$$

The $U(1 / 1)$ current algebra also contains operators which form two-dimensional representations of the $U(1 / 1)$ algebra. To see this, we define

$$
\psi_{p}^{\dagger} \equiv \varphi^{\dagger(p-1)} \psi^{\dagger}, \quad \varphi_{p}^{\dagger} \equiv \frac{1}{\sqrt{p}} \varphi^{\dagger p}, \quad \psi_{p} \equiv \psi \varphi^{(p-1)}, \quad \varphi_{p} \equiv \frac{1}{\sqrt{p}} \varphi^{p},
$$

where $p$ is a positive integer. By showing that $\psi_{p}^{\dagger} \psi_{p}+\varphi_{p}^{\dagger} \varphi_{p}$ is a singlet under $U(1 / 1)$, it follows that $\left(\psi_{p}, \varphi_{p}\right)$ transforms like a doublet of $U(1 / 1)$. Notice that this is certainly true when $p=1$. The $J$ and $j$ charges of $\left\{\psi_{p}^{\dagger}, \varphi_{p}^{\dagger}, \psi_{p}, \varphi_{p}\right\}$ can be read from Eq. (6.40). They are $\{p, p,-p,-p\}$ and $\{2-p,-p, p-2, p\}$, respectively. Hence, on the one hand, the $J$ and $j$ charges of $\psi_{p}^{\dagger} \psi_{p}$ and $\varphi_{p}^{\dagger} \varphi_{p}$ both vanish. On the other hand, because of the infinitesimal transformation law

$$
\begin{aligned}
& \delta_{\eta} \psi_{p}^{\dagger}=+\sqrt{p} \varphi_{p}^{\dagger} \bar{\theta}, \quad \delta_{\eta} \varphi_{p}^{\dagger}=0, \quad \delta_{\eta} \psi_{p}=0, \quad \delta_{\eta} \varphi_{p}=-\sqrt{p} \bar{\theta} \psi_{p}, \\
& \delta_{\bar{\eta}} \psi_{p}^{\dagger}=0, \quad \delta_{\bar{\eta}} \varphi_{p}^{\dagger}=-\sqrt{p} \psi_{p}^{\dagger} \theta, \quad \delta_{\bar{\eta}} \psi_{p}=+\sqrt{p} \bar{\theta} \psi_{p}, \quad \delta_{\bar{\eta}} \varphi_{p}=0,
\end{aligned}
$$

which is generated by the fermionic currents $\eta$, and $\bar{\eta}$ of $U(1 / 1)$ (compare with Eq. (6.7)), only the sum of $\psi_{p}^{\dagger} \psi_{p}$ and $\varphi_{p}^{\dagger} \varphi_{p}$ is a $U(1 / 1)$ singlet. Moreover, we can read from Eq. 6.41) and the $J$ and $j$ charges of $\psi_{p}^{\dagger}, \varphi_{p}^{\dagger}, \psi_{p}, \varphi_{p}$, the OPE between the currents and $\left\{\psi_{p}^{\dagger}, \varphi_{p}^{\dagger}, \psi_{p}, \varphi_{p}\right\}$. They are

$$
\begin{aligned}
& J(z) \psi_{p}^{\dagger}(0)=\frac{p}{z} \psi_{p}^{\dagger}(0), j(z) \psi_{p}^{\dagger}(0)=\frac{2-p}{z} \psi_{p}^{\dagger}(0), \eta(z) \psi_{p}^{\dagger}(0)=\frac{\sqrt{p}}{z} \varphi_{p}^{\dagger}(0), \bar{\eta}(z) \psi_{p}^{\dagger}(0)=0, \\
& J(z) \varphi_{p}^{\dagger}(0)=\frac{p}{z} \varphi_{p}^{\dagger}(0), j(z) \varphi_{p}^{\dagger}(0)=-\frac{p}{z} \varphi_{p}^{\dagger}(0), \eta(z) \varphi_{p}^{\dagger}(0)=0, \bar{\eta}(z) \varphi_{p}^{\dagger}(0)=-\frac{\sqrt{p}}{z} \psi_{p}^{\dagger}(0), \\
& J(z) \psi_{p}(0)=-\frac{p}{z} \psi_{p}(0), j(z) \psi_{p}(0)=-\frac{2-p}{z} \psi_{p}(0), \eta(z) \psi_{p}(0)=0, \bar{\eta}(z) \psi_{p}(0)=\frac{\sqrt{p}}{z} \varphi_{p}(0), \\
& J(z) \varphi_{p}(0)=-\frac{p}{z} \varphi_{p}(0), j(z) \varphi_{p}(0)=\frac{p}{z} \varphi_{p}(0), \eta(z) \varphi_{p}(0)=-\frac{\sqrt{p}}{z} \psi_{p}(0), \bar{\eta}(z) \varphi_{p}(0)=0 .
\end{aligned}
$$

We see that the OPE of (3.37) and (3.38) are well defined even when $p$ is not an integer. From now on we will regard $p$ to be a positive real number.

Even more general doublet can be constructed if we combine the $\psi_{p}^{\dagger}, \cdots, \varphi_{p}$ with $f_{q}$ by defining

$$
\psi_{p q}^{\dagger} \equiv f_{q}^{\dagger} \psi_{p}^{\dagger}, \quad \varphi_{p q}^{\dagger} \equiv f_{q}^{\dagger} \varphi_{p}^{\dagger}, \quad \psi_{p q} \equiv \psi_{p} f_{q}, \quad \varphi_{p q} \equiv \varphi_{p} f_{q} .
$$

Here, $p$ is a positive real number while $q$ is real. The OPE between $\left(\psi_{p q}, \varphi_{p q}\right)$ and the currents are very similar to those between $\left(\psi_{p}, \varphi_{p}\right)$ and the currents, since the multiplication of $f_{q}$ merely shifts the quantum number of $j$. In fact, the OPE between $\left(\psi_{p q}, \varphi_{p q}\right)$ and the currents are still given by Eq. (6.42) except for $p$ in the OPE with the current $j$ being shifted to $p-q$. In fact, the transformation law of Eq. (6.41) also applies to $\left(\psi_{p q}, \varphi_{p q}\right)$.

From the OPE of Eqs. (6.29,6.37,6.42), together with the definition of the EM tensor in Eq. (6.32), we can calculate the OPE between $T$ and $\left(\psi_{p q}, \varphi_{p q}\right)$. It is

$$
\begin{aligned}
T(z) \psi_{p q}(0) & =\frac{h_{p q}}{z^{2}} \psi_{p q}(0)+\frac{1}{z}\left(\partial_{z} \psi_{p q}\right)(0), \\
T(z) \varphi_{p q}(0) & =\frac{h_{p q}}{z^{2}} \varphi_{p q}(0)+\frac{1}{z}\left(\partial_{z} \varphi_{p q}\right)(0) .
\end{aligned}
$$

Eq. (6.44) implies that $\left(\psi_{p q}, \varphi_{p q}\right)$ are primary fields [40] with (holomorphic) conformal weight given by

$$
h_{p q}=\frac{p}{2 k}(1-p+q)+\frac{p^{2}}{2 k^{2}}\left(1-\frac{k_{j}}{4}\right) .
$$

For the free theory of Eq. (6.1), $\left(k, k_{j}\right)=(1,0)$. In this case Eq. 6.45) predicts $h_{p 0}=\frac{p}{2}$, which agrees with the dimension of $\psi \varphi^{p-1}$ and $\varphi^{p}$ calculated using the dimension $h_{\psi, \varphi}=\frac{1}{2}$. Similarly, $\left(\psi_{p q}^{\dagger}, \varphi_{p q}^{\dagger}\right)$ have the same scaling dimension $h_{p q}$. This is a consequence of the charge conjugation symmetry of the theory. 
We have shown that the OPE between the currents in Eq. (6.29) completely determine all their $n$-point correlation functions. We want to know if the same holds for the doublets $\left(\psi_{p q}, \varphi_{p q}\right)$, i.e., whether it is possible to calculate any $n$-point correlation function between those new operators and the currents. We begin with the two-point correlation functions between $\psi_{p q}^{\dagger}, \varphi_{p q}^{\dagger}, \psi_{p q}, \varphi_{p q}$. On the one hand, from Eq. (6.42), $\psi_{p q} \psi_{p q}^{\dagger}$ and $\varphi_{p q} \varphi_{p q}^{\dagger}$ have vanishing $(J, j)$ charges, and, therefore, can acquire non-vanishing expectation values. We see that current conservation together with Eq. (6.44) requires that

$$
\left\langle\psi_{p q}(z) \psi_{p q}^{\dagger}(0)\right\rangle=\frac{C_{\psi}}{z^{2 h_{p q}}}, \quad\left\langle\varphi_{p q}(z) \varphi_{p q}^{\dagger}(0)\right\rangle=\frac{C_{\varphi}}{z^{2 h_{p q}}} .
$$

On the other hand, Eq. (6.42) tells us that $\psi_{p q} \varphi_{p q}^{\dagger}$ and $\varphi_{p q} \psi_{p q}^{\dagger}$ have the $(J, j)$ charges $(0,-2)$ and $(0,+2)$, respectively. Hence, they cannot acquire non-vanishing expectation values due to current conservation:

$$
\left\langle\psi_{p q}(z) \varphi_{p q}^{\dagger}(0)\right\rangle=\left\langle\varphi_{p q}(z) \psi_{p q}^{\dagger}(0)\right\rangle=0 .
$$

This allows us to relate the real parameters $C_{\psi}$ and $C_{\varphi}$. Indeed, if we use the transformation law

$$
\begin{aligned}
& \delta_{\eta} \psi_{p q}^{\dagger}=+\sqrt{p} \varphi_{p q}^{\dagger} \bar{\theta}, \quad \delta_{\eta} \varphi_{p q}^{\dagger}=0, \quad \delta_{\eta} \psi_{p q}=0, \quad \delta_{\eta} \varphi_{p q}=-\sqrt{p} \bar{\theta} \psi_{p q}, \\
& \delta_{\bar{\eta}} \psi_{p q}^{\dagger}=0, \quad \delta_{\bar{\eta}} \varphi_{p q}^{\dagger}=-\sqrt{p} \psi_{p q}^{\dagger} \theta, \quad \delta_{\bar{\eta}} \psi_{p q}=+\sqrt{p} \bar{\theta} \psi_{p q}, \quad \delta_{\bar{\eta}} \varphi_{p q}=0,
\end{aligned}
$$

under infinitesimal transformations generated by $\eta$, and $\bar{\eta}$, we can show that

$$
\left(\delta_{\eta}+\delta_{\bar{\eta}}\right)\left\langle\varphi_{p q}(z) \psi_{p q}^{\dagger}(0)\right\rangle=\sqrt{p} \bar{\theta}\left(-C_{\psi}+C_{\varphi}\right) \frac{1}{z^{2 h_{p q}}} .
$$

Eqs. (6.47) and (6.49) then imply that $C_{\psi}=C_{\varphi}$. Setting $C_{\psi}=C_{\varphi}=1$ through rescaling of the doublet $\left(\psi_{p q}, \varphi_{p q}\right)$, reduces Eq. (6.46) to

$$
\left\langle\psi_{p q}(z) \psi_{p q}^{\dagger}(0)\right\rangle=\left\langle\varphi_{p q}(z) \varphi_{p q}^{\dagger}(0)\right\rangle=\frac{1}{z^{2 h_{p q}}} .
$$

It is useful to consider the OPE of two doublets $\left(\psi_{p_{1} q_{1}}, \varphi_{p_{1} q_{1}}\right)$ and $\left(\psi_{p_{2} q_{2}}, \varphi_{p_{2} q_{2}}\right)$. One can easily see that the product $\left(\psi_{p_{1} q_{1}}, \varphi_{p_{1} q_{1}}\right) \times\left(\psi_{p_{2} q_{2}}, \varphi_{p_{2} q_{2}}\right)$ contains a new doublet $\left(\psi_{p_{3} q_{3}}, \varphi_{p_{3} q_{3}}\right)$ with $p_{3}=p_{1}+p_{2}$ and $q_{3}=q_{1}+q_{2}$. Thus, the OPE of $\left(\psi_{p_{1} q_{1}}, \varphi_{p_{1} q_{1}}\right)$ and $\left(\psi_{p_{2} q_{2}}, \varphi_{p_{2} q_{2}}\right)$ must be of the form

$$
\begin{aligned}
\varphi_{p_{1} q_{1}}(z) \varphi_{p_{2} q_{2}}(0) & \sim \frac{1}{z^{h_{1}+h_{2}-h_{3}}} \varphi_{p_{3} q_{3}}+\cdots, \\
\psi_{p_{1} q_{1}}(z) \varphi_{p_{2} q_{2}}(0) & \sim \frac{1}{z^{h_{1}+h_{2}-h_{3}}} \psi_{p_{3} q_{3}}+\cdots, \\
\varphi_{p_{1} q_{1}}(z) \psi_{p_{2} q_{2}}(0) & \sim \frac{1}{z^{h_{1}+h_{2}-h_{3}}} \psi_{p_{3} q_{3}}+\cdots,
\end{aligned}
$$

where $h_{i}$ are dimensions of $\left(\psi_{p_{i} q_{i}}, \varphi_{p_{i} q_{i}}\right)$. This formula will be used below to discuss the locality of operators.

\section{Local operators and quantization of $k$}

According to the general principles of two-dimensional conformal field theory (CFT) 40], any generic operator can be written as the product of operators in the holomorphic $(z)$ and antiholomorphic $(\bar{z})$ sectors:

$$
O(z, \bar{z})=O_{z}(z) O_{\bar{z}}(\bar{z}) .
$$

The OPE of such mixed operators are given by

$$
O_{a}(z, \bar{z}) O_{b}(0,0)=\sum_{c} C_{a b}^{c} z^{h_{c}-h_{a}-h_{b}} \bar{z}^{\bar{h}_{c}-\bar{h}_{a}-\bar{h}_{b}} O_{c}(0,0)
$$

where $\left(h_{a}, \bar{h}_{a}\right)$ are the conformal weights of $O_{a}$, and $C_{a b}^{c}$ are complex numbers.

The concept of a local operator is very important in our theory. In CFT, two operators $O_{a}(z, \bar{z})$ and $O_{b}(z, \bar{z})$ are said to be mutually local if their OPE (i.e., the right-hand side of Eq. (6.53)) is single-valued. This implies that the spin difference 


$$
s_{c}-s_{a}-s_{b} \equiv\left(h_{c}-\bar{h}_{c}\right)-\left(h_{a}-\bar{h}_{a}\right)-\left(h_{b}-\bar{h}_{b}\right)
$$

is an integer for all $c$ with non-vanishing $C_{a b}^{c}$. A set of operators is said to be made of local operators if they are all pairwise mutually local. We will call the set of all local operators the "center" of the operator product algebra. From this definition we see that the correlation functions between local operators (i.e., between operators in the center of the operator product algebra) are always single-valued functions.

With these definitions in hand, we come back to the theory defined by Eq. (6.20), assuming only that the interaction be invariant with respect to $U(1 / 1)$. In the presence of a generic $U(1 / 1)$ symmetric interaction $\mathcal{L}_{\text {int }}^{U(1 / 1)}$, the spatial dependency of the doublets $\left(\psi_{+}, \varphi_{+}\right)$and $\left(\psi_{-}, \varphi_{-}\right)$are no longer purely holomorphic and antiholomorphic, respectively. This is not to say that the holomorphic and antiholomorphic sectors are absent. Any critical point of Eq. (6.20) with $U(1 / 1)$ symmetry still possesses two decoupled sectors characterized by holomorphic and antiholomorphic correlation functions. Rather, the doublets $\left(\psi_{ \pm}, \varphi_{ \pm}\right)$are not necessarily primary fields anymore. Instead, they are of the mixed form in Eq. (6.52). (They do, however, still transform like $U(1 / 1)$ doublets due to current conservation.)

It should be obvious that $\psi_{+}^{\dagger}, \cdots, \varphi_{-}$and their products in our model Eq. (6.20) are local operators provided we assume that the interaction $\mathcal{L}_{\text {int }}^{U(1 / 1)}$, besides being $U(1 / 1)$ symmetric, is also local (i.e., only involves product of fields at the same point). If so, the doublets $\left(\psi_{ \pm}, \varphi_{ \pm}\right)$and $\left(\psi_{ \pm}^{\dagger}, \varphi_{ \pm}^{\dagger}\right)$ are local regardless of whether the model is at a critical point or away from a critical point and regardless of the nature of the critical point as long as it possesses the $U(1 / 1)$ symmetry. Thus, any critical point associated to $\mathcal{L}_{\text {int }}^{U(1 / 1)}$ must contain a set of local operators that carry the same $U(1 / 1)$ quantum numbers as $\psi_{ \pm}^{\dagger}, \varphi_{ \pm}^{\dagger}, \psi_{ \pm}, \varphi_{ \pm}$and their products do. We recall that $U(1 / 1)$ denotes the diagonal subgroup of $U(1 / 1) \times U(1 / 1)$, which appears at the critical point. This puts a strong constraint on the possible critical points that $\mathcal{L}_{\text {int }}^{U(1 / 1)}$ can have. We now show that the constraints of locality and of current conservation restricts the possible values of the central charge $k$ to be the inverse of some integer $m$ :

$$
k=\frac{1}{m}, \quad m \in \mathbf{Z}
$$

We also show that all the critical points with $U(1 / 1) \times U(1 / 1)$ symmetry for which $\psi_{+}^{\dagger}, \cdots, \varphi_{-}$are local are labelled by two integers $l, m \in \mathbf{Z}$ and one real number $k_{j}$.

The proof uses the components

$$
J_{\tau}=\mathrm{i}\left(J_{z}-J_{\bar{z}}\right), \quad j_{\tau}=\mathrm{i}\left(j_{z}-j_{\bar{z}}\right),
$$

which were defined in Eq. (6.8). Here, $J_{\tau}$ and $j_{\tau}$ are the bosonic generators of the diagonal subgroup $U(1 / 1)$ of $U(1 / 1) \times U(1 / 1)$. For convenience, we have listed some $U(1 / 1) \times U(1 / 1)$ charges in table $\mathbb{f}$ from which one immediately finds that the $J_{\tau}$ charges of $\left(\psi_{+}, \varphi_{+}, \psi_{-}, \varphi_{-}\right)$are $\mathrm{i}(-1,-1,1,1)$, while their $j_{\tau}$ charges are $\mathrm{i}(-1,1,1,-1)$. Let us assume that we are at a critical point with $U(1 / 1) \times U(1 / 1)$ symmetry characterized by the real valued KacMoody central charges $k$ and $k_{j}$. We know from the previous subsection that there exists primary fields $f_{q z}$ and $f_{q \bar{z}}$ ( $q$ real) with vanishing conformal weights and carrying a one-dimensional representation of the $U(1 / 1)$ Kac-Moody algebra in the holomorphic $(z)$ and antiholomorphic $(\bar{z})$ sectors, respectively. There also exists primary fields $\psi_{p q z}, \varphi_{p q z}$ and $\psi_{p q \bar{z}}, \varphi_{p q \bar{z}}\left(p\right.$ real and positive) carrying conformal weights $\left(h_{p q}, 0\right)$ and $\left(0, h_{p q}\right)$ (see Eq. (6.45)), respectively. Moreover, $\left(\psi_{p q z}, \varphi_{p q z}\right)$ transforms like a doublet (singlet) of the $U(1 / 1)$ Kac-Moody algebra in the holomorphic (antiholomorphic) sector, while $\left(\psi_{p q \bar{z}}, \varphi_{p q \bar{z}}\right)$ transforms like a doublet (singlet) of the $U(1 / 1)$ Kac-Moody algebra in the antiholomorphic (holomorphic) sector. Because $\left(\psi_{+}, \varphi_{+}\right)$and $\left(\psi_{-}, \varphi_{-}\right)$transform like doublets under diagonal $U(1 / 1)$, we must then have

$$
\left(\begin{array}{l}
\psi_{+}(z, \bar{z}) \\
\varphi_{+}(z, \bar{z})
\end{array}\right) \sim\left(\begin{array}{l}
\psi_{p q z}(z) f_{q^{\prime} \bar{z}}(\bar{z}) \\
\varphi_{p q z}(z) f_{q^{\prime} \bar{z}}(\bar{z})
\end{array}\right), \quad\left(\begin{array}{l}
\psi_{-}(z, \bar{z}) \\
\varphi_{-}(z, \bar{z})
\end{array}\right) \sim\left(\begin{array}{l}
\psi_{p q \bar{z}}(\bar{z}) f_{q^{\prime} z}(z) \\
\varphi_{p q \bar{z}}(\bar{z}) f_{q^{\prime} z}(z)
\end{array}\right)
$$

where $\sim$ means obeying the same transformation law under diagonal $U(1 / 1)$. The real numbers $p>0, q, q^{\prime}$ are thus not arbitrary, since the $J_{\tau}, j_{\tau}, \eta_{\tau}, \bar{\eta}_{\tau}$ charges of the left-hand side must equal those of the right-hand side. From Eq. (6.56) and table If conservation of the $J_{\tau}$ current yields the constraint

$$
\left(\begin{array}{l}
-1 \\
-1
\end{array}\right)=\left(\begin{array}{l}
-p \\
-p
\end{array}\right)
$$

while conservation of the $j_{\tau}$ current yields

$$
\left(\begin{array}{l}
-1 \\
+1
\end{array}\right)=\left(\begin{array}{c}
p-2-q+q^{\prime} \\
p-q+q^{\prime}
\end{array}\right)
$$


Current conservation fixes $p=1$ and only leaves $q=q^{\prime}$ undetermined. We thus have found that

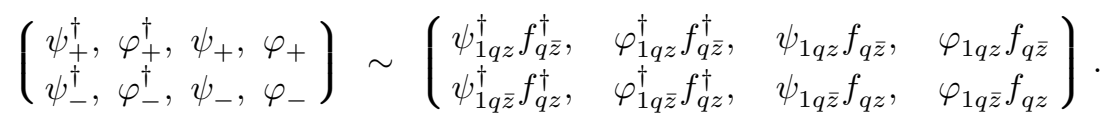

Again, $\sim$ means obeying the same transformation law under diagonal $U(1 / 1)$. Having derived the correlation functions of the primary fields on the right-hand side of Eq. (6.60), the correlation functions among $\left\{\psi_{+}^{\dagger}, \cdots, \varphi_{-}\right\}$can be calculated. But since $\left\{\psi_{+}^{\dagger}, \cdots, \varphi_{-}\right\}$are local operators, their correlation functions must be single-valued. The condition of locality then further limits the real valued $q$. For example,

$$
\left\langle\varphi_{+}(z, \bar{z}) \varphi_{+}^{\dagger}(0,0)\right\rangle=z^{-2 h_{1 q}}
$$

is only single-valued if

$$
2 h_{1 q}=l,
$$

where $l$ is an integer. In other words, using Eq. (6.45) we find that $q$ is parameterized by an integer $l$

$$
q=l k-\frac{1}{k}\left(1-\frac{k_{j}}{4}\right), \quad l \in \mathbf{Z} .
$$

One can verify that Eq. (6.63) insures that all correlation functions among $\left\{\psi_{+}^{\dagger}, \cdots, \varphi_{-}\right\}$and powers thereof are single-valued.

Finally, we show that the constraint of locality on the OPE among $\left\{\psi_{+}^{\dagger}, \cdots, \varphi_{-}\right\}$forces the Kac-Moody central charge $k$ to be quantized. Without loss of generality, we only consider the OPE between $\varphi_{+}(z, \bar{z})$ and $\varphi_{+}(0,0)$. Except for the residues, it is the same as the OPE between $\varphi_{1 q z}(z)$ and $\varphi_{1 q z}(0)$ due to Eq. (6.39). It must therefore be of the form (see Eq. (6.51)):

$$
\varphi_{+}(z, \bar{z}) \varphi_{+}(0,0)=z^{h_{2(2 q)}-2 h_{1 q}} \varphi_{2(2 q) z}(0) f_{(2 q) \bar{z}}(0)+\cdots .
$$

Locality again requires that

$$
h_{2(2 q)}-2 h_{1 q}=l+\frac{1}{k}
$$

be an integer, i.e., $k$ must be the inverse of some integer $m \in \mathbf{Z}$. We thus see that the $U(1 / 1)$ critical point of $\mathcal{L}_{\text {int }}^{U(1 / 1)}$ must be labelled by the triplet $\left(l, m, k_{j}\right)$ with $l, m \in \mathbf{Z}$ and $k_{j}$ being a real number.

\section{E. Operator content of the $U(1 / 1) \times U(1 / 1)$ critical points}

We assume that we are at the critical point labelled by the triplet $\left(l, m, k_{j}\right)$. The full local operator content of this critical point can be obtained from the operator algebra generated by the currents $(J, j, \eta, \bar{\eta})$ and the local operators $\left\{\psi_{+}^{\dagger}, \cdots, \varphi_{-}\right\}$. In the following we want to calculate the conformal weights of some local composite operators. We begin with

$$
\Phi_{+n}=\varphi_{+}^{|n|}, \quad \Phi_{-n}=\varphi_{-}^{|n|}, \quad n \in \mathbf{Z} .
$$

With a calculation along the lines of the previous subsection, one verifies that the conformal weights $(h, 0)$ of $\Phi_{+n}$ are the same as the conformal weights $\left(h_{p^{\prime} q^{\prime}}, 0\right)$ of $\varphi_{p^{\prime} q^{\prime} z}$, where $p^{\prime}=n, q^{\prime}=n q$. Hence, with the help of Eqs. (6.55), (6.45) and (6.63),

$$
h=\frac{1}{2} m|n|+\frac{1}{2} n^{2}(l-m), \quad n \in \mathbf{Z} .
$$

Similarly, $\Phi_{-n}$ has $(0, h)$ for conformal weights. More generally, we introduce (compare with Eq. (3.30a))

$$
\Phi_{n_{1} n_{2}}= \begin{cases}\varphi_{+}^{n_{1}} \varphi_{-}^{n_{2}}, & n_{1}>0, n_{2}>0, \\ \varphi_{+}^{\dagger-n_{1}} \varphi_{-}^{n_{2}}, & n_{1}<0, n_{2}>0, \\ \varphi_{+}^{n_{1}} \varphi_{-}^{\dagger-n_{2}}, & n_{1}>0, n_{2}<0, \\ \varphi_{+}^{\dagger-n_{1}} \varphi_{-}^{\dagger-n_{2}}, & n_{1}<0, n_{2}<0\end{cases}
$$


where $n_{1}, n_{2}$ are integers. The conformal weights $(h, \bar{h})$ of $\Phi_{n_{1} n_{2}}$ are equal to: $(i)$ those of $\varphi_{p^{\prime} q^{\prime} z}$ in the holomorphic sector with $p^{\prime}=\left|n_{1}\right|, q^{\prime}=\left(\left|n_{1}\right| \pm\left|n_{2}\right|\right) q$, and $(i i)$ those of $\varphi_{p^{\prime} q^{\prime}} \bar{z}$ in the antiholomorphic sector with $p^{\prime}=\left|n_{2}\right|, q^{\prime}=$ $\left(\left|n_{2}\right| \pm\left|n_{1}\right|\right) q$. Thus, according to Eqs. (6.55), 6.45) and (6.63)

$$
\begin{aligned}
& h=\frac{1}{2} m\left|n_{1}\right|+\frac{1}{2} n_{1}^{2}(l-m)+\frac{1}{2} n_{1} n_{2}\left[l-m^{2}\left(1-\frac{k_{j}}{4}\right)\right], \\
& \bar{h}=\frac{1}{2} m\left|n_{2}\right|+\frac{1}{2} n_{2}^{2}(l-m)+\frac{1}{2} n_{1} n_{2}\left[l-m^{2}\left(1-\frac{k_{j}}{4}\right)\right] .
\end{aligned}
$$

Hence, the scaling dimension $\Delta_{n_{1} n_{2}}=h+\bar{h}$ and spin $s_{n_{1} n_{2}}=h-\bar{h}$ of $\Phi_{n_{1} n_{2}}$ for $n_{1}, n_{2} \in \mathbf{Z}$ are

$$
\begin{aligned}
\Delta_{n_{1} n_{2}} & =\frac{1}{2} m\left(\left|n_{1}\right|+\left|n_{2}\right|\right)+\frac{1}{2}\left(n_{1}^{2}+n_{2}^{2}\right)(l-m)+n_{1} n_{2}\left[l-m^{2}\left(1-\frac{k_{j}}{4}\right)\right], \\
s_{n_{1} n_{2}} & =\frac{1}{2} m\left(\left|n_{1}\right|-\left|n_{2}\right|\right)+\frac{1}{2}\left(n_{1}^{2}-n_{2}^{2}\right)(l-m),
\end{aligned}
$$

respectively. Notice that we recover the conformal weights of Eq. (3.30b) if $k_{j}=\frac{4 g_{A}}{\pi}$.

We see from Eqs. (6.67,6.70b) that the spins of $\Phi_{+n}, \Phi_{-n}$, and $\Phi_{n_{1} n_{2}}$ are either integers or half-integers depending on whether the labels $l, m \in \mathbf{Z}$ of the critical point are even or odd. The composite operators $\Phi_{+n}, \Phi_{-n}$, and $\Phi_{n_{1} n_{2}}$ are thus local for all integer valued $n, n_{1}$ and $n_{2}$, according to Eq. (6.54).

It should be noted that for any critical point $\left(l, m, k_{j}\right)$, the condition that $\psi_{ \pm}$and $\varphi_{ \pm}$have half-integer spin (although not necessarily $\left.\frac{1}{2}\right)$ must hold. This is so because the transformation corresponding to a rotation in the $(\tau, x)$ plane by $2 \pi$ changes the sign of the spinors $\psi_{ \pm}$and $\varphi_{ \pm}$, while it leaves the impurity potential unchanged. Hence, this property should be preserved at the interacting fixed point. It then follows from Eq. (6.70b) that $l$ is an odd integer. Other constraints on the possible critical points $\left(l, m, k_{j}\right)$ require additional symmetries. For example by assuming that the interaction $\mathcal{L}_{\text {int }}^{U(1 / 1)}$ is invariant under rotations of the Euclidean plane $(x, \tau)$, it follows that only $l=1$ is allowed. Indeed, at the free theory critical point $(1,1,0), \psi_{ \pm}$and $\varphi_{ \pm}$have spin $\pm \frac{1}{2}$. By assumption, this quantum number is unchanged at the new critical point $\left(l, m, k_{j}\right)$. From Eq. 6.70b), the condition that, say $s_{10}=\frac{1}{2}$, thus requires $l=1$.

In summary, the possible critical points $\mathcal{L}_{0}+\mathcal{L}_{\text {int }}^{U(1 / 1)}$ with $U(1 / 1) \times U(1 / 1)$ symmetry are labelled by the odd integer $l$, the integer $m$ and a real number $k_{j}:\left(l, m, k_{j}\right)$. Many correlation functions can be calculated exactly from the OPE. Although $\left(l, m, k_{j}\right)$ enumerates all possible critical points with currents obeying a $U(1 / 1) \times U(1 / 1)$ KacMoody algebra, it is not clear whether or not all these critical points can be realized by a theory with a Lagrangian solely constructed from the fermionic spinors $\psi_{ \pm}^{\dagger}, \psi_{ \pm}$and the bosonic spinors $\varphi_{ \pm}^{\dagger}, \varphi_{ \pm}$. We were only able to identify the line $\left(1,1, k_{j}\right), k_{j}>0$, with a Lagrangian realization of the random $U(1)$ vector gauge-like model. Finally, the same approach can be used to identify all critical points with currents obeying the $G \times G$ Kac-Moody algebra, where $G$ is a graded Lie algebra. This we have done explicitly for the compact group $U(2 / 2)$ and the non-compact group $U(1,1 / 1,1)$. In both cases, all critical points are labelled by the triplet $\left(l, m, k_{j}\right)$ and Eq. (6.69) holds.

\section{DISCUSSION}

We have studied a model of massless Dirac fermions in two spatial dimensions and in the presence of static random vector potentials which are distributed according to a white noise probability distribution. We have shown that this model is an example of a non-unitary two-dimensional CFT with a highly unusual operator content, since there exists an infinite hierarchy of negative dimensional operators. The physical origin of this hierarchy of negative dimensional operators is the fact that the single-particle Green function is log-normal distributed with respect to realizations of the randomness. In turn, the non-selfaveraging nature of the single-particle Green function is related to the multifractal nature of a typical wave function with vanishing energy, although it is still an open question of how to calculate the multifractal scaling exponents associated to the critical (i.e., vanishing energy) typical (i.e., for a fixed realization of the disorder) wave function from the field theory. We have argued that such disordered critical points are unstable in a very special way, if perturbations corresponding to, say, randomness in the mass are present in the lattice regularization of Dirac fermions. Indeed, in addition to the strength of the mass-like randomness, the shape of its probability distribution induces infinitely many relevant perturbations through all the cumulants of the probability distribution.

On the one hand, it would appear from our study of disordered critical points with $U(1 / 1) \times U(1 / 1)$ graded Kac-Moody algebra that the existence of infinite hierarchies of negative dimensional operators is related to the 
multifractality of a typical critical wave function, and leads to a very special instability of the critical point. On the other hand, there are numerical evidences that the plateau transition in the integer quantum Hall effect is also characterized by negative dimensional operators [7]. If so, they clearly do not destabilize the disordered critical point corresponding to the plateau transition. The most obvious difference between the plateau transition and Dirac fermions with random vector potentials is that the averaged density of states is non-critical in the former. This is very suggestive of requiring spontaneous symmetry breaking of the chiral symmetry of disordered Dirac fermions (there is no Mermin-Wagner theorem preventing this from happening in the context of a non-unitary CFT). Hence, it might be too restrictive to assume as we did that there is no spontaneous symmetry breaking of the $U(1 / 1)$ graded symmetry.

A disordered system related to Dirac fermions in the presence of random vector potentials is the two-dimensional $X Y$ model with quenched random bond coupling studied by Rubinstein et al. [59]. Indeed, one can use the equivalence between the massive Thirring model and the Sine-Gordon model [46], on the one hand, and the equivalence between the Sine-Gordon model and the Coulomb gas [60], on the other hand, to relate the random $X Y$ model to interacting Dirac fermions in the presence of random vector potentials. For a Gaussian distribution of the randomness with small enough strength (variance) $0<\sigma<\sigma_{\mathrm{cr}}$, Rubinstein et al. predicted a phase with algebraic decaying correlations at finite temperatures for the disordered system. Korshunov [61] has argued that this intermediate phase with quasilong range order is not stable. The source of the instability in Korshunov's analysis is very reminiscent of the special instability induced by large powers of the mass operator for our Dirac spinors. It would thus be extremely useful to understand in the context of the replica trick how to describe the special instability of non-interacting Dirac fermions in the presence of vector gauge-like disorder.

\title{
ACKNOWLEDGMENTS
}

We would like to thank B. Altshuler, H. Carruzzo, E. Fradkin, M. Janssen, M. Kardar, Y. B. Kim, P. A. Lee, and Z. Q. Wang for sharing their insights. This work was supported by NSF grants DMR-9411574 (XGW) and DMR-9400334 (CCC). CM acknowledges a fellowship from the Swiss Nationalfonds and XGW acknowledges the support from A.P. Sloan Foundation.

\section{APPENDIX A: U(1/1) SUGUWARA CONSTRUCTION}

\author{
a. Semi-direct algebra
}

We assume the formal construction [62]

$$
T(z)=\kappa_{1}(J j+\eta \bar{\eta}-\bar{\eta} \eta)(z)+\kappa_{2}(J J)(z)
$$

for the holomorphic component of the energy momentum tensor out of the holomorphic components of the currents $J, j, \eta, \bar{\eta}$ obeying a $U(1 / 1)$ Kac-Moody algebra. Above definition is meaningless until what is meant by the products of currents at the same point is specified. To this end we define the Laurent series

$$
I^{a}(z)=\sum_{n \in \mathbb{Z}} z^{-n-1} I_{n}^{a}, \quad I^{a} \in\{J, j, \eta, \bar{\eta}\} .
$$

The algebra obeyed by the normal modes $I_{n}^{a}$ of the currents $I^{a}(z)$ is

$$
\left[I_{m}^{a}, I_{n}^{b}\right]_{ \pm} \equiv k^{a b} m \delta_{m,-n}+f^{a b c} I_{m+n}^{c},
$$

where the bracket $[A, B]_{+}$is the anticommutator $\{A, B\}$ for $A, B$ two fermionic operators, and the bracket $[A, B]_{-}$ is the commutator $[A, B]$ for all other cases. According to Eq. (6.29), the only non-vanishing central extensions $k^{a b}$ and structure constants $f^{a b c}$ are

$$
\begin{aligned}
& k^{12}=k^{21}=2 k, \quad k^{22}=k_{j}, \quad k^{34}=-k^{43}=-k, \\
& f^{233}=-f^{323}=-2, \quad f^{244}=-f^{424}=+2, \quad f^{341}=f^{431}=+1 .
\end{aligned}
$$


From the algebraic point of view, $k$ and $k_{j}$ can be taken to be arbitrary complex numbers. The 12 commutators and 4 anticommutators for the generators of the $U(1 / 1)$ Kac-Moody algebra are explicitly given by

$$
\begin{aligned}
& {\left[J_{m}, J_{n}\right]=0, \quad\left[J_{m}, j_{n}\right]=2 k m \delta_{m,-n}, \quad\left[J_{m}, \eta_{n}\right]=0, \quad\left[J_{m}, \bar{\eta}_{n}\right]=0,} \\
& {\left[j_{m}, J_{n}\right]=2 k m \delta_{m,-n},\left[j_{m}, j_{n}\right]=k_{j} m \delta_{m,-n},\left[j_{m}, \eta_{n}\right]=-2 \eta_{m+n},\left[j_{m}, \bar{\eta}_{n}\right]=+2 \bar{\eta}_{m+n},} \\
& {\left[\eta_{m}, J_{n}\right]=0, \quad\left[\eta_{m}, j_{n}\right]=+2 \eta_{m+n}, \quad\left\{\eta_{m}, \eta_{n}\right\}=0, \quad\left\{\eta_{m}, \bar{\eta}_{n}\right\}=-k m \delta_{m,-n}+J_{m+n},} \\
& {\left[\bar{\eta}_{m}, J_{n}\right]=0,\left[\bar{\eta}_{m}, j_{n}\right]=-2 \bar{\eta}_{m+n},\left\{\bar{\eta}_{m}, \eta_{n}\right\}=+k m \delta_{m,-n}+J_{m+n},\left\{\bar{\eta}_{m}, \bar{\eta}_{n}\right\}=0 .}
\end{aligned}
$$

Having defined the Kac-Moody algebra $U(1 / 1)$, we introduce the convolutions $L_{m}$ of the currents through

$$
\begin{aligned}
L_{m} & =\kappa_{1} \sum_{m^{\prime} \in \mathbf{Z}}:\left(J_{m+m^{\prime}} j_{-m^{\prime}}+\eta_{m+m^{\prime}} \bar{\eta}_{-m^{\prime}}-\bar{\eta}_{m+m^{\prime}} \eta_{-m^{\prime}}\right):+\kappa_{2} \sum_{m^{\prime} \in \mathbb{Z}}: J_{m+m^{\prime}} J_{-m^{\prime}}: \\
& =\kappa_{1} \sum_{m+m^{\prime}<0}\left(J_{m+m^{\prime}} j_{-m^{\prime}}+\eta_{m+m^{\prime}} \bar{\eta}_{-m^{\prime}}-\bar{\eta}_{m+m^{\prime}} \eta_{-m^{\prime}}\right)+\kappa_{2} \sum_{m+m^{\prime}<0} J_{m+m^{\prime}} J_{-m^{\prime}} \\
& +\kappa_{1} \sum_{m+m^{\prime} \geq 0}\left(j_{-m^{\prime}} J_{m+m^{\prime}}-\bar{\eta}_{-m^{\prime}} \eta_{m+m^{\prime}}+\eta_{-m^{\prime}} \bar{\eta}_{m+m^{\prime}}\right)+\kappa_{2} \sum_{m+m^{\prime} \geq 0} J_{-m^{\prime}} J_{m+m^{\prime}} .
\end{aligned}
$$

The parameters $\kappa_{1,2}$ are complex and to be determined below. Here, the normal ordering is defined by the second line on the right-hand-side. We now show that the property

$$
\left[L_{m}, I_{n}^{a}\right]=-n I_{m+n}^{a}, \quad \forall m, n \in \mathbf{Z},
$$

holds if and only if

$$
\kappa_{1}=\frac{1}{2 k}, \quad \kappa_{2}=\frac{4-k_{j}}{4 k} \kappa_{1}
$$

Notice that it follows from Eq. (A7) that all $L_{m}$ are left invariant by the generators $I_{0}^{a}, a=1, \cdots, 4$.

PROOF: Let $m, n$ be arbitrary integers. Decompose $L_{m}$ into three contributions:

$$
\begin{aligned}
L_{m}^{1} & =\kappa_{1} \sum_{m^{\prime} \in \mathbb{Z}}: J_{m+m^{\prime}} j_{-m^{\prime}}:, \\
L_{m}^{2} & =\kappa_{1} \sum_{m^{\prime} \in \mathbb{Z}}:\left(\eta_{m+m^{\prime}} \bar{\eta}_{-m^{\prime}}-\bar{\eta}_{m+m^{\prime}} \eta_{-m^{\prime}}\right):, \\
L_{m}^{3} & =\kappa_{2} \sum_{m^{\prime} \in \mathbb{Z}}: J_{m+m^{\prime}} J_{-m^{\prime}}: .
\end{aligned}
$$

The case $a=1$ is straightforward. It yields a constraint on $\kappa_{1}$. Indeed, due to Eq. (A5a), $\left[L_{m}, J_{n}\right]=\left[L_{m}^{1}, J_{n}\right]$. Now,

$$
\begin{aligned}
{\left[L_{m}^{1}, J_{n}\right] } & =\kappa_{1} \sum_{m+m^{\prime}<0}\left(J_{m+m^{\prime}}\left[j_{-m^{\prime}}, J_{n}\right]+\left[J_{m+m^{\prime}}, J_{n}\right] j_{-m^{\prime}}\right) \\
& +\kappa_{1} \sum_{m+m^{\prime} \geq 0}\left(j_{-m^{\prime}}\left[J_{m+m^{\prime}}, J_{n}\right]+\left[j_{-m^{\prime}}, J_{n}\right] J_{m+m^{\prime}}\right) \\
& =\kappa_{1} \sum_{m+m^{\prime}<0} J_{m+m^{\prime}} 2 k\left(-m^{\prime}\right) \delta_{-m^{\prime},-n}+\kappa_{1} \sum_{m+m^{\prime} \geq 0} 2 k\left(-m^{\prime}\right) \delta_{-m^{\prime},-n} J_{m+m^{\prime}} \\
& =-n 2 k \kappa_{1} J_{m+n} .
\end{aligned}
$$

The case $a=2$ is more involved. It yields a constraint on $\kappa_{1}$ and $\kappa_{2}$. The first contribution is

$$
\begin{aligned}
{\left[L_{m}^{1}, j_{n}\right] } & =\kappa_{1} \sum_{m+m^{\prime}<0}\left(J_{m+m^{\prime}}\left[j_{-m^{\prime}}, j_{n}\right]+\left[J_{m+m^{\prime}}, j_{n}\right] j_{-m^{\prime}}\right) \\
& +\kappa_{1} \sum_{m+m^{\prime} \geq 0}\left(j_{-m^{\prime}}\left[J_{m+m^{\prime}}, j_{n}\right]+\left[j_{-m^{\prime}}, j_{n}\right] J_{m+m^{\prime}}\right) \\
& =\kappa_{1} \sum_{m+m^{\prime}<0}\left(J_{m+m^{\prime}} k_{j}\left(-m^{\prime}\right) \delta_{-m^{\prime},-n}+2 k\left(m+m^{\prime}\right) \delta_{m+m^{\prime},-n} j_{-m^{\prime}}\right) \\
& +\kappa_{1} \sum_{m+m^{\prime} \geq 0}\left(j_{-m^{\prime}} 2 k\left(m+m^{\prime}\right) \delta_{m+m^{\prime},-n}+k_{j}\left(-m^{\prime}\right) \delta_{-m^{\prime},-n} J_{m+m^{\prime}}\right) \\
& =-n 2 k \kappa_{1} j_{m+n}-n k_{j} \kappa_{1} J_{m+n} .
\end{aligned}
$$


The second contribution is

$$
\begin{aligned}
& {\left[L_{m}^{2}, j_{n}\right]=} \\
& +\kappa_{1} \sum_{m+m^{\prime}<0}\left(\eta_{m+m^{\prime}}\left[\bar{\eta}_{-m^{\prime}}, j_{n}\right]+\left[\eta_{m+m^{\prime}}, j_{n}\right] \bar{\eta}_{-m^{\prime}}-\bar{\eta}_{m+m^{\prime}}\left[\eta_{-m^{\prime}}, j_{n}\right]-\left[\bar{\eta}_{m+m^{\prime}}, j_{n}\right] \eta_{-m^{\prime}}\right) \\
& -\kappa_{1} \sum_{m+m^{\prime} \geq 0}\left(\bar{\eta}_{-m^{\prime}}\left[\eta_{m+m^{\prime}}, j_{n}\right]+\left[\bar{\eta}_{-m^{\prime}}, j_{n}\right] \eta_{m+m^{\prime}}-\eta_{-m^{\prime}}\left[\bar{\eta}_{m+m^{\prime}}, j_{n}\right]-\left[\eta_{-m^{\prime}}, j_{n}\right] \bar{\eta}_{m+m^{\prime}}\right)= \\
& -2 \kappa_{1}\left[\sum_{m+m^{\prime}<0}\left(\eta_{m+m^{\prime}} \bar{\eta}_{n-m^{\prime}}+\bar{\eta}_{m+m^{\prime}} \eta_{n-m^{\prime}}\right)-\sum_{m+m^{\prime}<0}\left(\eta_{m+n+m^{\prime}} \bar{\eta}_{-m^{\prime}}+\bar{\eta}_{m+n+m^{\prime}} \eta_{-m^{\prime}}\right)\right] \\
& +2 \kappa_{1}\left[\sum_{m+m^{\prime} \geq 0}\left(\bar{\eta}_{n-m^{\prime}} \eta_{m+m^{\prime}}+\eta_{n-m^{\prime}} \bar{\eta}_{m+m^{\prime}}\right)-\sum_{m+m^{\prime} \geq 0}\left(\bar{\eta}_{-m^{\prime}} \eta_{m+n+m^{\prime}}+\eta_{-m^{\prime}} \bar{\eta}_{m+n+m^{\prime}}\right)\right] \text {. }
\end{aligned}
$$

It is very important to account for the fermionic sign in the normal ordering of $L_{m}^{2}$. This allows us to reduce the infinite sum to a finite sum. To see this, introduce the integer

$$
I=\min \{-m,-m+n\},
$$

and change variables in the second sums in the square brackets:

$$
\begin{aligned}
& {\left[L_{m}^{2}, j_{n}\right]=} \\
& -2 \kappa_{1}\left[\sum_{m^{\prime}<-m}\left(\eta_{m+m^{\prime}} \bar{\eta}_{n-m^{\prime}}+\bar{\eta}_{m+m^{\prime}} \eta_{n-m^{\prime}}\right)-\sum_{m^{\prime \prime}<-m+n}\left(\eta_{m+m^{\prime \prime}} \bar{\eta}_{n-m^{\prime \prime}}+\bar{\eta}_{m+m^{\prime \prime}} \eta_{n-m^{\prime \prime}}\right)\right] \\
& +2 \kappa_{1}\left[\sum_{m^{\prime} \geq-m}\left(\bar{\eta}_{n-m^{\prime}} \eta_{m+m^{\prime}}+\eta_{n-m^{\prime}} \bar{\eta}_{m+m^{\prime}}\right)-\sum_{m^{\prime \prime} \geq-m+n}\left(\bar{\eta}_{n-m^{\prime \prime}} \eta_{m+m^{\prime \prime}}+\eta_{n-m^{\prime}} \bar{\eta}_{m+m^{\prime \prime}}\right)\right]= \\
& 2 \kappa_{1} \frac{n}{|n|}\left[\sum_{m^{\prime}=I}^{I+|n|-1}\left(\eta_{m+m^{\prime}} \bar{\eta}_{n-m^{\prime}}+\bar{\eta}_{m+m^{\prime}} \eta_{n-m^{\prime}}\right)+\sum_{m^{\prime}=I}^{I+|n|-1}\left(\bar{\eta}_{n-m^{\prime}} \eta_{m+m^{\prime}}+\eta_{n-m^{\prime}} \bar{\eta}_{m+m^{\prime}}\right)\right] .
\end{aligned}
$$

We are thus left with the finite sum

$$
\begin{aligned}
{\left[L_{m}^{2}, j_{n}\right] } & =2 \kappa_{1} \frac{n}{|n|} \sum_{m^{\prime}=I}^{I+|n|-1}\left(\left\{\eta_{m+m^{\prime}}, \bar{\eta}_{n-m^{\prime}}\right\}+\left\{\bar{\eta}_{m+m^{\prime}}, \eta_{n-m^{\prime}}\right\}\right) \\
& =2 \kappa_{1} \frac{n}{|n|} \sum_{m^{\prime}=I}^{I+|n|-1}\left(-k\left(m+m^{\prime}\right) \delta_{m+m^{\prime},-n+m^{\prime}}+J_{m+n}\right. \\
& \left.+k\left(m+m^{\prime}\right) \delta_{m+m^{\prime},-n+m^{\prime}}+J_{m+n}\right) \\
& =+n 4 \kappa_{1} J_{m+n} .
\end{aligned}
$$

Finally, the third contribution is

$$
\begin{aligned}
{\left[L_{m}^{3}, j_{n}\right] } & =\kappa_{2} \sum_{m+m^{\prime}<0}\left(J_{m+m^{\prime}}\left[J_{-m^{\prime}}, j_{n}\right]+\left[J_{m+m^{\prime}}, j_{n}\right] J_{-m^{\prime}}\right) \\
& +\kappa_{2} \sum_{m+m^{\prime} \geq 0}\left(J_{-m^{\prime}}\left[J_{m+m^{\prime}}, j_{n}\right]+\left[J_{-m^{\prime}}, j_{n}\right] J_{m+m^{\prime}}\right) \\
& =2 k \kappa_{2} \sum_{m+m^{\prime}<0}\left(J_{m+m^{\prime}}\left(-m^{\prime}\right) \delta_{-m^{\prime},-n}+\left(m+m^{\prime}\right) \delta_{m+m^{\prime},-n} J_{-m^{\prime}}\right) \\
& +\kappa_{2} \sum_{m+m^{\prime} \geq 0}\left(J_{-m^{\prime}}\left(m+m^{\prime}\right) \delta_{m+m^{\prime},-n}+\left(-m^{\prime}\right) \delta_{-m^{\prime},-n} J_{m+m^{\prime}}\right) \\
& =-n 4 k \kappa_{2} J_{m+n} .
\end{aligned}
$$


Collecting all three contributions yield

$$
\left[L_{m}, j_{n}\right]=-n 2 k \kappa_{1} j_{m+n}-n\left(k_{j} \kappa_{1}-4 \kappa_{1}+4 k \kappa_{2}\right) J_{m+n} .
$$

Without loss of generality, we only consider the case $a=3$, the case $a=4$ being similar. We show that $\kappa_{1}$ and $\kappa_{2}$ are not overdetermined. We only need the commutators of $\eta_{n}$ with $L_{m}^{1}$ and $L_{m}^{2}$ since $J_{m}$ commutes with $\eta_{n}$. The first commutator is

$$
\begin{aligned}
{\left[L_{m}^{1}, \eta_{n}\right] } & =\kappa_{1} \sum_{m+m^{\prime}<0}\left(J_{m+m^{\prime}}\left[j_{-m^{\prime}}, \eta_{n}\right]+\left[J_{m+m^{\prime}}, \eta_{n}\right] j_{-m^{\prime}}\right) \\
& +\kappa_{1} \sum_{m+m^{\prime} \geq 0}\left(j_{-m^{\prime}}\left[J_{m+m^{\prime}}, \eta_{n}\right]+\left[j_{-m^{\prime}}, \eta_{n}\right] J_{m+m^{\prime}}\right) \\
& =-2 \kappa_{1}\left[\sum_{m+m^{\prime}<0} J_{m+m^{\prime}} \eta_{n-m^{\prime}}+\sum_{m+m^{\prime} \geq 0} \eta_{n-m^{\prime}} J_{m+m^{\prime}}\right] \\
& =-2 \kappa_{1} \sum_{m^{\prime} \in \mathbb{Z}} J_{m+m^{\prime}} \eta_{n-m^{\prime}} .
\end{aligned}
$$

The second commutator is

$$
\begin{aligned}
& {\left[L_{m}^{2}, \eta_{n}\right]=} \\
& +\kappa_{1} \sum_{m+m^{\prime}<0}\left(\eta_{m+m^{\prime}}\left\{\bar{\eta}_{-m^{\prime}}, \eta_{n}\right\}-\left\{\eta_{m+m^{\prime}}, \eta_{n}\right\} \bar{\eta}_{-m^{\prime}}-\bar{\eta}_{m+m^{\prime}}\left\{\eta_{-m^{\prime}}, \eta_{n}\right\}+\left\{\bar{\eta}_{m+m^{\prime}}, \eta_{n}\right\} \eta_{-m^{\prime}}\right) \\
& -\kappa_{1} \sum_{m+m^{\prime} \geq 0}\left(\bar{\eta}_{-m^{\prime}}\left\{\eta_{m+m^{\prime}}, \eta_{n}\right\}-\left\{\bar{\eta}_{-m^{\prime}}, \eta_{n}\right\} \eta_{m+m^{\prime}}-\eta_{-m^{\prime}}\left\{\bar{\eta}_{m+m^{\prime}}, \eta_{n}\right\}+\left\{\eta_{-m^{\prime}}, \eta_{n}\right\} \bar{\eta}_{m+m^{\prime}}\right)= \\
& +\kappa_{1} \sum_{m+m^{\prime}<0}\left(\eta_{m+m^{\prime}} k\left(-m^{\prime}\right) \delta_{-m^{\prime},-n}+\eta_{m+m^{\prime}} J_{n-m^{\prime}}+k\left(m+m^{\prime}\right) \delta_{m+m^{\prime},-n} \eta_{-m^{\prime}}+J_{m+n+m^{\prime}} \eta_{-m^{\prime}}\right) \\
& +\kappa_{1} \sum_{m+m^{\prime} \geq 0}\left(k\left(-m^{\prime}\right) \delta_{-m^{\prime},-n} \eta_{m+m^{\prime}}+J_{n-m^{\prime}} \eta_{m+m^{\prime}}+\eta_{-m^{\prime}} k\left(m+m^{\prime}\right) \delta_{m+m^{\prime},-n}+\eta_{-m^{\prime}} J_{m+n}\right)= \\
& -n 2 k \kappa_{1} \eta_{m+n}+2 \kappa_{1} \sum_{m^{\prime} \in \mathbf{Z}} J_{m+m^{\prime}} \eta_{n-m^{\prime}} .
\end{aligned}
$$

Collecting all terms, we find

$$
\left[L_{m}, \eta_{n}\right]=-n 2 k \kappa_{1} \eta_{m+n}
$$

This concludes the proof.

\section{b. Virasoro algebra}

We now show that the set $\left\{L_{m}\right\}_{m \in \mathbf{Z}}$ generates a Virasoro algebra with vanishing central charge, provided $\kappa_{1}$ and $\kappa_{2}$ are chosen as in Eq. (A8):

$$
\left[L_{m}, L_{n}\right]=(m-n) L_{m+n} .
$$

PROOF: Let $m, n$ be arbitrary integers. The first commutator to be considered is

$$
\begin{aligned}
{\left[L_{m}, L_{n}^{1}\right] } & =\kappa_{1} \sum_{n+n^{\prime}<0}\left[L_{m}, J_{n+n^{\prime}} j_{-n^{\prime}}\right]+\kappa_{1} \sum_{n+n^{\prime} \geq 0}\left[L_{m}, j_{-n^{\prime}} J_{n+n^{\prime}}\right] \\
& =\kappa_{1} \sum_{n+n^{\prime}<0} n^{\prime} J_{n+n^{\prime}} j_{m-n^{\prime}}-\kappa_{1} \sum_{n+n^{\prime}<0}\left(n+n^{\prime}\right) J_{m+n+n^{\prime}} j_{-n^{\prime}} \\
& +\kappa_{1} \sum_{n+n^{\prime} \geq 0} n^{\prime} j_{m-n^{\prime}} J_{n+n^{\prime}}-\kappa_{1} \sum_{n+n^{\prime} \geq 0}\left(n+n^{\prime}\right) j_{-n^{\prime}} J_{m+n+n^{\prime}} .
\end{aligned}
$$


With the help of the change of variables $n^{\prime}=m+n^{\prime \prime}$ in the first summation on the last two lines and with the introduction of

$$
I=\min \{-n,-n-m\}
$$

we can rewrite the right-hand-side as

$$
\begin{aligned}
{\left[L_{m}, L_{n}^{1}\right] } & =\kappa_{1} \sum_{n+m+n^{\prime \prime}<0}\left(m+n^{\prime \prime}\right) J_{n+m+n^{\prime \prime}} j_{-n^{\prime \prime}}-\kappa_{1} \sum_{m+n+n^{\prime}<0}\left(n+n^{\prime}\right) J_{m+n+n^{\prime}} j_{-n^{\prime}} \\
& +\kappa_{1} \sum_{n+m+n^{\prime \prime} \geq 0}\left(m+n^{\prime \prime}\right) j_{-n^{\prime \prime}} J_{n+m+n^{\prime \prime}}-\kappa_{1} \sum_{m+n+n^{\prime} \geq 0}\left(n+n^{\prime}\right) j_{-n^{\prime}} J_{m+n+n^{\prime}} \\
& -\kappa_{1} \frac{m}{|m|} \sum_{n^{\prime}=I}^{I+|m|-1}\left(n+n^{\prime}\right) J_{m+n+n^{\prime}} j_{-n^{\prime}}+\kappa_{1} \frac{m}{|m|} \sum_{n^{\prime}=I}^{I+|m|-1}\left(n+n^{\prime}\right) j_{-n^{\prime}} J_{m+n+n^{\prime}} \\
& =(m-n) L_{m+n}^{1}-\kappa_{1} \frac{m}{|m|} \sum_{n^{\prime}=I}^{I+|m|-1}\left(n+n^{\prime}\right)\left[J_{m+n+n^{\prime}}, j_{-n^{\prime}}\right] \\
& =(m-n) L_{m+n}^{1}-2 k \kappa_{1} \frac{m}{|m|} \sum_{n^{\prime}=I}^{I+|m|-1}\left(n+n^{\prime}\right)\left(m+n+n^{\prime}\right) \delta_{m+n+n^{\prime}, n^{\prime}} \\
& =(m-n) L_{m+n}^{1}-2 k \kappa_{1} \frac{m}{|m|} \delta_{m,-n} \sum_{n^{\prime}=I}^{I+|m|-1}\left(n+n^{\prime}\right) n^{\prime} .
\end{aligned}
$$

Because the commutators of $J_{p}$ with $J_{q}$ vanishes for any integers $p$ and $q$, one immediately obtains from $\left[L_{m}, L_{n}^{1}\right]$ that

$$
\left[L_{m}, L_{n}^{3}\right]=(m-n) L_{m+n}^{3} .
$$

The proof that the Virasoro central charge vanishes is then established by showing that

$$
\left[L_{m}, L_{n}^{2}\right]=(m-n) L_{m+n}^{2}+2 k \kappa_{1} \frac{m}{|m|} \delta_{m,-n} \sum_{n^{\prime}=I}^{I+|m|-1}\left(n+n^{\prime}\right) n^{\prime} .
$$

To show this, start from

$$
\begin{aligned}
& {\left[L_{m}, L_{n}^{2}\right]=\kappa_{1} \sum_{n+n^{\prime}<0}\left[L_{m},\left(\eta_{n+n^{\prime}} \bar{\eta}_{-n^{\prime}}-\bar{\eta}_{n+n^{\prime}} \eta_{-n^{\prime}}\right)\right]-\kappa_{1} \sum_{n+n^{\prime} \geq 0}\left[L_{m},\left(\bar{\eta}_{-n^{\prime}} \eta_{n+n^{\prime}}-\eta_{-n^{\prime}} \bar{\eta}_{n+n^{\prime}}\right)\right]=} \\
& \kappa_{1} \sum_{n+n^{\prime}<0} n^{\prime}\left(\eta_{n+n^{\prime}} \bar{\eta}_{m-n^{\prime}}-\bar{\eta}_{n+n^{\prime}} \eta_{m-n^{\prime}}\right)-\kappa_{1} \sum_{n+n^{\prime}<0}\left(n+n^{\prime}\right)\left(\eta_{m+n+n^{\prime}} \bar{\eta}_{-n^{\prime}}-\bar{\eta}_{m+n+n^{\prime}} \eta_{-n^{\prime}}\right) \\
& -\kappa_{1} \sum_{n+n^{\prime} \geq 0} n^{\prime}\left(\bar{\eta}_{m-n^{\prime}} \eta_{n+n^{\prime}}-\eta_{m-n^{\prime}} \bar{\eta}_{n+n^{\prime}}\right)+\kappa_{1} \sum_{n+n^{\prime} \geq 0}\left(n+n^{\prime}\right)\left(\bar{\eta}_{-n^{\prime}} \eta_{m+n+n^{\prime}}-\eta_{-n^{\prime}} \bar{\eta}_{m+n+n^{\prime}}\right) .
\end{aligned}
$$

With the help of the substitution $n^{\prime}=m+n^{\prime \prime}$ in the first summation on the last two lines, we can again extract $(m-n) L_{m+n}^{2}$. The reminder is a finite sum over anticommutators due to the fermionic normal ordering:

$$
\left[L_{m}, L_{n}^{2}\right]=(m-n) L_{m+n}^{2}-\kappa_{1} \frac{m}{|m|} \sum_{n^{\prime}=I}^{I+|m|-1}\left(n+n^{\prime}\right)\left(\left\{\eta_{m+n+n^{\prime}}, \bar{\eta}_{-n^{\prime}}\right\}-\left\{\bar{\eta}_{m+n+n^{\prime}}, \eta_{-n^{\prime}}\right\}\right) .
$$

The term $J_{m+n}$ cancels from the difference of the two anticommutators and we are left with twice the contribution from the central charge $k$ to the OPE between $\eta$ and $\bar{\eta}$ as given in Eq. (A26). This concludes the proof.

\section{APPENDIX B: LAGRANGIAN REALIZATION OF $U(1 / 1) \times U(1 / 1)$ CURRENT ALGEBRA}

In this appendix, we show that the $U(1 / 1) \times U(1 / 1)$ Kac-Moody algebra of Eq. (A4) or Eq. (6.29) with real central charges $\left(k, k_{j}\right)$ is associative. To prove associativity, it is sufficient to construct a Lagrangian realization of a free 
field theory, whose currents obey the algebra of Eq. (6.29) with $\left(k, k_{j}\right)=\left(1, k_{j}\right), k_{j} \geq 0$. Associativity for arbitrary values of $\left(k, k_{j}\right)$, where $k \neq 0$, then follows on purely algebraic grounds by independent rescalings of the currents $(J, j, \eta, \bar{\eta})$. We have already seen a Lagrangian realization of the current algebra with central charges $\left(k, k_{j}\right)=(1,0)$ in Eq. (6.1). We now construct a Lagrangian realization of $U(1 / 1) \times U(1 / 1)$ Kac-Moody algebra with central charges $\left(k, k_{j}\right)=\left(1, k_{j}\right), k_{j}>0$. (For a similar trick, see Ref. [63]) Consider the partition function

$$
\begin{aligned}
& \mathcal{Z}_{0}^{\left(1, k_{j}\right)}=\int \mathcal{D}[\phi] \int \mathcal{D}\left[\psi_{ \pm}^{\dagger}, \psi_{ \pm}\right] \int \mathcal{D}\left[\varphi_{ \pm}^{\dagger}, \varphi_{ \pm}\right] e^{-\int_{-\infty}^{+\infty} d x d \tau\left(\mathcal{L}_{0}+\mathcal{L}_{k_{j}}\right)} \\
& \mathcal{L}_{0}=\frac{1}{\pi}\left(\psi_{+}^{\dagger} \partial_{\bar{z}} \psi_{+}+\varphi_{+}^{\dagger} \partial_{\bar{z}} \varphi_{+}+\psi_{-}^{\dagger} \partial_{z} \psi_{-}+\varphi_{-}^{\dagger} \partial_{z} \varphi_{-}\right) \\
& \mathcal{L}_{k_{j}}=\frac{1}{2} \sqrt{\frac{1}{8 \pi}}\left(\partial_{z} \phi\right)\left(\partial_{\bar{z}} \phi\right) .
\end{aligned}
$$

Define the holomorphic and antiholomorphic components of the free real scalar field $\phi(z, \bar{z})$ by (compare with Eq. (3.22)

$$
\phi(z, \bar{z})=\phi(z)+\bar{\phi}(\bar{z}), \quad\langle\phi(z) \phi(0)\rangle=-2 \ln z, \quad\langle\bar{\phi}(\bar{z}) \bar{\phi}(0)\rangle=-2 \ln \bar{z}, \quad\langle\phi(z) \bar{\phi}(0)\rangle=0 .
$$

The real scalar field $\phi$ is taken to commute with all spinors. Without loss of generality, we only consider the holomorphic sector. Define the holomorphic component of the currents

$$
\begin{aligned}
& J(z)=\left(\psi_{+}^{\dagger} \psi_{+}\right)(z)+\left(\varphi_{+}^{\dagger} \varphi_{+}\right)(z), \\
& j(z)=\left(\psi_{+}^{\dagger} \psi_{+}\right)(z)-\left(\varphi_{+}^{\dagger} \varphi_{+}\right)(z)+\mathrm{i} \sqrt{\frac{k_{j}}{2}} \partial_{z} \phi(z), \\
& \eta(z)=\left(\varphi_{+}^{\dagger} \psi_{+}\right)(z), \\
& \bar{\eta}(z)=\left(\psi_{+}^{\dagger} \varphi_{+}\right)(z) .
\end{aligned}
$$

The structure constants $\left\{f_{a b c}\right\}$ for the OPE of these currents are those of $U(1 / 1)$, since $\phi$ commutes with the spinors. By assuming that currents do not acquire vacuum expectation values, the only change in the algebra takes place in the OPE $j(z) j(0)=\frac{k_{j}}{z^{2}}$. Note the similarity between Eq. (B1) and the effective critical theory defined by $S_{\mathrm{cr}}^{(1,0)}$ in Eq. (3.25).

[1] B. Huckestein, cond-mat/9501106, and references therein.

[2] S.A. Trugman, Phys. Rev. B 27 (1983) 7539.

[3] J.T. Chalker and P. D. Coddington, J. Phys. C 21 (1988) 2665.

[4] H. Levine, S.B. Libby, and A.M.M. Pruisken, Phys. Rev. Lett. 20 (1983) 1915; A.M.M. Pruisken, Nucl. Phys. B 235 (1984) 277; H. Levine, S.B. Libby, and A.M.M. Pruisken, Nucl. Phys. B 240 (1984) 30, 49, 71; D.E. Khmel'nitskii, JETP Lett. 38 (1983) 552.

[5] I. Affleck, Nucl. Phys. B 257 (1985) 397; I. Affleck, Nucl. Phys. B 265 (1986) 409; I. Affleck, Nucl. Phys. B 305 (1988) 582.

[6] M.R. Zirnbauer, Ann. Physik 3 (1994) 513.

[7] D.-H. Lee and Z.Q. Wang, cond-mat/9503022.

[8] F. Wegner, Z. Phys. B 25 (1976) 327.

[9] E.P. Abrahams, P.W. Anderson, D.C. Licciardello, and V. Ramakrishnan, Phys. Rev. Lett. 42 (1979) 673.

[10] M. Janssen, Int. J. of Mod. Phys. B 8 (1994) 943.

[11] B. Duplantier and A.W.W. Ludwig, Phys. Rev. Lett. 66 (1991) 247.

[12] F. Wegner, Z. Physik B 36 (1980) 209.

[13] F. Wegner in: "Localization and Metal Insulator Transitions", H. Fritzsche and D. Adler (eds.), Institute for Amorphous Studies Series, Plenum (New-York) 1985.

[14] F. Wegner, Nucl. Phys. B 280 (1987) 210.

[15] C. Castellani and L. Peliti, J. Phys. A 19 (1986) L429.

[16] X.-G. Wen and Y.-S. Wu, Phys. Rev. Lett. 70 (1993) 1501. 
[17] W. Chen, M. Fisher, and Y.-S. Wu, Phys. Rev. B 48 (1993) 13749.

[18] A.W.W. Ludwig, Nucl. Phys. B 285 (1987) 97.

[19] A.W.W. Ludwig, Nucl. Phys. B 330 (1990) 639.

[20] A. Ludwig, M. Fisher, R. Shankar and G. Grinstein, Phys. Rev. B 50 (1994) 7526.

[21] E. Fradkin, Phys. Rev. B 33 (1986) 3257.

[22] G.W. Semenoff, Phys. Rev. Lett. 53 (1984) 2449.

[23] M.P.A. Fisher and E. Fradkin, Nucl. Phys. B 251 (1985) 457.

[24] P.A. Lee, Phys. Rev. Lett. 71 (1993) 1887.

[25] A.A. Nersesyan, A.M. Tsvelik, and F. Wenger, Phys. Rev. Lett. 72 (1994) 2628; A.A. Nersesyan, A.M. Tsvelik, and F. Wenger, Nucl. Phys. B 438 (1995) 561.

[26] V.S. Dotsenko and V. Dotsenko, Adv. Phys. 32 (1983) 129.

[27] R. Shankar, Phys. Rev. Lett. 58 (1987) 2466.

[28] K.B. Efetov, Adv. in Phys. 32 (1983) 53.

[29] D. Bernard, Nucl. Phys. B 441 (1995) 471.

[30] R.M. Gade and F. Wegner, Nucl. Phys. B 360 (1991) 213.

[31] S. Hikami, M. Shirai, and F. Wegner, Nucl. Phys. B 408 (1993) 415.

[32] F. Wegner, Z. Phys. 51 (1983) 279.

[33] E. Brézin, D. J. Gross, and C. Itzykson, Nucl. Phys. B 235 (1984) 24.

[34] C. Chamon, C. Mudry, and X.-G. Wen, cond-mat/9501066.

[35] E. Brézin, J. Zinn-Justin and J.C. Le Guillou, Phys. Rev. B 14 (1976) 4976.

[36] F. Wegner, Z. Phys. B 78 (1990) 33.

[37] G.E. Castilla and S. Chakravarty, Phys. Rev. Lett. 71 (1993) 384.

[38] C. Chamon, C. Mudry, and X.-G. Wen, work in progress.

[39] M.E. Cates and J.M. Deutsch, Phys. Rev. A 35 (1987) 4907.

[40] A.A. Belavin, A.M. Polyakov, and A.B. Zamolodchikov, Nucl. Phys. B 241 (1984) 333.

[41] A.J. McKane and M. Stone, Ann. of Phys. 131 (1981) 36, and references therein.

[42] K. Furuya, R.E. Gamboa Saravi, and F.A. Schaposnik, Nucl. Phys. B 208 (1982) 159.

[43] I.V. Belvedere, J.A. Swieca, K.D. Rothe, and B. Schroer, Nucl. Phys. B 153 (1979) 112.

[44] K. Fujikawa, Phys. Rev. Lett. 42 (1979) 1195; Phys. Rev. D 21 (1980) 2848.

[45] R. Roskies and F. Schaposnik, Phys. Rev. D 23 (1981) 558.

[46] For a review, see "Non-perturbative methods in 2 dimensional quantum field theory", by E. Abdalla, M.C.B. Abdalla, and K. Rothe, (Singapore, 1991).

[47] A.M. Tsvelik, Phys. Rev. B 51 (1995) 9449.

[48] See for example, A.M. Polyakov in Fields, Strings and Critical Phenomena, edited by E. Brézin and J. Zinn-Justin Les Houches, Session XLIX, 1988 (Elsevier Science, 1989).

[49] A.M. Polyakov and P.M. Wiegmann, Phys. Lett. 131B (1983) 121.

[50] A. M. Polyakov and P. M. Wiegmann, Phys. Lett. 141B (1984) 223.

[51] V.G. Knizhnik and A.B. Zamolodchikov, Nucl. Phys. B 247 (1984) 83.

[52] D. Karabali and H. Schnitzer, Nucl. Phys. B 329 (1990) 649; Y. Tanii, Modern Phys. Lett. A 5 (1990) 927; C. Mudry and E. Fradkin, Phys. Rev. B 50 (1994) 11409.

[53] A.M. Perelomov and V.S. Popov, Sov. J. Nucl. Phys. 3 (1966) 676.

[54] D.J. Thouless, Phys. Rep. 13 (1974) 93, and references therein.

[55] B. Fourcade, and A.-M.S. Tremblay, Phys. Rev. B 36 (1987) 2352.

[56] See for example, I. Affleck, in Fields, Strings and Critical Phenomena, edited by E. Brézin and J. Zinn-Justin Les Houches, Session XLIX, 1988 (Elsevier Science, 1989).

[57] K.G. Wilson, Phys. Rev. 179 (1969) 1499; L.P. Kadanoff, Phys. Rev. Lett. 23 (1969) 1430.

[58] See for example, P. Ginsparg, in Fields, Strings and Critical Phenomena, edited by E. Brézin and J. Zinn-Justin Les Houches, Session XLIX, 1988 (Elsevier Science, 1989).

[59] M. Rubinstein, B. Shraiman, and D. R. Nelson, Phys. Rev. B 27 (1982) 1800.

[60] For a review, see "Quantum Field Theory and Critical Phenomena" by J. Zinn-Justin (Oxford 1989).

[61] S. E. Korshunov, Phys. Rev. B 48 (1993) 1124.

[62] H. Sugawara, Phys. Rev. 170 (1968) 1659; C. Sommerfield, ibid 176 (1968) 2019; S. Coleman, D. Gross and R. Jackiw, ibid 180 (1969) 1359.

[63] A.B. Zamolodchikov and V.A. Fateev, Sov. Phys. JETP 62 (1984) 215.

TABLE I. $U(1 / 1) \times U(1 / 1)$ charges for the generators $J_{z}, j_{z}, J_{\bar{z}}, j_{\bar{z}}$

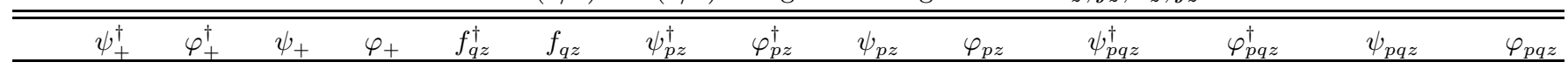




\begin{tabular}{|c|c|c|c|c|c|c|c|c|c|c|c|c|c|c|}
\hline$\underline{J_{z}}$ & 1 & 1 & -1 & -1 & 0 & 0 & $p$ & $p$ & $-p$ & $-p$ & $p$ & $p$ & $-p$ & $-p$ \\
\hline$j_{\bar{z}}$ & 0 & 0 & 0 & 0 & 0 & 0 & 0 & 0 & 0 & 0 & 0 & 0 & 0 & 0 \\
\hline
\end{tabular}

